\title{
Japanese Society for Cancer of the Colon and Rectum (JSCCR) guidelines 2019 for the treatment of colorectal cancer
}

\author{
Yojiro Hashiguchi ${ }^{1} \oplus \cdot$ Kei Muro $^{2} \cdot$ Yutaka Saito $^{3} \cdot$ Yoshinori Ito $^{4} \cdot$ Yoichi Ajioka $^{5} \cdot$ Tetsuya Hamaguchi $^{6}$. \\ Kiyoshi Hasegawa ${ }^{7} \cdot$ Kinichi Hotta $^{8} \cdot$ Hideyuki Ishida $^{9} \cdot$ Megumi Ishiguro $^{10} \cdot$ Soichiro Ishihara $^{11}$. \\ Yukihide Kanemitsu ${ }^{12}$. Yusuke Kinugasa ${ }^{13} \cdot$ Keiko Murofushi $^{14} \cdot$ Takako Eguchi Nakajima $^{15}$. Shiro Oka ${ }^{16}$. \\ Toshiaki Tanaka ${ }^{11}$ • Hiroya Taniguchi ${ }^{17}$. Akihito Tsuji ${ }^{18} \cdot K^{2}$ eisuke Uehara ${ }^{19} \cdot$ Hideki Ueno $^{20}$. Takeharu Yamanaka ${ }^{21}$. \\ Kentaro Yamazaki ${ }^{22} \cdot$ Masahiro Yoshida $^{23} \cdot$ Takayuki Yoshino $^{17} \cdot$ Michio Itabashi $^{24} \cdot$ Kentaro Sakamaki $^{25} \cdot \mathrm{Keiji} \mathrm{Sano}^{1}$. \\ Yasuhiro Shimada ${ }^{26} \cdot$ Shinji Tanaka $^{27} \cdot$ Hiroyuki Uetake $^{28} \cdot$ Shigeki Yamaguchi $^{29} \cdot$ Naohiko Yamaguchi $^{30}$. \\ Hirotoshi Kobayashi $^{31} \cdot$ Keiji Matsuda $^{1} \cdot K_{\text {Kenjiro Kotake }}{ }^{32} \cdot$ Kenichi Sugihara $^{33}$ - Japanese Society for Cancer of the \\ Colon and Rectum
}

Received: 23 May 2019 / Accepted: 29 May 2019 / Published online: 15 June 2019

(c) The Author(s) 2019

\begin{abstract}
The number of deaths from colorectal cancer in Japan continues to increase. Colorectal cancer deaths exceeded 50,000 in 2016. In the 2019 edition, revision of all aspects of treatments was performed, with corrections and additions made based on knowledge acquired since the 2016 version (drug therapy) and the 2014 version (other treatments). The Japanese Society for Cancer of the Colon and Rectum guidelines 2019 for the treatment of colorectal cancer (JSCCR guidelines 2019) have been prepared to show standard treatment strategies for colorectal cancer, to eliminate disparities among institutions in terms of treatment, to eliminate unnecessary treatment and insufficient treatment and to deepen mutual understanding between healthcare professionals and patients by making these guidelines available to the general public. These guidelines have been prepared by consensuses reached by the JSCCR Guideline Committee, based on a careful review of the evidence retrieved by literature searches and in view of the medical health insurance system and actual clinical practice settings in Japan. Therefore, these guidelines can be used as a tool for treating colorectal cancer in actual clinical practice settings. More specifically, they can be used as a guide to obtaining informed consent from patients and choosing the method of treatment for each patient. Controversial issues were selected as clinical questions, and recommendations were made. Each recommendation is accompanied by a classification of the evidence and a classification of recommendation categories based on the consensus reached by the Guideline Committee members. Here, we present the English version of the JSCCR guidelines 2019.
\end{abstract}

Keywords Colorectal cancer $\cdot$ Guideline $\cdot$ Surgery $\cdot$ Chemotherapy $\cdot$ Endoscopy $\cdot$ Radiotherapy

Introduction

\section{Guideline objectives}

According to the Vital Statistics of Japan, the number of deaths from colorectal cancer in Japan has continued to increase. In 2016, the number of deaths from colorectal cancer exceeded 50,000. Many new treatment methods have been developed and their use in combination with advances

Yojiro Hashiguchi

yhashi@med.teikyo-u.ac.jp

Extended author information available on the last page of the article in diagnostic methods has led to a steady improvement in the results of treatment. However, there are differences in treatment among medical institutions in Japan that provide medical care for patients with colorectal cancer, and the differences may lead to differences in the results of treatment.

Under such circumstances, the JSCCR guidelines 2019 for the treatment of colorectal cancer (JSCCR guidelines 2019), which are intended for doctors (general practitioners and specialists) who provide medical care for patients with colorectal cancer in various disease stages and conditions, have been prepared for the following purposes: (1) to show standard treatment strategies for colorectal cancer, (2) to eliminate disparities among institutions in terms of 
treatment, (3) to eliminate unnecessary treatment and insufficient treatment, and (4) to deepen mutual understanding between healthcare professionals and patients by making these guidelines available to the general public [1].

The following are expected to be achieved with these guidelines: (1) improvement of the treatment of colorectal cancer in Japan; (2) improvement of the results of treatment; (3) reduction of the human and financial burden; and (4) increased benefits for patients.

\section{How to use these guidelines}

These guidelines were prepared by consensuses reached by the Guideline Committee of the Japanese Society for Cancer of the Colon and Rectum, based on a careful review of the evidence retrieved by the literature searches and in view of the medical health insurance system and actual clinical practice settings in Japan and, therefore, these guidelines can be used as a tool for treating colorectal cancer in actual clinical practice settings. More specifically, they can be used as a guide to obtaining informed consent from patients and choosing the method of treatment for each patient. However, these guidelines provide only general recommendations for choosing treatment strategies for colorectal cancer, and they do not control or limit treatment strategies or treatment methods that are not described herein. They can also be used as a document to explain the rationale for selecting treatment strategies and treatment methods that differ from those described therein.

The Japanese Society for Cancer of the Colon and Rectum (JSCCR) is responsible for the statements in these guidelines. However, the personnel directly in charge of treatment, not the JSCCR or the Guideline Committee, are responsible for the outcome of treatment.

\section{Users}

The users of these guidelines are mainly clinical doctors engaged in all aspects of the medical treatment of colorectal cancer.

\section{How to develop these guidelines}

\section{(1) Recording methods}

We adopted the concept from the first edition, in which the treatment policy algorithm was disclosed, a simple explanation thereof recorded, and added further comments with regard to categories requiring additional explanation. Since the 2009 edition, areas of debate have been raised as clinical questions (CQs) and included with recommendations added. In the 2016 edition, systemic therapy was the only treatment to be revised. In the 2019 edition, all aspects of the treatments were revised, with corrections and additions made to the CQs based on knowledge acquired since the 2016 version (systemic therapy) and the 2014 version (other treatments).

Efforts were made to make the expression of the CQs clear and unambiguous. When comparing multiple interventions, we did not stick to ranking everything, and kept the expression flexible to ensure that it is useful in clinical practice. The clinicopathological terms conformed to those described in the "Japanese Classification of Colorectal, Appendiceal, and Anal Carcinoma, third English edition [2].

\section{(2) Evidence level/strength of recommendations of CQs}

The recommendations added to CQs included the evidence level and strength of recommendations determined using the following direction.

\section{(2-1) Evidence level}

Papers relating to the CQs were comprehensively collected, and the evidence indicated by individual papers relating to the critical outcomes included within the CQs was divided into groups by study design [3]. The literature level and a body of evidence (Table 1) were evaluated in reference to the GRADE* System [4-26], before determining the final CQ evidence level (Table 2).

*GRADE: The Grading of Recommendations Assessment, Development and Evaluation

\section{(2-2) Strength of recommendations}

Draft recommendation statements and the strength of the recommendations were directed based on the outcomes and the level of evidence obtained from the process described above and were evaluated at a consensus meeting of the Guideline Committee. In the CQ text, the recommendations that were decided have been directly expressed, and ambiguous expressions were excluded.

The draft recommendations were evaluated from four categories (1) Quality of evidence, (2) Patients' views and preferences, (3) Benefits and harms, and (4) Cost effectiveness). The strength of recommendation (Table 3 ) was determined by vote, based on the GRADE Grid method [11].

\section{Method}

1. We selected one of the following five options and voted.

(1) Strong "For" intervention

(2) A Weak "For" intervention

(3) Weak "Against" intervention

(4) Strong "Against" intervention

(5) Not graded 
Table 1 Rating the quality of evidence

Step 1 (evaluation of individual study): study design, evaluation of bias risk, create structured abstract

Step 2 (overall rating for each important outcome across studies):

1. Initial quality of a body of evidence: evaluation of each study design group

- systematic reviews, meta-analysis, randomized controlled trials = "initial quality A (high level)"

- observation studies, cohort studies, case control studies = "initial quality C (low level)"

" case series, case reports = "initial quality D (very low level)"

2. Five reasons to possibility rate down the quality

- risk of bias

- inconsistency in results

- indirectness of evidence

- data imprecision

- high possibility of publication bias

3. Three reasons to possibility rate up the quality

- large effect with no confounding factors

- dose-response gradient

- possible confounding factors are weaker than actual effects

4. We evaluate 1->2->3, and assess the quality of a body of evidence.

Table 2 Definition of levels of evidence (Ref. [14])

A (high) We are very confident in the effect estimate
$\begin{aligned} & \text { B (moderate) are moderately confident in the effect estimate: the true effect is likely to be close to the estimate of the effect, but there is a } \\ & \text { possibility that it is substantially different }\end{aligned}$
$\begin{aligned} & \text { Our confidence in the effect estimate is limited: the true effect may be substantially different from the estimate of the effect } \\ & \text { D (very low) }\end{aligned}$
$\begin{aligned} & \text { We have very little confidence in the effect estimate: the true effect is likely to be substantially different from the estimate of } \\ & \text { effect }\end{aligned}$

Table 3 Strength of recommendation (Ref. [25])

Strength of recommendation

1 (Strong recommendation)

Strong "For" an intervention

Strong "Against" an intervention

2 (Weak recommendation)

Weak "For" an intervention

Weak "Against" an intervention

2. With one vote, if $70 \%$ or more of the votes were obtained in any of (1) to (5), it was considered a final decision.

If this criterion cannot be met, then the following shall be applied:

- If (1)+(2) exceeds $50 \%$, (3)+(4) is $20 \%$ or lower, "weakly recommend to perform."

- If (3) +(4) exceeds $50 \%$, (1)+ (2) is $20 \%$ or lower, "weakly recommend not to perform."
3. Items not reaching consensus after a single vote were debated once again, with the results of the first vote disclosed and additional information on the situation relating to clinical practice in Japan provided, and discussion and voting was repeated.

4. If agreement was not reached, even in the second vote, no strength of recommendation was presented in the CQ.

\section{Literature search}

At first, the literature search was performed for the clinical questions. Then, a further search was done as needed with additional search techniques.

To survey the latest literature, in addition to the papers used for reference in the previous edition, the PubMed and Ichushi-Web databases were selected for the search, and the English and Japanese literature was searched in both databases from June 2012 to February 2017. However, the start of the search period for systemic therapy was August 2016. The task of searching was performed by a medical librarian, 
who created a search formula based on a discussion with the Committee members in charge of each item and collected literature during the search period. In addition, secondary sources such as UpToDate and literature collected by manual searching were added and critically examined as needed, and other documents such as proceedings and guidelines were included as necessary. We selected 3,295 documents from among the 16,341 documents (PubMed 9,672, ICHUSHI 6,153 , hand search 516) collected during the literature search and critically reviewed all of them (Table 4).

Treatment guidelines for colorectal cancer

Chapter 1: Treatment strategies for Stage 0 to Stage III colorectal cancer

\section{Endoscopic treatment (Fig. 1)}

General principles underlying the indications for endoscopic resection

- There is little possibility of lymph node metastasis, and the size and location of the tumor make en bloc resection possible.

Indication criteria for endoscopic resection:

(1) Intramucosal carcinoma or carcinoma with slight submucosal invasion

(2) Size does not matter

(3) Any macroscopic type

- Endoscopic treatment is a method of endoscopically resecting lesions in the large bowel and of collecting the resected specimens.

- Endoscopic treatment methods consist of polypectomy (note 1), endoscopic mucosal resection (EMR) (note 2), and endoscopic submucosal dissection (ESD) (note 3).

- In determining the indication for endoscopic treatment and the treatment method, information on the size, predicted depth of invasion, and morphology of the tumor is essential.

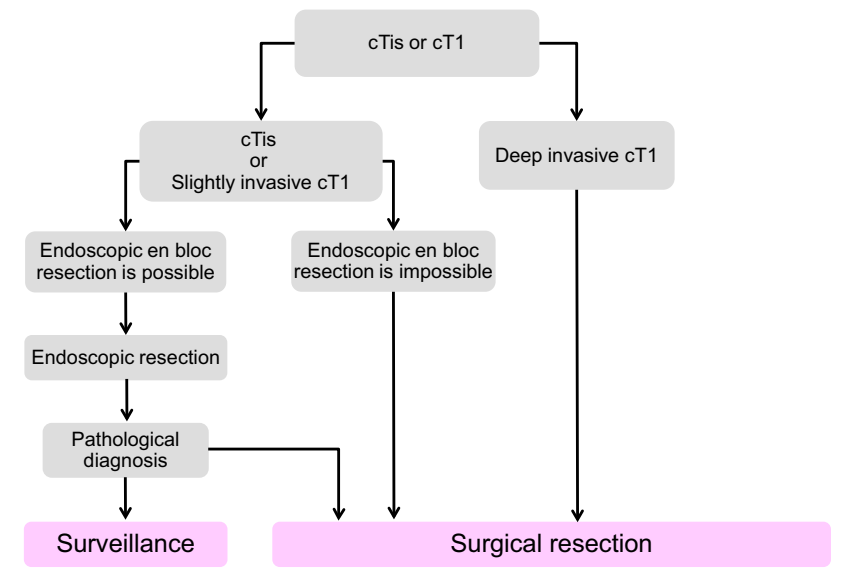

Fig. 1 Treatment strategies for cTis and cT1 colorectal cancer

\section{Comments}

(1) Endoscopic resection is intended for both diagnosis and treatment. It consists of total excisional biopsy in which curability and the necessity of additional intestinal resection are assessed by histopathological examination of the resected specimens (CQ-1).

(2) cT1 deeply invasive cancer is diagnosed based on endoscopic findings, such as "fullness, erosion, ulcer, fold convergence, deformity, rigidity," as well as contrast $\mathrm{X}$-ray, chromoendoscopy, image-enhanced endoscopy (e.g., NBI/BLI [27], or magnifying endoscopic observation) and endoscopic ultrasound findings. [28-30].

(3) En bloc resection is desirable for accurate diagnosis of the status of carcinoma invasion in the resection margin and the deepest area.

- $2 \mathrm{~cm}$ is the largest size of a tumor that can be easily resected en bloc by polypectomy or snare EMR [31] (CQ-2).

- Colorectal ESD is an "endoscopic resection technique which enables en bloc resection of a tumor, regardless of size," which was approved for implementation
Table 4 Number of scientific articles retrieved and selected

\begin{tabular}{|c|c|c|c|c|c|}
\hline & \multicolumn{2}{|c|}{$\begin{array}{l}\text { Number of articles } \\
\text { retrieved }\end{array}$} & \multicolumn{2}{|c|}{$\begin{array}{l}\text { Number of articles } \\
\text { selected }\end{array}$} & \multirow[t]{2}{*}{$\begin{array}{l}\text { Number of articles } \\
\text { retrieved manually }\end{array}$} \\
\hline & PubMed & Ichushi & PubMed & Ichushi & \\
\hline (1) Endoscopic treatment & 1102 & 539 & 136 & 73 & 81 \\
\hline (2) Surgical treatment & 3351 & 2521 & 926 & 192 & 82 \\
\hline (3) Radiotherapy & 1225 & 181 & 271 & 16 & 67 \\
\hline (4) Systematic therapy & 2019 & 1381 & 591 & 108 & 242 \\
\hline (5) Others & 1975 & 1530 & 374 & 86 & 44 \\
\hline Total & 9672 & 6153 & 2304 & 475 & 516 \\
\hline
\end{tabular}


under health insurance in April 2012 with regard to "early-stage malignant tumors". Given the high likelihood of technically difficult complications (perforations), however, it should only be implemented after sufficient consideration of the level of skill of the endoscopist performing the procedure. Tumors with a diameter between 2 and $5 \mathrm{~cm}$ were covered by insurance. The revision of April 2018 eliminated the upper limit of the tumor diameter and the indication became early colon cancer with a maximum diameter of $2 \mathrm{~cm}$ or more. Early colon cancer accompanying fibrosis is even applied to tumors with a diameter of $2 \mathrm{~cm}$ or less (CQ-2).

- EMRC (EMR using a cap) is reported to involve a high risk of perforation when used for colon lesions.

- If the preoperative diagnosis is cancer accompanied by adenoma (intramucosal carcinoma), a piecemeal resection can be performed with regard to the adenoma, while avoiding division of the cancerous area. It should be noted, however, that piecemeal resection is associated with a high incomplete resection rate and a high local recurrence rate. Multiple-piecemeal resection, which makes accurate histological judgment difficult, should be avoided [31].

- After endoscopic resection, the resection margin should be observed in detail and the presence or absence of a residual lesion should be confirmed.

- Dye spray and magnifying observation are useful for the diagnosis of residual lesions [30].

- If residual mucosal lesions are present, additional treatment (e.g., endoscopic additional resection, hot biopsy, cautery, etc.) should be performed.

(4) Follow-up observation after endoscopic treatment

- For piecemeal resection of pTis carcinoma with a positive horizontal margin, the presence or absence of local recurrence is investigated by colonoscopy at around 6 months (CQ-3).

- For follow-up observation of pT1 cancer, a search not only for local recurrence but also lymph node recurrence and distant metastasis recurrence is necessary, with follow-ups including endoscopic examinations, image diagnoses such as CT examinations and tumor markers (CQ-3).

- While recurrence after endoscopic treatment for pT1 cancer is often within three years, caution is required as it may also recur thereafter [32].

Note 1 Polypectomy-In this technique, a snare is placed on the stalk of the lesion, and the lesion is electrocauterized using a high-frequency current. This method is mainly used for protruding lesions.

Note 2 EMR - In this technique, the lesion is elevated by local injection of a liquid such as physiological saline into the submucosa, and the lesion is electrocauterized the same as in case of polypectomy. This method includes the snare method and EMR using a cap (EMRC). It is mainly used for superficial tumors and large sessile lesions.

Note 3 ESD-In this technique, the lesion is elevated by local injection of a liquid such as sodium hyaluronate solution into the submucosa of the perilesional area; then, circumferential incision of the mucosa surrounding the lesion and dissection of the submucosa with a special knife, and en bloc resection are performed [33]. ESD is mainly indicated for large tumors, especially for early cancers, that cannot be resected by en bloc EMR

Note 4 Precutting EMR - In this technique, snaring is performed without dissecting the submucosal layer after incising the circumference of the lesion alone, using a knife for ESD or the tip of a snare.

Note 5 Hybrid ESD-In this technique, the submucosal layer is dissected and snaring is carried out after the ESD procedure (mucosal incision + submucosal dissection, using a knife for ESD or the tip of a snare).

\section{Surgical treatment (Fig. 2)}

Principles of surgery

- The extent of lymph node dissection to be performed during colorectal cancer surgery is determined based on the preoperative clinical findings and on the extent of lymph node metastasis and depth of tumor invasion by the tumor observed intraoperatively.

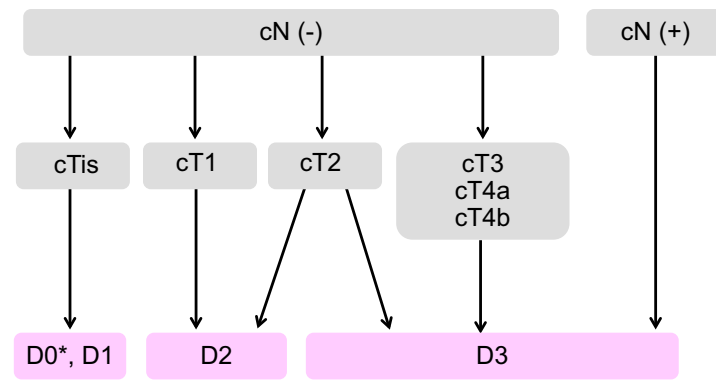

*Includes local rectal resection for rectal cancer

Fig. 2 Surgical treatment strategies for cStage 0 to cStage III colorectal cancer 
- If lymph node metastasis is recognized, or suspected based on the preoperative/intraoperative findings, D3 dissection is performed [34].

- If no lymph node metastases are observed based on the preoperative/intraoperative diagnostic findings, lymph node dissection is performed based on the depth of tumor invasion [35].

(1) Lymph node dissection is unnecessary for pTis cancer (D0), because pTis cancer is not accompanied by lymph node metastasis. However, D1 dissection can be performed when bowel resection is adopted.
(2) D2 dissection is necessary for pT1 cancer, because the incidence of lymph node metastasis is approximately $10 \%$ and because approximately $2 \%$ of pT 1 cancer is accompanied by intermediate lymph node metastasis (Table 5).

(3) Although there is insufficient evidence describing the extent of lymph node dissection for cT2 (MP) cancer, at least D2 dissection is necessary. However, D3 dissection can be performed, because about $1 \%$ of cT2 (MP) cancer is accompanied by main lymph node metastases (Table 5) and because preoperative diagnosis of depth of invasion is not very accurate.
Table 5 Incidences of lymph node metastasis according to tumor location and depth of tumor invasion

\begin{tabular}{|c|c|c|c|c|c|c|c|}
\hline & & \multirow[t]{2}{*}{ No. of patients } & \multicolumn{5}{|c|}{ Extent of lymph node metastasis detected histologically } \\
\hline & & & $n_{0}(\%)$ & $n_{1}(\%)$ & $n_{2}(\%)$ & $n_{3}(\%)$ & $n_{4}(\%)$ \\
\hline \multirow[t]{6}{*}{ All sites } & $\mathrm{sm}$ & 3151 & 90.7 & 7.3 & 1.9 & 0.0 & 0.1 \\
\hline & $\mathrm{mp}$ & 3590 & 77.3 & 17.4 & 4.2 & 0.9 & 0.3 \\
\hline & ss $/ a_{1}$ & 11,272 & 54.6 & 29.9 & 12.0 & 2.3 & 1.2 \\
\hline & $\mathrm{se} / \mathrm{a}_{2}$ & 6101 & 35.9 & 34.4 & 20.2 & 5.7 & 3.8 \\
\hline & si/ai & 1502 & 43.0 & 27.6 & 16.4 & 6.7 & 6.3 \\
\hline & Total & 25,617 & 57.1 & 26.3 & 11.9 & 2.9 & 1.9 \\
\hline \multirow[t]{6}{*}{ Colon } & $\mathrm{sm}$ & 1957 & 91.4 & 6.8 & 1.8 & 0.0 & 0.0 \\
\hline & $\mathrm{mp}$ & 1747 & 79.3 & 16.3 & 3.5 & 0.6 & 0.3 \\
\hline & $\mathrm{ss} / \mathrm{a}_{1}$ & 7333 & 56.6 & 28.1 & 11.7 & 2.4 & 1.2 \\
\hline & $\mathrm{se} / \mathrm{a}_{2}$ & 3363 & 37.4 & 34.0 & 19.3 & 5.6 & 3.7 \\
\hline & si/ai & 960 & 44.6 & 28.6 & 14.7 & 5.5 & 6.6 \\
\hline & Total & 15,360 & 58.6 & 25.4 & 11.3 & 2.8 & 1.8 \\
\hline \multirow[t]{6}{*}{ Rectosigmoid } & $\mathrm{sm}$ & 337 & 88.7 & 9.5 & 1.8 & 0.0 & 0.0 \\
\hline & $\mathrm{mp}$ & 429 & 80.4 & 17.0 & 2.6 & 0.0 & 0.0 \\
\hline & $\mathrm{ss} / \mathrm{a}_{1}$ & 1584 & 53.9 & 33.0 & 10.2 & 1.3 & 1.7 \\
\hline & $\mathrm{se} / \mathrm{a}_{2}$ & 789 & 34.2 & 38.4 & 20.8 & 3.2 & 3.4 \\
\hline & si/ai & 187 & 44.9 & 24.6 & 19.3 & 4.8 & 6.4 \\
\hline & Total & 3326 & 55.7 & 29.3 & 11.4 & 1.6 & 2.0 \\
\hline Upper and & $\mathrm{sm}$ & 839 & 89.7 & 7.7 & 2.0 & 0.1 & 0.4 \\
\hline \multirow[t]{5}{*}{ lower rectum } & $\mathrm{mp}$ & 1373 & 73.9 & 19.2 & 5.4 & 1.4 & 0.1 \\
\hline & ss $/ \mathrm{a}_{1}$ & 2310 & 48.8 & 33.3 & 14.2 & 2.7 & 1.0 \\
\hline & $\mathrm{se} / \mathrm{a}_{2}$ & 1904 & 33.9 & 33.6 & 21.5 & 6.8 & 4.1 \\
\hline & si/ai & 328 & 38.1 & 26.2 & 19.8 & 10.4 & 5.5 \\
\hline & Total & 6754 & 54.3 & 27.0 & 13.3 & 3.6 & 1.8 \\
\hline \multirow[t]{6}{*}{ Anal canal } & $\mathrm{sm}$ & 18 & 94.4 & 0.0 & 5.6 & 0.0 & 0.0 \\
\hline & $\mathrm{mp}$ & 41 & 70.7 & 9.8 & 7.3 & 7.3 & 4.9 \\
\hline & $\mathrm{ss} / \mathrm{a}_{1}$ & 45 & 60.0 & 22.2 & 8.9 & 6.7 & 2.2 \\
\hline & $\mathrm{se} / \mathrm{a}_{2}$ & 46 & 32.6 & 21.7 & 23.9 & 15.2 & 6.5 \\
\hline & si/ai & 27 & 33.3 & 25.9 & 14.8 & 18.5 & 7.4 \\
\hline & Total & 177 & 54.8 & 17.5 & 13.0 & 10.2 & 4.5 \\
\hline
\end{tabular}

(JSCCR colorectal cancer registry: patients in years 2000-2004) Depth of invasion and the degree of lymph node metastasis were determined according to the rules set forth in the "Japanese Classification of Colorectal Carcinoma" (6th edition). sm submucosa, $m p$ muscularis propria, ss subserosa, se serosa, al shallow part of adventitia, $a 2$ deeper part of adventitia, si/ai direct invasion of other organs through the serosa/adventitia 
For details of lateral lymph node dissection in rectal cancer, see (CQ-5).

Surgical treatment for rectal cancer:

- The principle for radical surgery for rectal cancer is TME (total mesorectal excision) or TSME (tumor-specific mesorectal excision) [36-39].

[Indication criteria for sphincter preserving surgery]

- Sphincter preserving surgery is indicated only when the following criteria are fulfilled: (i) resection with no oncologic remnant (both the distal and circumferential resection margins are negative $=\mathrm{DM} 0, \mathrm{RM} 0$ ) can be achieved, and (ii) the postoperative anal function can be maintained.

[Autonomic nerve-preserving surgery]

- Considering factors such as the degree of cancer progression and the presence or absence of macroscopic nerve invasion, preservation of autonomic nerves is attempted to preserve urinary and sexual functions as much as possible, provided that curability is unaffected.
[Indications criteria for lateral lymph node dissection]

- Lateral lymph node dissection is indicated when the lower border of the tumor is located distal to the peritoneal reflection and the tumor has invaded beyond the muscularis propria [40] (Table 6) (CQ-5).

Laparoscopic surgery:

- The indications for laparoscopic surgery are determined by considering the surgeon's experience and skills as well as tumor factors, such as the location and degree of progression of the cancer, and patient factors, such as obesity and history of open abdominal surgery (CQ-4).

\section{Comments}

[Optimal length of the bowel resection]

(1) In D1, D2, D3 dissection, the resection margin of the bowel is determined so that the pericolic/perirectal lymph node, as defined in Japanese Classification of Colorectal, Appendiceal, and Anal Carcinoma [2], is dissected.

(2) The extent of the pericolic/perirectal lymph node in colon cancer is defined by the positional relationship

Table 6 Lateral dissection and lateral metastasis of rectal cancer

\begin{tabular}{|c|c|c|c|c|c|c|c|}
\hline & & No. of patients & $\begin{array}{l}\text { No. of patients who } \\
\text { underwent lateral dis- } \\
\text { section }\end{array}$ & $\begin{array}{l}\text { Lateral dis- } \\
\text { section rate } \\
(\%)\end{array}$ & $\begin{array}{l}\text { No. of patients } \\
\text { with lateral metas- } \\
\text { tasis }\end{array}$ & $\begin{array}{l}\text { Lateral metastasis } \\
\text { rate (percentage of all } \\
\text { patients) }(\%)\end{array}$ & $\begin{array}{l}\text { Lateral metastasis rate } \\
\text { (percentage of patients } \\
\text { who underwent lateral } \\
\text { dissection) }(\%)\end{array}$ \\
\hline \multirow[t]{6}{*}{ RS } & $\mathrm{sm}$ & 124 & 0 & 0 & 0 & 0.0 & 0.0 \\
\hline & $\mathrm{mp}$ & 127 & 6 & 4.7 & 0 & 0.0 & 0.0 \\
\hline & $\mathrm{ss} / \mathrm{a}_{1}$ & 316 & 24 & 7.5 & 0 & 0.0 & 0.0 \\
\hline & $\mathrm{se} / \mathrm{a}_{2}$ & 177 & 8 & 4.5 & 0 & 0.0 & 0.0 \\
\hline & si/ai & 32 & 14 & 43.8 & 1 & 3.1 & 7.1 \\
\hline & Total & 776 & 52 & 6.7 & 1 & 0.1 & 1.9 \\
\hline \multirow[t]{6}{*}{$\mathrm{Ra}$} & $\mathrm{sm}$ & 138 & 5 & 3.6 & 0 & 0.0 & 0.0 \\
\hline & $\mathrm{mp}$ & 149 & 18 & 12.1 & 0 & 0.0 & 0.0 \\
\hline & $\mathrm{ss} / \mathrm{a}_{1}$ & 230 & 58 & 25.2 & 4 & 1.7 & 6.9 \\
\hline & $\mathrm{se} / \mathrm{a}_{2}$ & 181 & 59 & 32.6 & 7 & 3.9 & 11.9 \\
\hline & si/ai & 15 & 8 & 53.3 & 0 & 0.0 & 0.0 \\
\hline & Total & 713 & 148 & 20.8 & 11 & 1.5 & 7.4 \\
\hline \multirow[t]{6}{*}{$\mathrm{RaRb}+\mathrm{Rb}$} & $\mathrm{sm}$ & 234 & 37 & 15.8 & 2 & 0.9 & 5.4 \\
\hline & $\mathrm{mp}$ & 372 & 218 & 58.6 & 20 & 5.4 & 9.2 \\
\hline & $\mathrm{ss} / \mathrm{a}_{1}$ & 350 & 230 & 65.7 & 28 & 7.7 & 12.2 \\
\hline & $\mathrm{se} / \mathrm{a}_{2}$ & 412 & 319 & 77.4 & 75 & 18.0 & 23.5 \\
\hline & si/ai & 59 & 48 & 81.4 & 17 & 28.8 & 35.4 \\
\hline & Total & 1427 & 852 & 59.7 & 142 & 9.8 & 16.7 \\
\hline
\end{tabular}

(Project study by the JSCCR: patients in years 1991-1998). $R S$ rectosigmoid, $R a$ upper rectum, $R b$ lower rectum 
between the primary tumor and the feeding artery. Metastasis of the pericolic/perirectal lymph node at a distance of $10 \mathrm{~cm}$ or more from the tumor edge is rare [41]. Currently, as a JSCCR research project, a multicenter cohort study investigating the distance between metastasis-positive pericolic/perirectal lymph node and the primary tumor is ongoing.

(3) The extent of the pericolic/perirectal lymph nodes in rectal cancer is defined as follows: the oral side is defined by the lowest plunge point of the sigmoid artery, while the anal side is defined by the distance from the tumor edge. For cStage 0-III cases, it is rare for intramural and/or mesorectal distal cancer spread to develop at a distance of $3 \mathrm{~cm}$ or more from the tumor edge in RS and $\mathrm{Ra}$ cancer, or $2 \mathrm{~cm}$ or more in $\mathrm{Rb}$ cancer [42-45]. Thus, the distal resection margin of the bowel and mesorectum should be determined to include this range.

(4) It should be noted that pT4, pN2, M1 (Stage IV), and poorly differentiated rectal cancer cases are frequently accompanied by distal spread a long distance from the primary tumor edge [41, 43-45].

\section{[TME/TSME]}

- Total mesorectal excision (TME) is a procedure that resects all the mesorectum just above the anal canal [36]. Tumor-specific mesorectal excision (TSME) is a procedure for partially resecting the mesorectum according to the location of the tumor [39].

\section{[Intersphincteric resection (ISR)]}

- ISR is a procedure for lower rectal cancer located close to the anus, to ensure the adequate distal margin via the removal of the internal anal sphincter and to avoid a permanent stoma.

- The indication criteria for ISR are as follows: (1) able to ensure the resection with clear circumferential surgical resection margin (no infiltration to the external anal sphincter or levator ani muscles); and (2) able to ensure the adequate distal surgical margin (in general, $2 \mathrm{~cm}$ or more for T2/T3 tumors and $1 \mathrm{~cm}$ or more for $\mathrm{T} 1$ tumors). ISR is not recommended for cases with poorly differentiated cancer and cases in which the anal sphincter tonus is decreased.

- In a systematic review of 14 papers, the $\mathrm{R} 0$ resection rate of patients who underwent ISR was $97.0 \%$, the anastomotic leakage rate was $9.1 \%$, and the local recurrence rate was $6.7 \%$, which is reported as an acceptable result [46]. However, according to the questionnaire survey conducted by the JSCCR in 2125 cases, the 5-year survival rate of patients who underwent ISR was equivalent to that of the lower rectal cancer cases in the JSCCR colorectal cancer registry, but the 5-year local recurrence rate (including recurrence in the area of anastomosis) was relatively high at $11.5 \%$. Obviously, the local recurrence rate becomes higher as the depth of invasion reaches deeper $(4.2 \%$ at $\mathrm{T} 1,8.5 \%$ at $\mathrm{T} 2,18.1 \%$ at $\mathrm{T} 3$, and $36.0 \%$ at T4). The indication of ISR should be determined based on a precise preoperative diagnosis of the tumor depth.

- As the extent of resection of the anal sphincter becomes wider, postoperative defecatory dysfunction (e.g., fecal incontinence) becomes a more serious problem. In particular, it has been reported that the incidence of defecatory dysfunction is high in patients who receive preoperative radiation therapy, those with anastomotic leakage, and the elderly [47-49].

- The indication of ISR should be carefully decided because the procedure is associated with a high degree of difficulty and has a great influence on the patient's QOL, including the postoperative defecatory function. In addition to tumor factors (e.g., the histological type and depth), and patient factors (e.g., age and sphincter tonus), the experience and skill of the operator should be taken into consideration.

\section{[Autonomic nerve-preserving surgery]}

- The autonomic nervous system related to surgery for rectal cancer consists of the lumbar splanchnic nerves*, superior hypogastric plexus*, hypogastric nerves*, pelvic splanchnic nerves\#, and pelvic plexus. (*sympathetic nerves, \#parasympathetic nerves)

- Regarding the urinary function, if one side of the pelvic nerve plexus is preserved [AN 1-4], a certain function is maintained.

- The hypogastric nerve controls the ejaculation function, and the internal pelvic nerve governs the erectile function. To maintain the male sexual function, full conservation of the autonomic nervous system on both sides [AN 4] is necessary.

- The urinary function and male sexual function may be impaired even if the autonomic nervous system is fully preserved, regardless of whether lateral lymph node dissection is performed or not [50-52].

\section{[Local excision for rectal cancer]}

- Local excision is indicated for cTis cancer and cT1 cancer (slight invasion) located distal to the second Houston valve (peritoneal reflection).

- Histological investigation of the resected specimen allows a determination to be made of the likelihood that 
treatment will cure the condition completely, along with the need for additional treatment (intestinal resection accompanied by lymph node dissection).

[Aggregate data from the JSCCR colorectal cancer registry]

(1) The incidence of lymph node metastasis according to site and depth of tumor invasion, curative resection rate, and 5-year survival rate are shown in Tables 5, 7, and 8 [35].

(2) The 5-year survival rates after curative resection of pStage 0 to pStage III colorectal cancer according to site were: All sites: $82.2 \%$, Colon: $83.8 \%$, Rectosigmoid: $81.7 \%$, Ra- $\mathrm{Rb}$ rectum: $79.3 \%$ (patients in years 2000 2004).

Chapter 2: Treatment strategies for Stage IV colorectal cancer (Fig. 3)

- Stage IV colorectal cancer is associated with synchronous distant metastasis to any of the following organs: liver, lung, peritoneum, brain, distant lymph nodes, or other organ (e.g., bone, adrenal gland, spleen).

- If both the distant metastases and the primary tumor are resectable, curative resection of the primary tumor is performed, and resection of the distant metastases is considered.

- If the distant metastases are resectable but the primary tumor is unresectable, in principle, resection of the primary tumor and distant metastases is not performed, and another treatment method is selected.

- If the distant metastases are unresectable but the primary tumor is resectable, the indication for the resection of the primary tumor is determined, based on the clinical symptoms of the primary tumor and the impact on the prognosis (CQ-6).

\section{Comments}

(1) The incidence of synchronous distant metastasis is shown in Table 9.

(2) Liver metastases

- If resectable, liver metastases should be resected upon confirming the radicality of the primary resection.

- As for the timing of resection, simultaneous resection of the primary lesion and liver metastases can be safely performed [53]. Depending on the difficulty of hepatectomy and the general condition of the patient, metachronous resection is also performed. However, it is unclear whether simultaneous resection or metachronous resection improves the longterm prognosis.

(3) Lung metastases

- If resectable, resection of lung metastases should be considered after resection of the primary tumor.

- Metachronous resection is generally performed to remove lung metastases after primary resection.

(4) Peritoneal metastases (CQ-7)

- Complete resection is strongly recommended for P1.

- Complete resection is recommended for P2 when easily resectable.
Table 7 Curative resection rate according to $\mathrm{pStage}$ (lower rows: no. of patients)

\begin{tabular}{lllllll}
\hline pStage & I & II & IIIa & IIIb & IV & All stages \\
\hline All patients & $98.7 \%$ & $96.2 \%$ & $91.9 \%$ & $81.8 \%$ & - & $78.0 \%$ \\
& 5455 & 7336 & 5635 & 2572 & 4300 & 25,298 \\
Colon & $99.1 \%$ & $96.6 \%$ & $92.4 \%$ & $83.6 \%$ & - & $77.2 \%$ \\
& 3028 & 4688 & 3208 & 1379 & 2787 & 15,090 \\
Rectosigmoid & $99.5 \%$ & $96.6 \%$ & $92.5 \%$ & $80.2 \%$ & - & $78.0 \%$ \\
\multirow{3}{*}{ Upper and lower rectum } & 615 & 961 & 835 & 288 & 560 & 3259 \\
& $97.9 \%$ & $95.0 \%$ & $90.9 \%$ & $80.5 \%$ & - & $79.9 \%$ \\
Anal canal & 1764 & 1644 & 1564 & 866 & 929 & 6767 \\
& $95.8 \%$ & $86.0 \%$ & $78.6 \%$ & $61.5 \%$ & - & $70.9 \%$ \\
\hline
\end{tabular}

(JSCCR colorectal cancer registry: patients in years 2000-2004)

Curative resection rate $=$ Number of patients with histological curability A cancer/Total number of patients who underwent surgery

Staging was performed according to the rules set forth in the "Japanese Classification of Colorectal Carcinoma" (6th edition) 
Table 8 Cumulative 5-year survival rate according to tumor location (lower rows: no. of patients)

Fig. 3 Treatment strategies for Stage IV colorectal cancer

\begin{tabular}{llllllll}
\hline pStage & 0 & I & II & IIIa & IIIb & IV & All Stages \\
\hline Cecum & $91.0 \%$ & $93.7 \%$ & $83.5 \%$ & $73.0 \%$ & $65.4 \%$ & $12.5 \%$ & $68.2 \%$ \\
& 79 & 185 & 249 & 207 & 113 & 204 & 1037 \\
Ascending colon & $93.9 \%$ & $91.2 \%$ & $85.8 \%$ & $79.1 \%$ & $63.4 \%$ & $19.1 \%$ & $71.4 \%$ \\
& 125 & 338 & 656 & 416 & 211 & 410 & 2156 \\
Transverse colon & $88.9 \%$ & $91.4 \%$ & $85.2 \%$ & $78.5 \%$ & $65.7 \%$ & $20.8 \%$ & $74.0 \%$ \\
& 105 & 277 & 428 & 244 & 138 & 210 & 1402 \\
Descending colon & $100.0 \%$ & $94.1 \%$ & $85.3 \%$ & $82.0 \%$ & $52.9 \%$ & $21.1 \%$ & $75.4 \%$ \\
& 43 & 146 & 224 & 166 & 52 & 117 & 748 \\
Sigmoid colon & $94.2 \%$ & $92.3 \%$ & $85.8 \%$ & $83.0 \%$ & $64.7 \%$ & $22.0 \%$ & $73.7 \%$ \\
& 154 & 852 & 1124 & 837 & 363 & 736 & 4066 \\
Rectosigmoid & $89.4 \%$ & $91.5 \%$ & $84.8 \%$ & $78.0 \%$ & $60.0 \%$ & $19.8 \%$ & $71.6 \%$ \\
Upper rectum & 54 & 366 & 539 & 473 & 175 & 322 & 1929 \\
& $98.0 \%$ & $95.3 \%$ & $84.6 \%$ & $75.9 \%$ & $57.7 \%$ & $11.6 \%$ & $72.4 \%$ \\
Lower rectum & 67 & 356 & 464 & 471 & 173 & 263 & 1794 \\
& $97.5 \%$ & $88.3 \%$ & $81.7 \%$ & $70.0 \%$ & $51.4 \%$ & $11.6 \%$ & $70.5 \%$ \\
Anal canal & 142 & 718 & 486 & 473 & 332 & 298 & 2449 \\
& $100.0 \%$ & $78.7 \%$ & $90.9 \%$ & $46.9 \%$ & $61.2 \%$ & $15.7 \%$ & $60.0 \%$ \\
Colon & 4 & 16 & 14 & 16 & 19 & 17 & 86 \\
Rectum (Ra+ Rb) & $97.6 \%$ & $90.6 \%$ & $83.1 \%$ & $73.0 \%$ & $53.5 \%$ & $14.8 \%$ & $71.3 \%$ \\
& 209 & 1074 & 950 & 944 & 505 & 561 & 4243 \\
& $93.0 \%$ & $92.3 \%$ & $85.4 \%$ & $80.4 \%$ & $63.8 \%$ & $19.9 \%$ & $72.8 \%$ \\
& 506 & 1798 & 2681 & 1870 & 877 & 1677 & 9409 \\
& 773 & 3254 & 4184 & 3303 & 1576 & 2577 & 15,667 \\
\hline
\end{tabular}

(JSCCR colorectal cancer registry: patients in years 2000-2004)

Only adenocarcinomas (including mucinous carcinomas and signet-ring cell carcinomas) were counted Survival rates were calculated by the life table method with death from any cause as an event 5 -year censoring rate $=20.5 \%(3208 / 15,667)$

Staging was performed according to the rules set forth in the "Japanese Classification of Colorectal Carcinoma" (6th edition)

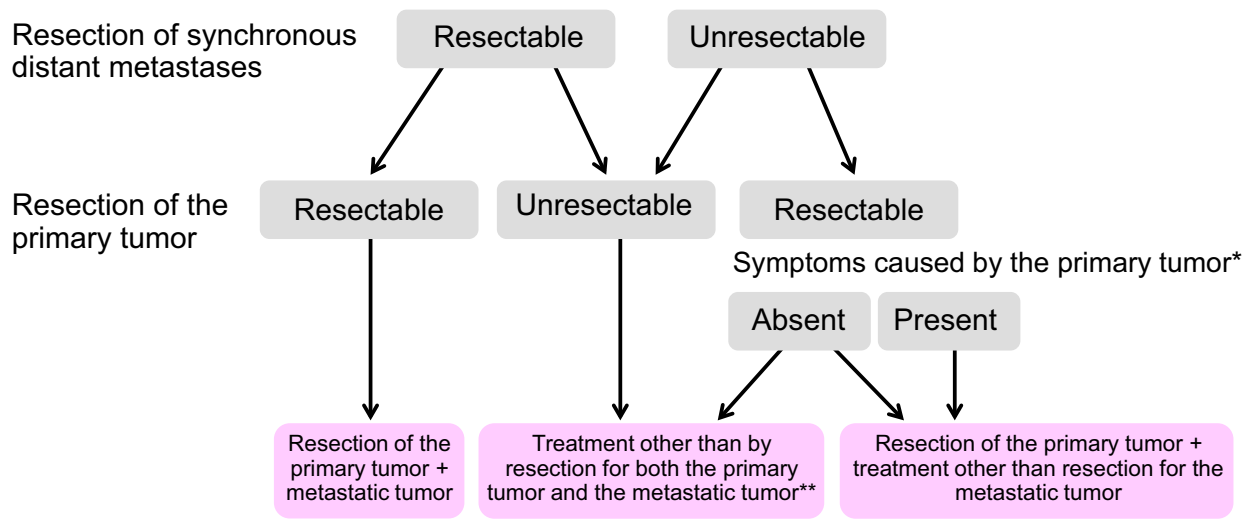

\footnotetext{
* Symptoms caused by the primary tumor: Symptoms caused by events such as massive bleeding, severe anemia, penetration / perforation, and stenosis.

** Treatment other than by resection: Palliative surgery for the primary tumor, chemotherapy, radiotherapy; see "treatment strategies for hematogenous metastasis".
} 
Table 9 Incidence of synchronous distant metastasis of colorectal cancer

\begin{tabular}{llllllllll}
\hline & Liver & Lung & Peritoneum & \multicolumn{2}{l}{ Other sites } \\
\cline { 6 - 10 } & & & & & Bone & Brain & Virchow & Other & Total \\
\hline Colon cancer & $11.8 \%$ & $2.2 \%$ & $5.7 \%$ & $0.3 \%$ & $0.0 \%$ & $0.1 \%$ & $1.3 \%$ & $1.8 \%$ \\
No. of patients 15,391 & 1815 & 338 & 875 & 47 & 6 & 23 & 205 & 281 \\
Rectal cancer & $9.5 \%$ & $2.7 \%$ & $2.6 \%$ & $0.5 \%$ & $0.0 \%$ & $0.1 \%$ & $1.1 \%$ & $1.7 \%$ \\
No. of patients 10,221 & 970 & 273 & 266 & 49 & 5 & 6 & 112 & 172 \\
Total no. of pateints & $10.9 \%$ & $2.4 \%$ & $4.5 \%$ & $0.4 \%$ & $0.0 \%$ & $0.1 \%$ & $1.2 \%$ & $1.8 \%$ \\
25,621 & 2785 & 611 & 1141 & 96 & 11 & 29 & 317 & 453 \\
\hline
\end{tabular}

(JSCCR colorectal cancer registry: patients in years 2000-2004)
- The efficacy of resection of P3 has not been demonstrated.

(5) Distant lymph node metastases

Excision of distant lymph node metastases may be considered, but no comparative clinical trials have shown a clear therapeutic effect. However, in recent years, resection of para-aortic lymph node metastases was reported to have the potential to achieve radical cure and longer survival at certain rates.

Excision of distant lymph node metastases may be considered, but no comparative clinical trials have shown a clear therapeutic effect. However, in recent years, resection of para-aortic lymph node metastases was reported to have the potential to achieve a radical cure and longer survival at certain rates [54-58].

(6) Other distant metastases (bone, brain, adrenal gland, spleen, etc.)

- Although there are reports of resection of these metastatic lesions, no clear effect on survival has been shown.

(7) Cases accompanied by distant metastasis to multiple organs

- Typically, these cases involve metastasis to the liver or lungs.

- If it is safe and simple to remove the primary lesion and the metastasized lesions in the liver or lungs, resection should also be considered [59, 60] (CQ-8).

(8) Adjuvant therapy subsequent to the resection of distant metastasis

- Although evidence is lacking with regard to the efficacy of adjuvant chemotherapy, in view of the high recurrence rate, it is recommended that adjuvant chemotherapy should be performed after the curative resection of distant metastasis (CQ-19).

Chapter 3: Treatment strategies for recurrent colorectal cancer (Fig. 4)

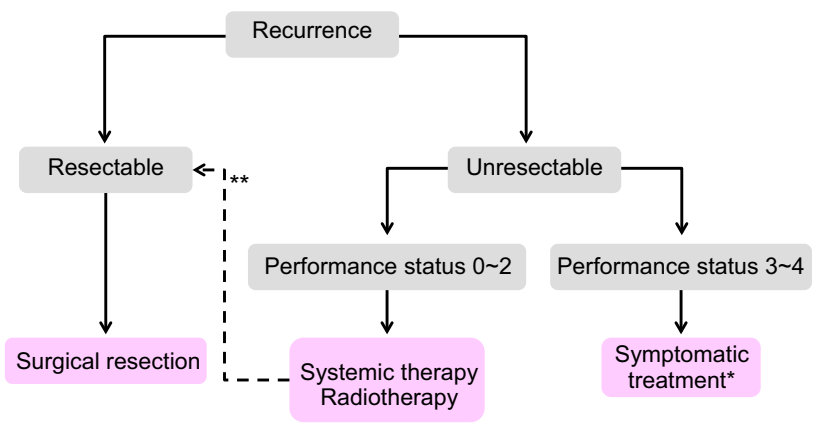

In principle, surgical treatment is indicated for recurrence limited to 1 organ, but it is considered for recurrence in 2 or more organs, if the lesions are resectable. ${ }^{*}$ Best supportive care (BSC).

${ }^{\star *}$ Recurrence may become resectable after successful systemic therapy.

Fig. 4 Treatment strategies for recurrent colorectal cancer

- The goal of treatment for recurrent colorectal cancer is improvement of the prognosis and patient's QOL.

- Treatment methods include surgery, systemic therapy, and radiotherapy. Arterial infusion chemotherapy and thermal ablation therapy are not recommended (CQ-13, 24).

- An appropriate treatment method should be selected with the informed consent of the patient in view of a variety of factors, such as the prognosis, complications, and QOL expected after treatment.

- If recurrence is observed in a single organ and complete surgical resection of the recurrent tumor (s) is possible, resection is strongly considered.

- If recurrence is observed in more than a single organ, resection can be considered if the recurrent tumors in all the organs are resectable $[59,61]$. The efficacy of curative resection in patients who have liver and lung metastases has been shown and, thus, resection should be considered (CQ-8).

- Some authors believe that resection of liver or lung metastases should be performed only after a certain observation period to rule out occult metastases [62, 63].

- Systemic therapy is effective with regard to cases of unresectable liver metastasis, with some cases demonstrat- 
ing that curative resection may become possible $[64,65]$ (CQ-10).

- The efficacy and safety of preoperative chemotherapy for resectable recurrent lesions are not clear, and application should be considered with caution (CQ-9).

- For adjuvant chemotherapy following resection of recurrent colorectal cancer, there is no clear evidence of efficacy with the exception of reports showing that 5-FU or UFT/LV prolongs the relapse-free survival after resection of liver metastasis (CQ-19).

\section{Comments}

[Treatment methods for hematogenous metastases] (See Chapter 4 "Treatment strategies for hematogenous metastases")

[Lymph node recurrence/peritoneal recurrence]

(1) In general, it is reasonable to regard lymph node recurrence or peritoneal recurrence after curative resection of the primary tumor as a part of systemic disease. Thus, systemic therapy should be conducted referring to the section on systemic therapy for unresectable colorectal cancer (See Chapter 5.2. Systemic therapy for unresectable colorectal cancer).

(2) Resection for localized lymph node recurrence or peritoneal recurrence could be considered only when the disease is controlled. However, its efficacy is not clear. The surgical indication should be decided after careful consideration of the risk of surgery and the postoperative quality of life [54, 57, 66-68].

(3) Radiotherapy may be effective for treating localized lymph node recurrence [69-71].

[Local recurrence of rectal cancer]

(1) The extent of spread of the recurrent tumor is evaluated by diagnostic imaging, and resection is considered only for patients in whom complete resection can be expected, after taking into consideration such factors as the pattern of recurrence, symptoms, and physical findings (CQ-14).

(2) The indication for the palliative resection of local recurrence for the purpose of improving survival and providing relief from symptoms should be carefully considered because its effectiveness is not established [72].

(3) If complete resection cannot be expected, systemic therapy is the first choice of treatment from the viewpoint of continuous disease control. However, local effects, such as the alleviation of symptoms, can be expected from radiation therapy. Chemoradiotherapy or radiotherapy can also be a treatment option if symptoms, effects, and adverse events are fully considered [73] (CQ-26).

Chapter 4: Treatment strategies for hematogenous metastases (Fig. 5)

\section{Treatment strategies for liver metastases}

- Treatment of liver metastases is broadly divided into hepatectomy, systemic therapy, hepatic arterial infusion therapy, and thermal ablation therapy.

- Hepatectomy is recommended for liver metastases when curative resection is possible.

- Hepatectomy consists of systematic resection and partial (non-systematic) resection.

- Indication criteria for hepatectomy

(1) The patient is capable of tolerating surgery

(2) The primary tumor has been controlled or can be controlled.

(3) The metastatic liver tumor can be completely resected.

(4) There are no extrahepatic metastases or they can be controlled.

(5) The function of the remaining liver will be adequate.

- Systemic therapy is considered for patients with unresectable liver metastases whose general condition can be maintained at a certain level or higher (PS 0 to PS 2).

- Thermal ablation therapy consists of microwave ablation therapy (MCT) and radiofrequency ablation (RFA).

- If the patient's general condition is poor (PS $\geq 3$ ), or there is no effective chemotherapy, best supportive care (BSC) is provided.

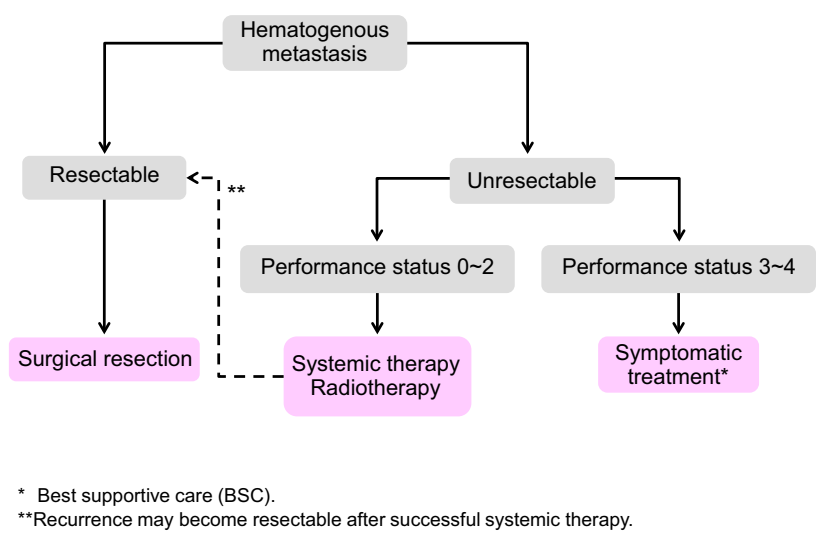

Fig. 5 Treatment strategies for hematogenous metastases 
Comments

[Hepatectomy]

(1) The efficacy of hepatectomy is not based on evidence derived from a cohort study or a randomized controlled trial. However, good results have been shown that were not obtained with other treatments.

(2) The 5-year survival rate after hepatectomy is $35-58 \%$ [74-77]. In a multicenter study conducted in Japan, the 3 -year survival rate of the 585 patients who received hepatectomy was $52.8 \%$ and the 5-year survival rate was $39.2 \%$ [78].

(3) Whether or not the complete resection of the metastatic lesion is possible is determined based on the comprehensive assessment of the number, size, location of metastatic lesions and the predicted residual liver volume.

(4) The sensitivity of magnetic resonance imaging (MRI) in detecting lesions of $<10 \mathrm{~mm}$ in size has been reported to be significantly higher than CT [79]. The efficacy of positron emission tomography (FDG-PET) in the diagnosis and treatment of liver metastasis is not established [80].

(5) Excision without exposure of the cancer to the resected stump is important [81-84].

- There are reports that recommend a resection margin distance of $1 \mathrm{~cm}$ or more $[85,86]$ and that state that no cancer exposure is sufficient [87-90].

(6) In synchronous liver metastasis, excision of the primary tumor may be performed prior to the excision of liver metastasis, which may be excised after the evaluation of the radicality of the primary tumor. There is no clear conclusion as to the timing of resection of synchronous liver metastasis [91-93].

(7) Since the prognosis in the case of hepatic hilar lymph node metastasis is poor, there is a report that hepatic hilar lymph node metastasis is regarded as a contraindication of hepatectomy [94-96].

- In Japan, the 5-year survival rate after the dissection of hepatic hilar lymph node metastasis is reported to be $12.5 \%$ [78].

(8) There are reports showing the efficacy of hepatectomy in patients who have controllable extrahepatic metastases (mainly lung metastases) in addition to liver metastases [59-61, 97] (CQ-8).

(9) A 5-year survival rate of $21-52 \%$ has been reported in cases involving rehepatectomy for residual liver recurrence. For residual liver recurrence cases, resection should be considered in light of the above-mentioned indications for hepatectomy [84, 98-106].

(10) Evidence to support the efficacy of adjuvant chemotherapy after hepatectomy is not sufficient; however, imple- mentation is recommended in view of the high rate of recurrence (CQ-19).

(11) The safety of preoperative chemotherapy for resectable liver metastases has not been established (CQ-9).

[Treatment methods other than resection]

(1) Systemic therapy is performed for patients with unresectable liver metastases.

(2) In cases of inoperable liver metastasis, hepatic arterial infusion therapy or thermal ablation therapy is not generally recommended (CQ-13, CQ-24).

(3) In Japan, there are no data to support the efficacy of stereotactic body radiation therapy or brachytherapy.

(4) If the patient's general condition is poor, an appropriate BSC is provided.

2. Treatment strategies for lung metastases

- Treatment of lung metastases consists of pneumonectomy and systemic therapy, and radiotherapy.

- Pneumonectomy is considered if the metastatic lung tumor is resectable.

- Pneumonectomy consists of systematic resection and partial (non-systematic) resection.

Indication criteria for pneumonectomy

(1) The patient is capable of tolerating surgery.

(2) The primary tumor has been controlled or can be controlled.

(3) The metastatic lung tumor can be completely resected.

(4) There are no extrapulmonary metastases or they can be controlled.

(5) The function of the remaining lung will be adequate.

- Systemic chemotherapy is considered for patients with unresectable lung metastases whose general condition can be maintained at a certain level or higher.

- Even if the patient cannot tolerate surgery, stereotactic body radiation therapy is considered if the primary tumor and extrapulmonary metastases are controlled or can be controlled and the number of lung metastases within $5 \mathrm{~cm}$ in diameter is no more than three [107].

- If the patient's general condition is poor, an appropriate $\mathrm{BSC}$ is provided. 
Comments

\section{[Pneumonectomy]}

(1) The efficacy of lung resection is not based on evidence derived from a cohort study or randomized controlled trial. However, good results that have not been obtained with other treatments have been shown in appropriately selected patients [97, 108-115].

(2) The 5-year survival rate after pulmonary resection is 30-68\% [116-118]. In the multicenter aggregate conducted in the JSCCR project study, the 5-year survival rate of lung resection cases was $46.7 \%$ and the cumulative 5-year relapse-free survival rate was $33.7 \%$, while the 5-year survival rate of non-resected cases was $3.9 \%$ [116, 119].

(3) In synchronous pulmonary metastasis, it is desirable to initially resect the primary lesion and evaluate local curability. Thus, in principle, metachronous resection is performed for synchronous pulmonary metastasis.

(4) The number, size, location, and intra-bronchial development of metastatic lesions should be evaluated, and a procedure that enables the complete resection of the metastatic lesion with secure resection margins should be decided.

(5) The significance of hilar/mediastinal lymph node dissection is not established. The number of metastases, bilateral lung metastasis, hilar/mediastinal lymph node metastasis, serum CEA value before lung resection, primary factor ( $\mathrm{T}$ factor, $\mathrm{N}$ factor), and disease-free interval (DFI) is reported to be poor prognostic factors [112-116, 120].

(6) In cases of controllable extrapulmonary metastasis (mainly liver metastasis), there are reports suggesting the efficacy of lung resection $[60,97,113,115,121$, 122].

(7) A five-year survival rate of $20-48 \%$ has been reported in patients who undergo repeat lung resection for residual lung recurrence $[112,114,115,123,124]$. Even for residual lung recurrence after lung resection, the indications for resection should be carefully considered according to the above-mentioned indication criteria for lung resection.

(8) No large-scale studies have examined the efficacy of adjuvant chemotherapy after the curative resection of lung metastases (CQ-19).

\section{Treatment strategies for brain metastases}

- Brain metastases are often detected as a part of a systemic disease, and surgical therapy or radiotherapy is considered for lesions in which treatment can be expected to be effective.
- The optimal treatment method is selected after considering the patient's general condition and status of other metastatic tumors, and evaluating the size and location of metastatic brain tumors and the number of brain lesions.

- Radiotherapy is considered for patients with unresectable metastases.

[Surgical therapy]

Indications criteria for brain resection [125, 126]

(1) The patient is capable of tolerating surgery.

(2) The primary tumor has been controlled or can be controlled.

(3) The patient has a life expectancy of at least several months.

(4) Resection will not cause significant neurologic symptoms.

(5) There are no metastases to other organs or they can be controlled.

[Radiotherapy]

- The purpose of radiotherapy is to relieve symptoms, such as cranial nerve symptoms and intracranial hypertension symptoms, and to prolong survival time by reducing locoregional relapse.

- Whole-brain radiotherapy is considered for patients with multiple brain metastases and for patients with a solitary brain metastasis for which surgical resection is not indicated.

- Stereotactic irradiation is considered when the number of brain metastases is about no more than three or four and the maximum diameter of each metastasis does not exceed $3 \mathrm{~cm}$.

\section{Comments}

[Surgical therapy]

(1) Approximately, $90 \%$ of cases of brain metastasis involve metastasis to other organs, and the prognosis is poor, even if resection is performed [125, 127-131].

(2) The average survival time after excision of solitary brain metastasis is reported to be $30-40$ weeks [125, 126, 128, $129,132]$. However, the efficacy of surgical therapy has not been determined based on the evaluation of a suitably sized cohort.

(3) The significance of adding whole-brain radiotherapy after brain metastasis resection is controversial [125]. 
[Radiotherapy]

(1) The symptom improvement rate is $60-80 \%[133,134]$.

(2) Stereotactic irradiation achieves a local control rate of 80-90\% [135].

(3) According to a systematic review, the median survival time after stereotactic irradiation, whole-brain radiotherapy, and BSC was 6.4 months, 4.4 months, and 1.8 months, respectively [136].

(4) Age, PS, number of brain metastases, and control of extracranial lesions have been reported as prognostic factors [137-139].

(5) At present, whole-brain radiotherapy is performed irrespective of the number of metastases. When a prognosis of several years can be expected, whole-brain radiotherapy in combination with stereotactic irradiation is considered [140, 141]. In the case of stereotactic irradiation, single treatment is also considered as a treatment option as it can achieve a high QOL. However, surveillance at appropriate intervals by image inspection is necessary because the rate of intracranial recurrence is higher in comparison to that after whole-brain radiotherapy.

4. Treatment strategies for hematogenous metastases to other organs

- Resection is also considered for other hematogenous metastases, such as to the adrenal glands, skin, and spleen, if they are resectable. However, patients with such metastases often have metastasis to more than one organ, and chemotherapy or radiotherapy is often indicated.

Chapter 5: Systemic therapy

- Systemic therapy consists of adjuvant chemotherapy to prevent postoperative recurrence and systemic therapy to treat unresectable colorectal cancer.

- Commonly used anticancer drugs that have been approved for the indication of colorectal cancer and are covered by the Japanese National Health Insurance include the followings:

Cytotoxic drugs: fluorouracil (5-FU), 5-FU + levofolinate calcium $(l-\mathrm{LV})$, tegafur uracil (UFT), tegafur gimeracil oteracil potassium (S-1), UFT + calcium folinate (LV), capecitabine (Cape), irinotecan hydrochloride hydrate (IRI), oxaliplatin (OX), trifluridine/tipiracil hydrochloride (FTD/ TPI), etc.

Molecular targeted drugs: bevacizumab (BEV), ramucirumab (RAM), aflibercept beta (AFL), cetuximab (CET), panitumumab (PANI), regorafenib hydrate (REG)

Immune checkpoint inhibitor: pembrolizumab (Pembro)
1. Adjuvant chemotherapy

- Postoperative adjuvant chemotherapy is a systemic chemotherapy that is performed after surgery to prevent recurrence and improve the prognosis of patients who have undergone $\mathrm{R} 0$ resection.

General principles for the indications of adjuvant chemotherapy

(1) Stage III colorectal cancer (colon and rectal cancer) for which R0 resection has been performed.

(2) The patient has recovered from postoperative complications, if any.

(3) Performance status (PS) of 0 or 1.

(4) The function of major organs is maintained

(5) The patient has no serious complications (particularly bowel obstruction, diarrhea or fever).

* For age, see CQ-17.

- For patients who have Stage II colorectal cancer with a high risk of recurrence, the indications for adjuvant chemotherapy are considered (CQ-18).

- For Stage IV resection cases, see CQ-19.

\section{Recommended therapies (CQ-15)}

The postoperative adjuvant chemotherapy regimens that were shown to be useful in clinical trials and which are covered by the Japanese National Health Insurance program are as follows:

\begin{tabular}{ll}
\hline Oxaliplatin (OX) combination therapy & CAPOX $(\text { Preferred })^{*}$ \\
& FOLFOX $(\text { Preferred })^{*}$ \\
\hline Fluoropyrimidine (FP) monotherapy & Cape \\
& $5-\mathrm{FU}+l-\mathrm{LV}$ \\
& $\mathrm{UFT}+\mathrm{LV}$ \\
& $\mathrm{S}-1$ \\
\hline
\end{tabular}

*See CQ-15.

Recommended administration period (CQ-16)

- In principle, the administration period is 6 months.

Comments

(1) Postoperative adjuvant chemotherapy and treatment regimens are determined with appropriate informed consent, taking into consideration the expected reduction in the risk of recurrence, which is determined based on 
the tumor characteristics (pathological stage, histological type, primary tumor location, biomarkers), treatment characteristics (adverse events, quality of life, treatment costs, etc.), and patient characteristics (age, comorbidities, preferences for assumed side effects, willingness to undergo treatment). Postoperative adjuvant chemotherapy should be started within approximately 8 weeks after surgery.

(2) In an integrated analysis of three randomized controlled trials (RCT) for patients with Dukes' B and Dukes' $\mathrm{C}$ colon cancer, 5 -FU +1 - $\mathrm{LV}$ was associated with an increase in overall survival in comparison to surgery alone. In addition, OX combination therapy for patients with Stage III colon cancer was associated with a significant reduction in the risk of recurrence and an improved prognosis in comparison to $5-\mathrm{FU}+1-\mathrm{LV}$ in 3 RCTs conducted in Europe and the United States $[142,143]$. UFT + LV and Cape showed non-inferiority to $5-\mathrm{FU}+1$ - $\mathrm{LV}$ [144]. S-1 showed non-inferiority to $\mathrm{UFT}+\mathrm{LV}$ [145]. On the other hand, the non-inferiority of S-1 to Cape was not shown [146] (CQ-15).

(3) The administration period of OX combination therapy in postoperative adjuvant chemotherapy for patients with Stage III colon cancer was compared in an integrated analysis of 6 RCTs, including a Japanese RCT (ACHIEVE trial). In the 3-month treatment group, non-inferiority to the 6-month administration group was not shown in any subjects (IDEA collaboration) [147]. However, in the case of CAPOX, the suppression of recurrence in the 3-month administration group was demonstrated to be comparable to that of the 6-month administration group, especially in cases with a low risk of recurrence. In the ACHIEVE trial, the 3-year disease-free survival rates of the 3-month and 6-month administration groups were also similar [148]. The incidence of sensory peripheral neuropathy was significantly lower in the 3-month administration group [149]. On the other hand, with the postoperative adjuvant chemotherapy for colon cancer in Stage IIB/III (TNM-6 edition), 18 months administration of UFT + LV did not demonstrate superiority to six months administration [150]. Furthermore, 12 months administration of Cape for colon cancer in Stage III did not show superiority in the disease-free survival to six months administration [151] (CQ-16).

(4) Although there is less evidence to support postoperative adjuvant chemotherapy for rectal cancer than there is for colon cancer, the efficacy is almost the same as that in colon cancer. Thus, it can be carried out with reference to the evidence of colon cancer. In postoperative UFT alone (1 year) for Stage III rectal cancer (including the anal canal), significant suppression of recurrence and a survival benefit in comparison to surgery alone were observed in a Japanese RCT [152]. Thereafter, S-1 (1 year) showed a significant relapse-suppressing effect in comparison to UFT alone (1 year) for Stage II/III rectal cancer (including the anal canal and excluding RS) [153] (CQ-15).

(5) In an RCT of postoperative adjuvant chemotherapy in Japan for patients with Stage II colon cancer, UFT alone (1 year) did not prevent cancer recurrence in comparison to surgery alone [154] (CQ-18).

(6) In postoperative adjuvant chemotherapy for patients with Stage II/III colon cancer, the concomitant use of IRI or molecular targeted drugs is not recommended.

(7) UFT + LV showed significantly better relapse-free survival in comparison to surgery alone in an RCT of adjuvant chemotherapy for patients after curative resection of liver metastasis in Japan [155] (CQ-19).

2. Systemic therapy for unresectable colorectal cancer (Figs. 6 and 7)

- In the BSC without any systemic therapy, the median survival time (MST) of patients with unresectable colorectal cancer has been reported to be approximately 8 months [156]. Although recent systemic therapy has extended MST to approximately 30 months [157-159], unresectable colorectal cancer remains difficult to cure.

- The purpose of systemic therapy is to prolong survival time and control symptoms by delaying tumor progression. Initially, unresectable colorectal cancer may become resectable after successful systemic therapy.

- Randomized controlled trials involving PS 0-2 patients have shown that systemic therapy is associated with a significantly longer survival time than BSC without any systemic therapy $[156,160]$.

- When considering systemic therapy, it should first be decided whether or not it is applicable. [The steps in the decision-making process for first-line treatment in unresectable colorectal cancer (Fig. 6)].

- Patients appropriate for intensive systemic therapy (fit) include those with no serious comorbidities who are considered tolerant to first-line therapy with $\mathrm{OX}$ and IRI, as well as concomitant therapy with molecular targeted drugs (Fig. 6).

- Patients inappropriate for intensive systemic therapy (vulnerable) include those with some comorbidities who are considered intolerant to first-line therapy with OX and IRI, as well as concomitant therapy with molecular targeted drugs.

- Patients inappropriate for systemic therapy (frail) include those with serious comorbidities who are considered intolerant to first-line therapy. 


\section{Steps in decision-making process for the first-line treatment in unresectable colorectal cancer}

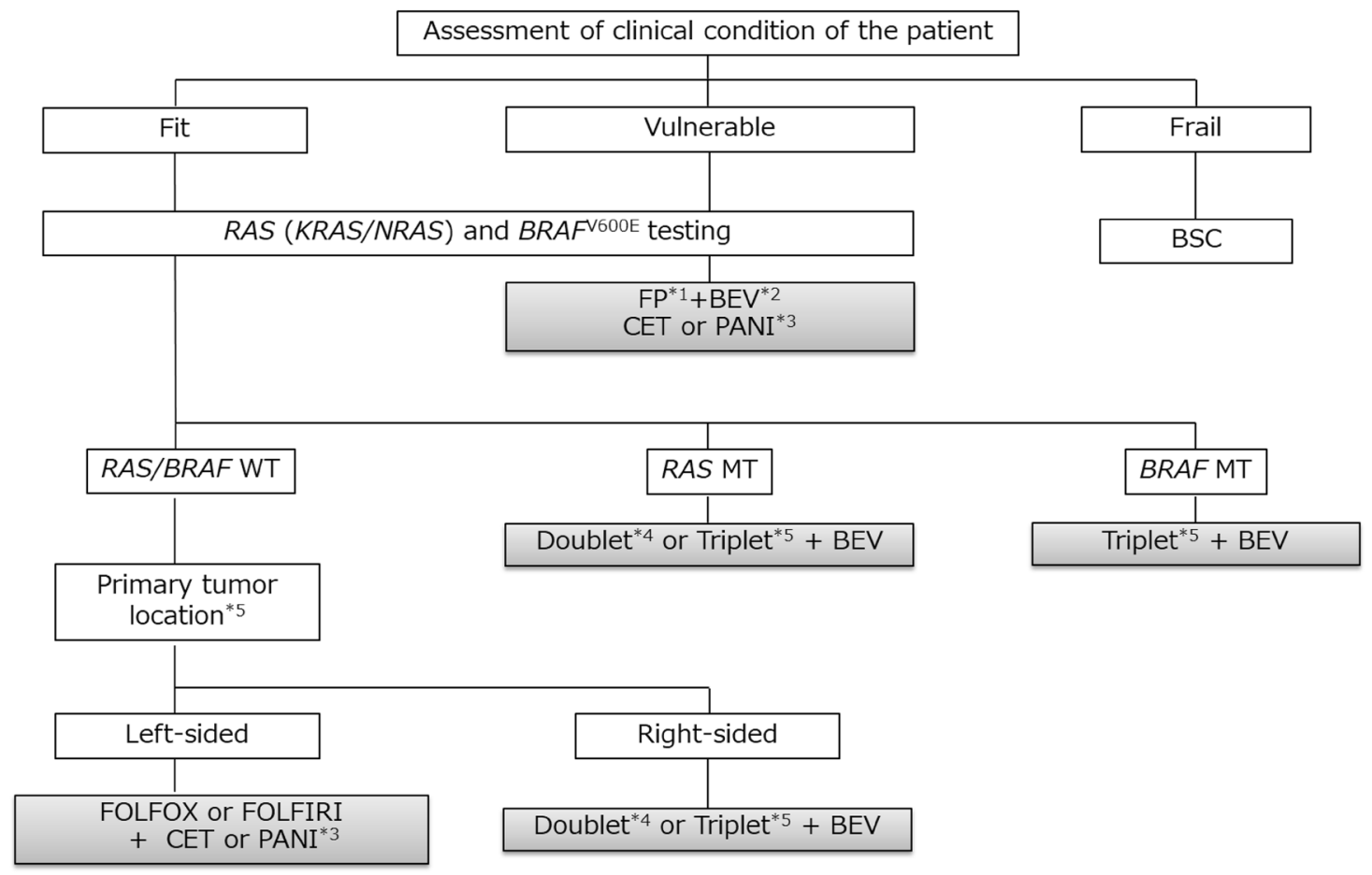

BEV : Bevacizumab, CET : Cetuximab, PANI : Panitumumab, FP : Fluoropyrimidine, WT: wild-type MT: mutant

*1: 5-FU+l-LV, UFT+LV, S-1, Cape

$* 2$ : Combination therapy with BEV is recommended. If not applicable, FP monotherapy is acceptable.

*3: CET and PANI are applicable for patients with RAS (KRAS/NRAS) WT only.

*4 : Doublet : FOLFOX, CAPOX, SOX, FOLFIRI, S-1+IRI

$* 5$ : Triplet : FOLFOXIRI

*6 : Cancers proximal to the splenic flexure (ie, tumors occurring in the cecum, ascending colon, or transverse colon) were classified as right-sided. Left-sided cancers included those distal to the splenic flexure (ie, tumors occurring in the descending colon, sigmoid colon, or rectum).

Fig. 6 Steps in the decision-making process for the first-line treatment in unresectable colorectal cancer

- $R A S(K R A S / N R A S)$ mutation testing and $B R A F^{\mathrm{V} 600 \mathrm{E}}$ mutation testing should be performed before first-line therapy for patients appropriate for systemic therapy.

- CET and PANI are only indicated for patients with wild-type RAS (KRAS/NRAS) (Comment \& [161]).

- Pembro is only indicated for patients with high-frequency microsatellite instability (MSI-H) (CQ-23).

General principles underlying the indications of systemic therapy

(1) The clinical or histopathological diagnosis has been confirmed as colorectal cancer.

(2) The curative resection is not possible.
(3) Patients are defined as "fit" or "vulnerable" depending on the general condition, the main organ function, and the presence or absence of serious comorbidities (refer to the package insert of each drug).

- The efficacy and safety of the following regimens have been confirmed in clinical trials. These systemic therapies are approved and covered by the Japanese National Health Insurance system.

First-line therapy (CQ-20)

The following regimens are considered as systemic therapy for first-line therapy.

FOLFOX $^{\text {note } 1}[162,163] \pm$ BEV [164]

CAPOX + BEV [164, 165]

SOX+BEV [157] 
Fig. 7 Systemic therapy algorithm for unresectable colorectal cancer

\section{Systemic therapy algorithm for unresectable colorectal cancer}

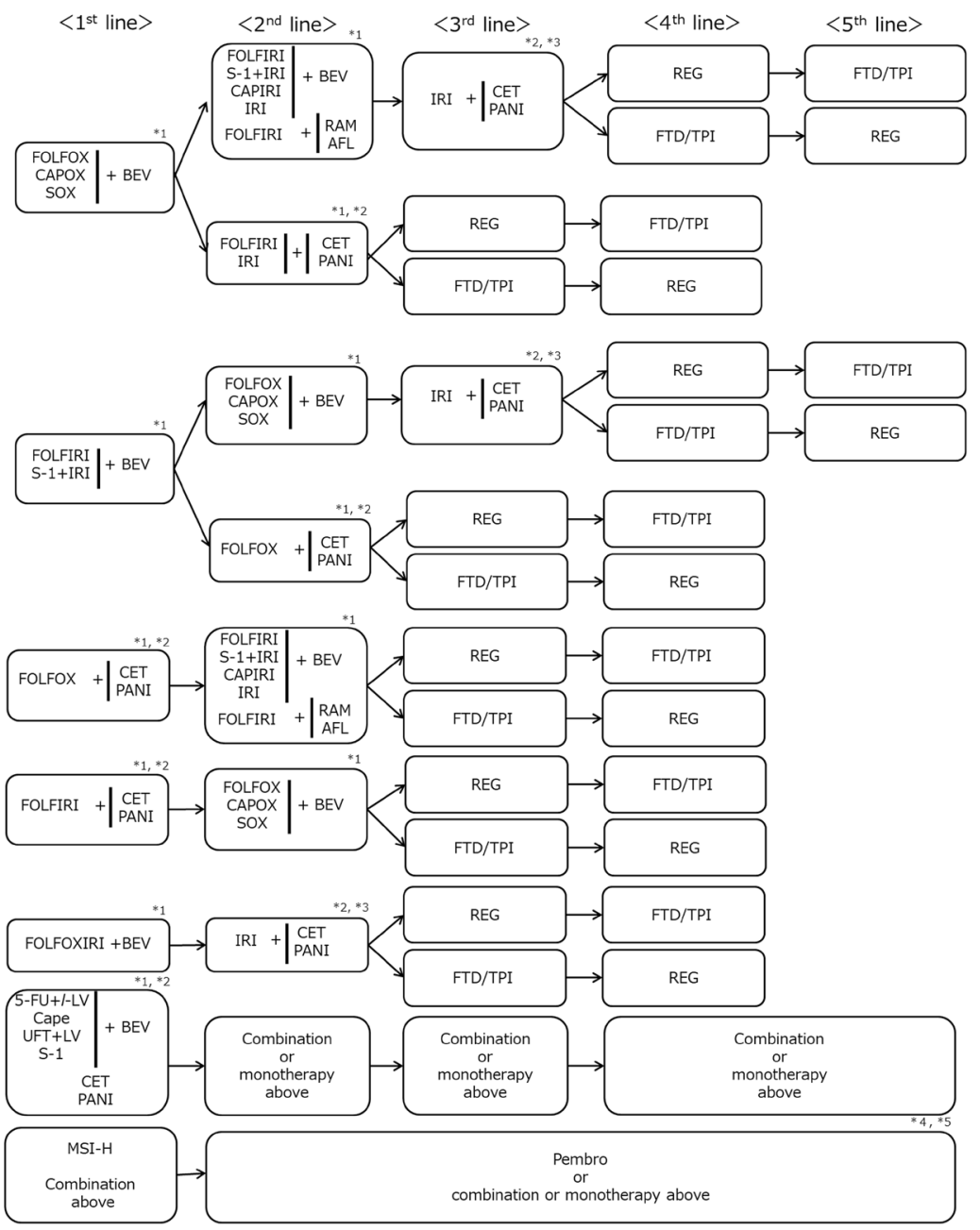

BEV : bevacizumab, RAM : ramucirumab, AFL: aflibercept beta, CET : cetuximab, PANI : panitumumab, REG : regorafenib, FTD/TPI : trifluridine/tipiracil hydrochloride, Pembro : pembrolizumab

* 1 : Combination with molecular targeted drugs such as BEV, RAM, AFL, CET, PANI is recommended, but for patients who are not candidates, chemotherapy alone is acceptable.

*2 : Indicated to RAS wild-type only.

$* 3$ : If possible, combined with IRI is recommended.

*4 : Indicated to MSI-H only.

* 5 : Pembro is also considered for the patients without candidate for chemotherapy or other molecular targeted drugs due to patient condition or comorbidities. Package inserts and Optimal Clinical Use Guidelines should be referred to when using Pembro.
FOLFIRI $[166,167]+$ BEV $[159,168]$

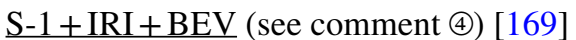

FOLFOX + CET [170], FOLFOX + PANI [171]

FOLFIRI + CET [172], FOLFIRI + PANI [173]

FOLFOXIRI [174] \pm BEV $[158,175]$

Infusional 5-FU $+l-\mathrm{LV}[176,177]+\mathrm{BEV}[178,179]$

Cape $[180,181]+$ BEV [182]

UFT + LV [183-185] + BEV [186]

S-1 + BEV [187]

CET or PANI $[188,189]$
Second-line therapy (CQ-21)

The following regimens are considered as systemic therapy for second-line therapy.

(a) For patients who are refractory or intolerant to the firstline therapy, including OX.

FOLFIRI + BEV $[190,191]$

CAPIRI + BEV (see comment (5) [192]

FOLFIRI+RAM [193] 
FOLFIRI+AFL (see comment (6) [194]

$\underline{\mathrm{S}-1+\mathrm{IRI}}[195]+\mathrm{BEV}$

IRI [196] + BEV [197]

FOLFIRI (or IRI) + CET, FOLFIRI + PANI [198, 199]

IRI + CET [200] or IRI +

Pembro (see comment (10) [202]

(b) For patients who are refractory or intolerant to the firstline therapy, including IRI

FOLFOX [203]+BEV [190, 204]

CAPOX [205]+ BEV [190]

$\mathrm{SOX}+\mathrm{BEV}$

FOLFOX + CET, FOLFOX + PANI

Pembro (see comment (10) [202]

(c) For patients who are refractory or intolerant to the firstline therapy, including 5-FU, OX and IRI

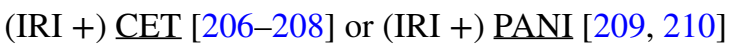

Pembro (see comment (10) [202]

Third-line and subsequent therapies (CQ-22)

The following regimens are considered as systemic therapy for third-line and subsequent therapies

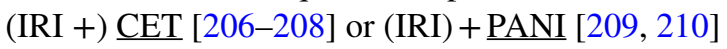

REG [211]

FTD/TPI [212-214]

Pembro (see comment (10) [202]

Note 1 The efficacy and safety of the underlined regimen have been validated in Phase III trials.

Comments

(1) When administering OX, it is necessary to pay attention to the cumulative neurotoxicity of OX. Although Grade 2 neurotoxicity which impairs tolerability was observed, if the curative effect persists, stopping OX and switching to fluoropyrimidine \pm BEV/CET/PANI, etc., should be considered. If the disease progress and neurotoxicity is improved to Grade 1 or less, reintroduction of OX should be considered.

(2) Careful attention is required when using IRI for patients with constitutional jaundice, such as that caused by Gilbert's syndrome, or those with high serum bilirubin or who are in a poor general condition (PS 2). Associations between genetic polymorphisms of enzymes that metabolize IRI (UGTIAI) and toxicity have been suggested. Although the maximum tolerated dose of IRI was confirmed to be $150 \mathrm{mg} / \mathrm{m} 2$ for patients with UGT1A1 homozygous $(* 28 / * 28, * 6 / * 6$, or $* 28 / * 6)$, grade 3 or higher neutropenia was observed in $62.5 \%$ of these patients during the first cycle [215].

(3) In Japan, the efficacy and safety of FOLFOXIRI+ BEV were confirmed in the QUATTRO trial* [216]. This trial enrolled the patients of 20-75 years of age, with PS 0-1 (PS 0: 71-75 years of age), and without UGTIAI homozygous $(* 28 / * 28, * 6 / * 6$, or $* 28 / * 6)$. Thus, the efficacy and safety were not confirmed among other patients. In this study, grade 3/4 neutropenia and febrile neutropenia were observed in $72.5 \%$ and $21.7 \%$ of patients, respectively. Grade 4 neutropenia and febrile neutropenia during the early cycles were higher in patients heterozygous for UGTIAl $(* 28 / * 1, * 6 / * 1)$ in comparison to wild type $(* 1 / * 1)$.

*The QUATTRO trial, a phase II trial conducted in Japan, confirmed the efficacy and safety of FOLFOXIRI + BEV in first-line therapy for patients with unresectable colorectal cancer.

(4) The efficacy and safety of S-1 + IRI + BEV were confirmed in the TRICOLORE trial [169]. The TRICOLORE trial, a phase III trial conducted in Japan, compared the efficacy and safety of S-1 + IRI + BEV with FOLFOX + BEV or CAPOX + BEV as first-line therapy for PS 0-1 patients with unresectable colorectal cancer.

(5) The efficacy and safety of CAPIRI + BEV were confirmed in the AXEPT trial* [192].

* The AXEPT trial, a phase III trial conducted in Asia, compared the efficacy and safety of CAPIRI \pm BEV with FOLFIRI \pm BEV as second-line therapy for PS 0-2 patients with unresectable colorectal cancer.

(6) The efficacy and safety of AFL were confirmed in the VELOUR trial* [194]. The administration of AFL is approved in combination with 5-FU, 1 -LV, and IRI in Japan. As described in the package insert, the efficacy and safety of AFL in first-line therapy have not been established.

*The VELOUR trial, an international cooperative phase III trial, compared the efficacy and safety of FOLFIRI + AFL with FOLFIRI + placebo as secondline therapy for PS 0-2 patients with unresectable colorectal cancer who were refractory or intolerant to prior combination therapy with fluoropyrimidine and OX.

(7) Although hepatic arterial infusion therapy is associated with high response rates in patients with liver metastasis, it does not show any survival benefit in comparison to systemic therapy [217] (CQ-24). 
(8) RAS (KRAS/NRAS) gene mutations are detected in approximately $50 \%$ of patients with unresectable colorectal cancer, and it was reported that the efficacy of anti-EGFR antibody therapy (CET, PANI) cannot be expected for patients with these mutations. Thus, it is recommended that RAS (KRAS/NRAS) mutation testing should be performed prior to first-line therapy for patients who can receive systemic therapy [218, 219] (CQ-20). In Japan, RAS (KRAS/NRAS) mutation testing has been reimbursed since April 2015. In a recent pooled analysis that included six randomized trials comparing chemotherapy plus anti-EGFR antibody therapy with chemotherapy or chemotherapy plus BEV, chemotherapy plus anti-EGFR antibody therapy showed superior efficacy in patients with left-sided tumors (descending colon, sigmoid colon, rectum) in comparison to those with right-sided tumors (cecum, ascending colon, transverse colon) [220].

(9) In Japan, $B R A F^{\mathrm{V} 600 \mathrm{E}}$ gene mutations are detected in approximately $5 \%$ of patients with unresectable colorectal cancer, and patients with these mutations are resistant to systemic therapy and have a very poor prognosis [221, 222]. Based on a subgroup analysis in the TRIBE trial, FOLFOXIRI + BEV therapy might be effective as a first-line therapy for patients with $B R A F^{\mathrm{V} 600 \mathrm{E}}$ gene mutations [223]. Thus, it is recommended that $B R A F^{\mathrm{V} 600 \mathrm{E}}$ mutation testing should be performed prior to the administration of first-line therapy for patients who can receive systemic therapy [219, 220] (CQ-20). $B R A F^{\mathrm{V} 600 \mathrm{E}}$ mutation testing is also useful as an adjunct diagnostic test for Lynch syndrome. Thus, this test is recommended for patients with DNA mismatch repair deficiency and suspected Lynch syndrome. For the basic requirements of the $B R A F^{\mathrm{V} 600 \mathrm{E}}$ mutation testing, refer to "Japanese Society of Medical Oncology (JSMO) Clinical Guidelines: Molecular testing for Colorectal Cancer Treatment, Third Edition" [220]. In Japan, $B R A F^{\mathrm{V} 600 \mathrm{E}}$ mutation testing was reimbursed since August 2018. Recently, the efficacy of combination therapy of BRAF inhibitors and anti-EGFR antibodies for patients with $B R A F^{\mathrm{V} 600 \mathrm{E}}$ gene mutation was reported [224, 225]. In the NCCN guideline version 1.2018, combination therapy of IRI + anti-EGFR antibody + Vemurafenib (BRAF inhibitor) is listed as a recommended regimen in second or later line for these patients (unapproved in Japan as of January 2019).

(10) MMR (mismatch repair) deficiency was mainly observed in patients with colorectal cancer associated with Lynch syndrome caused by germline mutations of genes associated with MMR or sporadic colorectal cancer caused by acquired $M L H 1$ gene methylation. Tests for tumor MMR deficiency include MSI testing and immunohistochemistry (IHC) for MMR proteins. According to Western data,
MSI-H is recognized in approximately $5 \%$ of unresectable colorectal cancer (approximately $2-3 \%$ in Japan). There is no established systemic therapy specifically for unresectable colorectal cancer with MMR deficiency. Thus, under the current circumstances, the common regimens for sporadic colorectal cancers are indicated for these patients. Recently, the efficacy of anti-PD-1 antibody therapies (pembrolizumab [Pembro] and nivolumab) against unresectable colorectal cancer with MMR deficiency was reported. In the United States, these therapies are approved for unresectable colorectal cancer with MMR deficiency [202, 226]. In Japan, Pembro was approved for MSI-H solid cancer (only for patients for whom standard systemic therapy is not appropriate), including MSI-H unresectable colorectal cancer, in December 2018 (CQ-23). At the same time, the MSI testing Kit (FALCO) was reimbursed as a companion diagnostic test. MSI testing can also be used in screening for Lynch syndrome. Thus, it is recommended that physicians refer to the "JSCCR Guidelines 2016 for the Clinical Practice of Hereditary Colorectal Cancer" [227] for the explanation of the test, the interpretation of the results, and correspondence in cases of suspected Lynch syndrome. It is reported that the concordance rate between the MSI testing and IHC for MMR protein is high in colorectal cancer.

\section{Chapter 6: Radiotherapy}

- Radiotherapy is used to treat patients with locally advanced rectal cancer either as adjuvant therapy after surgery to prevent recurrence or before surgery to reduce tumor volume and preserve the anal sphincter, and also as palliative care to relieve the symptoms and prolong the survival time of patients with unresectable colorectal cancer who have symptomatic lesions.

\section{Adjuvant radiotherapy}

- Adjuvant radiotherapy is classified into three categories, according to the timing of surgery and radiation therapy: preoperative radiotherapy, intraoperative radiotherapy, and postoperative radiotherapy.

- The purpose of adjuvant radiotherapy is to improve the local control rate and the survival rate of rectal cancer patients. The purpose of preoperative radiotherapy includes improving the anal sphincter preservation rate and improving the resection rate. However, insufficient evidence of improved survival has been found to make this the objective of adjuvant radiotherapy.

- Preoperative radiotherapy is indicated for patients with $\mathrm{T}$ stage clinically diagnosed as "invasion depth cT3 or 
deeper or cN-positive"; postoperative radiotherapy is indicated for patients with $\mathrm{T}$ stage pathologically diagnosed after surgery as "invasion depth pT3 or deeper or $\mathrm{pN}$ positive, where the existence of a surgical dissection plane positive (RM1) or penetration of the surgical dissection plane by the cancer (RMX) is unclear"; and intraoperative radiotherapy is indicated for "surgical dissection plane positive (RM1) or penetration of the surgical dissection plane by the cancer (RMX) is unclear".

- Radiotherapy is delivered with a linear accelerator, with electron beams being used for intraoperative radiotherapy and photon beams for external radiotherapy.

\section{Comments}

(1) Preoperative radiotherapy (CQ-25)

1. Preoperative radiotherapy has the following advantages: seeding during surgery can be prevented by inactivating lesions with irradiation; a high percentage of tumor cells are normo-oxic and radiosensitive, because blood flow to the tumor is maintained; there has been little damage to the digestive tract, since the small bowel is not fixed within the pelvic cavity, thereby resulting in low radiation-induced delayed toxicity, which means a less toxic postoperative setting; improvement in the $\mathrm{R} 0$ resection rate and anal sphincter preservation can be expected because of tumor size reduction [228].

2. Preoperative radiotherapy has the following disadvantages: early-stage patients may be subjected to overtreatment and postoperative complications may increase.

3. Twelve phase III clinical trials of preoperative radiotherapy (without chemotherapy) have been reported [228], and in 5 of the 12 randomized controlled trials the local control rate in the group that received preoperative radiotherapy was significantly higher than in the surgery-alone group. However, an improvement in the survival rate was observed in only 1 trial [229].

4. Two meta-analyses of radiotherapy showed improvement in the local control rate compared to surgery alone, and improvement in the survival rate in the groups that received doses of $30 \mathrm{~Gy}$ or more. However, there is controversy as to whether there is improvement in the survival rate $[230,231]$.
5. Trials of short-course radiotherapy with 5 Gy per fraction have been conducted, mainly in Europe $[229,232]$. Because the late effects of radiation depend on the fraction size, long-term follow-up for late adverse effects, such as anal dysfunction and bowel dysfunction, is necessary.

6. In the Dutch CKVO 95-04 trial, which compared preoperative radiotherapy ( 25 Gy delivered in five fractions in one week) + TME and TME alone to investigate the significance of adding short-course radiotherapy to TME, the 5-year and 10-year local control rates were significantly higher in the combination therapy group, but there was no significant difference between the two groups in the 5-year and 10-year survival rates [138, 232, 233]. The incidences of sexual dysfunction and bowel dysfunction were higher in the preoperative radiation combination therapy group than in the surgery-alone group [234, 235].

7. The effect of preoperative radiotherapy in reducing the size of the primary tumor may enable sphincter preservation. When the purpose of the preoperative radiotherapy is sphincter preservation, it is desirable to perform surgery after allowing an appropriate period for the tumor to decrease in size (6-8 weeks after the completion of radiotherapy) [236].

8. In Europe, four randomized controlled trials, including the EORTC trial, were performed to investigate the usefulness of adding chemotherapy to preoperative radiotherapy. The incidence of acute-phase adverse events was significantly higher in the preoperative chemoradiotherapy groups, but the pathologic complete response rates $(\mathrm{pCR})$ were significantly higher than that in the preoperative radiotherapy alone groups. In two trials, the exception being the short-course radiotherapy trial, the local recurrence rate was significantly lower in the preoperative chemoradiotherapy group, and there was no significant difference between the two groups in terms of sphincter preservation or survival rate [237-240].

9. In a randomized controlled trial that compared preoperative chemoradiotherapy and postoperative chemoradiotherapy, there was no significant difference in the 5-year survival rate, but the local recurrence rate and incidence of grade 3 or higher adverse events were significantly lower in the preoperative chemoradiotherapy group. Among the patients in whom abdominoperineal resection (APR) was considered necessary at the time of enrollment, the percentage of patients in whom sphincter preservation was possible was 
significantly higher in the preoperative chemoradiotherapy group [241].

10. A randomized controlled trial of 5-FU versus Cape combination chemotherapy in the preoperative chemoradiotherapy indicated that the two drugs had the same level of efficacy and safety [242, 243]. NCCN guidelines allow the use of either 5-FU or Cape as standard combination chemotherapy in the preoperative chemoradiotherapy. The indications and use of Cape as an adjuvant therapy for rectal cancer have been approved for use under health insurance in Japan as of August 2016.

11. In randomized controlled trials into the efficacy of adding OX to fluoropyrimidine as a combination chemotherapy in the preoperative chemoradiotherapy, OX increased adverse events in three trials, but demonstrated no efficacy with regard to $\mathrm{pCR}$ ratio, localized control ratio and survival [242, 244-246]; moreover, in one trial, although there was no difference in adverse events and no analysis was done into disease-free survival at the primary endpoint, the $\mathrm{pCR}$ ratio was significantly higher [247].

2. Palliative radiotherapy

(a) Intrapelvic lesions (CQ-26)

- The purpose of palliative radiotherapy for intrapelvic lesions is to relieve symptoms such as pain, hemorrhage, and bowel dysfunction caused by intrapelvic tumors.

- The target volume includes the tumor causing the symptoms.

[Dose and fractionation]

- A total dose of 45-50 Gy is administered in 1.82.0 Gy fractions.

- Depending on the patient's general condition, such as performance status, and the severity of the symptoms, radiotherapy may be completed in a shorter term with a larger fraction size, for example $30 \mathrm{~Gy}$ in 10 fractions over 2 weeks.

(b) Extrapelvic lesions

1. Bone metastases
- The purpose of palliative radiotherapy for bone metastases is to achieve pain relief, prevent pathological fractures, and prevent and treat spinal cord paralysis.

- The target volume includes the metastatic bone lesions causing the symptoms.

[Dose and fractionation]

- Local field radiotherapy, such as $30 \mathrm{~Gy}$ in 10 fractions and 20 Gy in 5 fractions, is widely performed.

\section{Brain metastases}

- See the section on hematogenous metastases (Chapter 4).

[Dose and fractionation]

- When whole-brain radiotherapy is performed, $30 \mathrm{~Gy}$ in 10 fractions is the standard treatment. If long-term survival is expected, fractionated radiotherapy, such as $37.5 \mathrm{~Gy}$ in 15 fractions and $40 \mathrm{~Gy}$ in 20 fractions, is considered.

- When stereotactic radiosurgery is performed, a peripheral dose of 16-25 Gy is delivered in a single fraction.

\section{Chapter 7: Palliative care}

- Palliative care is a general term for palliative treatment of various mental and physical symptoms related to cancer.

- Palliative care extends from the time the diagnosis of disease is made to the end stage, and different care should be provided depending on the disease stage and symptoms.

- In principle, cancer treatment should be performed under conditions in which symptom relief is achieved [248], and palliative care should be started at the same time as surgical treatment and systemic therapy.

- Palliative care to improve the QOL of patients with endstage colorectal cancer includes:

(1) Pain relief

(2) Surgical treatment

(3) Systemic therapy

(4) Radiotherapy

(5) Counseling for psychiatric symptoms

Chapter 8: Surveillance after surgery for colorectal cancer

1. Surveillance for recurrence after curability A resection of colorectal cancer 
(1) Consideration should be given to periodic endoscopic examination for recurrence at the site of local resection or anastomosis in pStage 0 [pTis cancer] cases. Surveillance for recurrence in other organs is not necessary.

(2) pStage I-pStage III cases should be surveyed for recurrence in the liver, lungs, local area, anastomosis, lymph nodes, peritoneum, etc. The following points should be noted.

- In principle, the duration of surveillance is 5 years after surgery, and the surveillance examinations should be scheduled at shorter intervals during the first 3 years after surgery.

- It should be noted that there is a higher incidence of lung metastasis and local recurrence in rectal cancer than in colon cancer.

- The following is an example of a surveillance schedule after curative resection of Stage I to Stage III colorectal cancer that was designed on the basis of the results of a retrospective investigation of such factors as the common sites and incidence of recurrence, the efficacy of treatment, and the clinical practice in Japan. (Figure 8)

2. Surveillance after curability B resection of colorectal cancer and after resection of recurrent tumors.
(1) The same surveillance method as for Stage III colorectal cancer is used. It should be noted that recurrence and re-recurrence are common in organs previously operated on. It should also be noted that the frequency of relapse after 5 years is relatively high.

(2) In cases allocated curability B due to $\mathrm{R} 1$ resection, close surveillance schedule should be planned for organs in which residual cancer is suspected.

3. Surveillance of metachronous multiple cancer

- Colonoscopy is performed for surveillance of metachronous multiple colorectal cancer.

\section{Comments}

(1) Aim of surveillance

- The aim of surveillance is to improve the patient's prognosis by early detection and treatment of recurrences [249]. Thus, surveillance is conducted for patients who can be treated when recurrence is found [250].

(2) Recurrence rate, sites of recurrence, times of recurrence

- The results of the JSCCR colorectal cancer registry in 2007 are shown in Figs. 9 and 10 and Tables 10, 11,12 , and 13 . The subjects included 5,103 patients who underwent curative resection of colorectal cancer

\begin{tabular}{|c|c|c|c|c|c|c|c|c|c|c|c|c|c|c|c|c|c|c|c|c|}
\hline \multicolumn{3}{|c|}{ Years/months after surgery } & \multicolumn{3}{|c|}{1 year } & \multicolumn{4}{|c|}{2 years } & \multicolumn{4}{|c|}{3 years } & \multicolumn{4}{|c|}{4 years } & \multicolumn{3}{|c|}{5 years } \\
\hline & $3 m$ & 6 & 9 & 12 & 3 & 6 & 9 & 12 & 3 & 6 & 9 & 12 & 3 & 6 & 9 & 12 & 3 & 6 & 9 & 12 \\
\hline \multicolumn{21}{|l|}{ Colon cancer } \\
\hline \multicolumn{21}{|l|}{$\begin{array}{l}\text { Interview and } \\
\text { examination }\end{array}$} \\
\hline \multicolumn{21}{|l|}{ Tumor marker } \\
\hline \multicolumn{21}{|l|}{ Chest CT } \\
\hline \multicolumn{21}{|l|}{ Abdominal CT } \\
\hline \multicolumn{21}{|l|}{ Colonoscopy } \\
\hline \multicolumn{21}{|l|}{ Rectal cancer } \\
\hline \multicolumn{21}{|l|}{$\begin{array}{l}\text { Interview and } \\
\text { examination }\end{array}$} \\
\hline \multicolumn{21}{|l|}{ Tumor marker } \\
\hline \multicolumn{21}{|l|}{$\begin{array}{l}\text { Digital rectal } \\
\text { examination }\end{array}$} \\
\hline Chest CT & & 0 & & $\bullet$ & & $\bullet$ & & - & & $\bullet$ & & 0 & & O & & - & & O & & $\bullet$ \\
\hline $\begin{array}{l}\text { Abdominal and } \\
\text { pelvic CT }\end{array}$ & & 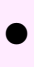 & & 0 & & ○ & & 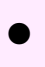 & & 0 & & ? & & O & & $\bullet$ & & O & & $\bullet$ \\
\hline Colonoscopy & & & & ? & & & & 0 & & & & 0 & & & & & & & & \\
\hline
\end{tabular}

-: Performed for Stage I to Stage III colorectal cancer.

O: Performed for Stage III colorectal cancer. Can be omitted in Stage I and Stage II colorectal cancer.

Fig. 8 An example of a surveillance schedule after curative resection of pStage I to pStage III colorectal cancer 


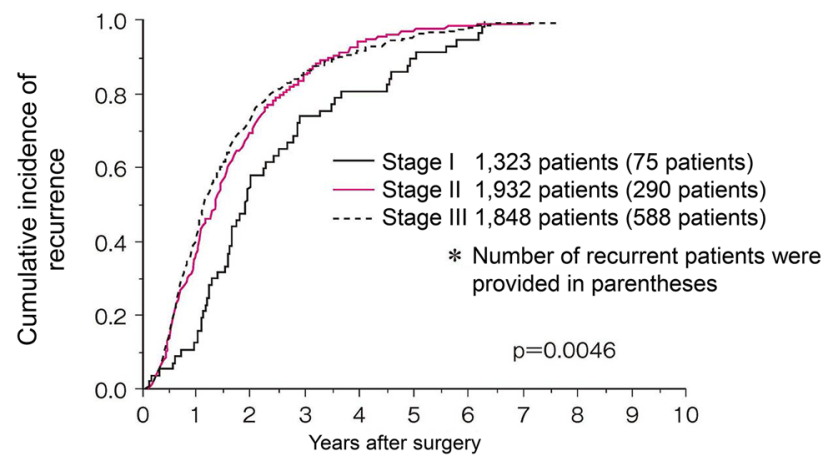

Fig. 9 Cumulative incidence of recurrence according to stage (JSCCR colorectal cancer registry: patients in the year 2007)

in 2007 at the 71 institutions that participated in the registry. The median follow-up period was 6.0 years.

(1) Timing of recurrence and sites of the recurrences (Figs. 9, 10, Tables 10, 12, 13).

- More than $85 \%$ of the recurrences were detected within 3 years after surgery, and more than $95 \%$ of the recurrences were detected within 5 years after surgery.
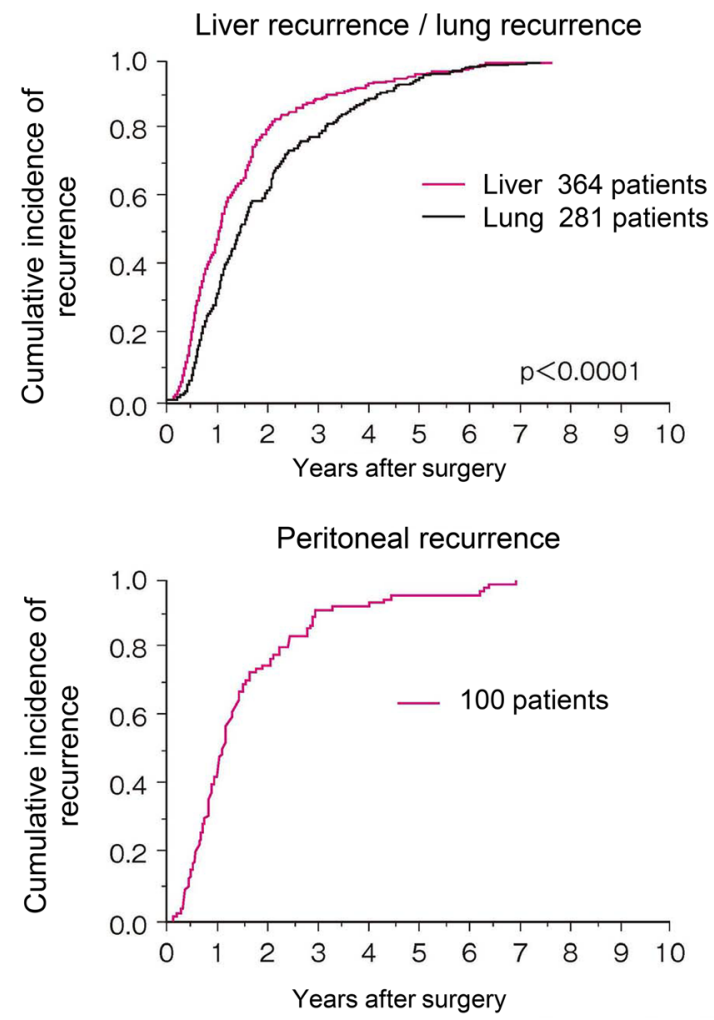

- The overall incidence of recurrence more than 5 years after surgery was less than $1 \%$.

- The appearance of pulmonary recurrence tended to be slower than that of liver metastasis.

- Local recurrence and lung recurrence were more frequent in rectal cancer than in colon cancer. In contrast, peritoneal recurrence was more frequent in colon cancer than in rectal cancer.

(2) Characteristics of recurrence according to pStage (Fig. 9, Tables 10, 11)

1. pStage I

- The recurrence rates of colon cancer and rectal cancer were $4.4 \%$ and $7.4 \%$, respectively. Rectal cancer was associated with a higher rate of recurrence.

- The recurrence rates of pT1 cancer and pT2 cancer were $4.0 \%$, and $7.3 \%$, respectively.

- In pStage I cases, the appearance of recurrence was delayed in comparison to pStage II and pStage III cases, and recurrence appeared after 5 years in more than $8 \%$ of patients with recurrent disease. Among all pStage I cases, the proportion of
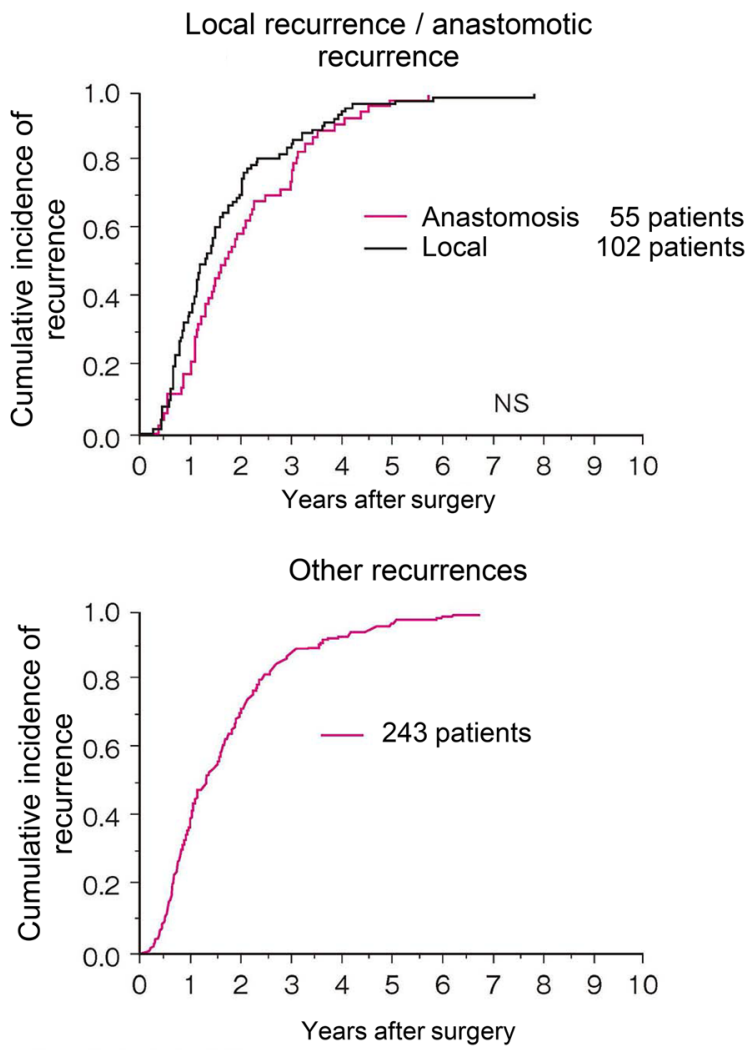

Fig. 10 Cumulative incidence of recurrence according to the site of recurrence (JSCCR colorectal cancer registry: patients in the year 2007) 
Table 10 Recurrence rate after curative resection of colorectal cancer according to pStage and cumulative incidence of recurrence according to the number of years after surgery

\begin{tabular}{llllll}
\hline $\begin{array}{l}\text { pStage (no. of } \begin{array}{l}\text { Recurrence rate (no. of } \\
\text { patients) }\end{array} \\
\text { patients with recurrence) }\end{array}$ & $\begin{array}{l}\text { Cumulative incidence of recurrence according to the } \\
\text { no. of years after surgery (cumulative no. of patients } \\
\text { with recurrence) }\end{array}$ & $\begin{array}{l}\text { Percentage of patients experiencing } \\
\text { recurrence more than 5 years after } \\
\text { surgery among all patients (no. of } \\
\text { patients) }\end{array}$ \\
\cline { 3 - 5 } 3 years & 4 years & 5 years & $0.4 \%$ \\
I & $5.7 \%$ & $73.7 \%$ & $80.7 \%$ & $91.2 \%$ & $(5)$ \\
$(1323)$ & $(75)$ & $(42)$ & $(46)$ & $(52)$ & $0.3 \%$ \\
II & $15.0 \%$ & $86.0 \%$ & $94.2 \%$ & $97.7 \%$ & $(6)$ \\
$(1932)$ & $(290)$ & $(221)$ & $(242)$ & $(251)$ & $1.1 \%$ \\
III & $31.8 \%$ & $86.7 \%$ & $92.0 \%$ & $96.5 \%$ & $(19)$ \\
$(1848)$ & $(588)$ & $(475)$ & $(504)$ & $(529)$ & $0.6 \%$ \\
All & $18.7 \%$ & $85.6 \%$ & $91.9 \%$ & $96.5 \%$ & $(30)$ \\
$(5103)$ & $(953)$ & $(738)$ & $(792)$ & $(832)$ & \\
\hline
\end{tabular}

JSCCR colorectal cancer registry (patients in the year 2007); 91 patients were excluded from analyses for cumulative incidence of recurrence because of unknown recurrence date

Table 11 Recurrence rate of pStage I colorectal cancer

\begin{tabular}{|c|c|c|c|c|}
\hline pStage I & No. of patients & $\begin{array}{l}\text { No. of patients with } \\
\text { recurrence }\end{array}$ & $\begin{array}{l}\text { Recurrence rate } \\
(\%)\end{array}$ & $p$ value \\
\hline \multicolumn{5}{|c|}{ Tumor location } \\
\hline Colon & 756 & 33 & 4.4 & \multirow[t]{2}{*}{$p=0.0186$} \\
\hline Rectum & 567 & 42 & 7.4 & \\
\hline \multicolumn{5}{|c|}{ Depth of tumor invasion } \\
\hline SM & 655 & 26 & 4.0 & \multirow[t]{2}{*}{$p=0.0076$} \\
\hline MP & 668 & 49 & 7.3 & \\
\hline \multicolumn{5}{|c|}{$\begin{array}{l}\text { Tumor location and depth of } \\
\text { tumor invasion }\end{array}$} \\
\hline \multicolumn{5}{|l|}{ Colon } \\
\hline SM & 403 & 10 & 2.5 & \multirow[t]{2}{*}{$p=0.0063$} \\
\hline MP & 353 & 23 & 6.5 & \\
\hline \multicolumn{5}{|l|}{ Rectum } \\
\hline SM & 252 & 16 & 6.4 & \multirow[t]{2}{*}{ NS } \\
\hline MP & 315 & 26 & 8.3 & \\
\hline
\end{tabular}

JSCCR colorectal cancer registry: (patients in the year 2007); RS cancer was counted as rectal cancer patients with recurrence after 5 years was less than $0.5 \%$.

\section{2. pStage II, pStage III}

- The recurrence rates of pStage II and pStage III were $15.0 \%$ and $31.8 \%$, respectively.

- Recurrence was detected within 3 years after surgery in more than $85 \%$ of patients with recurrent disease.

- The incidence of recurrence at more than 5 years after surgery in patients with pStage II and pStage III colorectal cancer was $0.3 \%$ and $1.1 \%$, respectively.

(3) Surveillance for recurrence after curability A resection of colorectal cancer
- The surveillance schedule shown in Fig. 8 was prepared in consideration of the frequency of recurrence for each stage, the site and timing of recurrence, and current surveillance practices in Japan.

- The diagnostic modalities and schedule density differ between the guidelines. The current surveillance methods in Japan are generally intensive in comparison to those adopted in representative guidelines from Western countries (NCCN [251], ESMO [252], ASCO [250, 253], ASCRS [254].

(4) Surveillance of metachronous multiple primary cancers 
Table 12 Recurrence rate according to the site of the first recurrence after curative resection of colorectal cancer and cumulative incidence of recurrence according to the number of years after surgery

\begin{tabular}{|c|c|c|c|c|c|}
\hline \multirow[t]{2}{*}{ Site of first recurrence } & \multirow[t]{2}{*}{$\begin{array}{l}\text { Recurrence rate (no. of patients } \\
\text { with recurrence (including } \\
\text { overlaps) }\end{array}$} & \multicolumn{3}{|c|}{$\begin{array}{l}\text { Cumulative incidence of recurrence according } \\
\text { to the number of years after surgery (cumula- } \\
\text { tive No. of patients with recurrence) }\end{array}$} & \multirow{2}{*}{$\begin{array}{l}\text { Percentage of patients experiencing } \\
\text { recurrence more than } 5 \text { years after } \\
\text { surgery among all patients (no. of } \\
\text { patients) }\end{array}$} \\
\hline & & 3 years & 4 years & 5 years & \\
\hline \multirow[t]{2}{*}{ Liver } & $7.1 \%$ & $89.3 \%$ & $93.8 \%$ & $96.4 \%$ & $0.24 \%$ \\
\hline & $(364)$ & $(301)$ & (316) & $(325)$ & $(12)$ \\
\hline \multirow[t]{2}{*}{ Lung } & $5.5 \%$ & $79.2 \%$ & $89.2 \%$ & $95.8 \%$ & $0.22 \%$ \\
\hline & $(281)$ & $(206)$ & $(232)$ & $(249)$ & $(11)$ \\
\hline \multirow[t]{2}{*}{ Peritoneum } & $2.0 \%$ & $91.3 \%$ & $93.5 \%$ & $95.7 \%$ & $0.09 \%$ \\
\hline & $(100)$ & $(84)$ & $(86)$ & $(88)$ & (4) \\
\hline \multirow[t]{2}{*}{ Local } & $2.0 \%$ & $86.0 \%$ & $95.7 \%$ & $97.9 \%$ & $0.04 \%$ \\
\hline & $(102)$ & $(80)$ & $(89)$ & $(91)$ & (2) \\
\hline \multirow[t]{2}{*}{ Anastomotic } & $1.1 \%$ & $81.1 \%$ & $92.5 \%$ & $98.1 \%$ & $0.02 \%$ \\
\hline & $(55)$ & $(43)$ & $(49)$ & $(52)$ & (1) \\
\hline \multirow[t]{2}{*}{ Other } & $4.8 \%$ & $89.6 \%$ & $93.2 \%$ & $98.2 \%$ & $0.08 \%$ \\
\hline & $(243)$ & (198) & $(206)$ & $(217)$ & (4) \\
\hline All & $18.7 \%$ & & & & \\
\hline$(5103)$ & (953) & & & & \\
\hline
\end{tabular}

JSCCR colorectal cancer registry: (patients in the year 2007); 91 patients were excluded from analyses for cumulative incidence of recurrence because of unknown recurrence date

Table 13 Comparison of the recurrence rates between colon cancer and rectal cancer according to the site of the first recurrence

\begin{tabular}{llll}
\hline Site of recurrence & $\begin{array}{l}\text { Colon cancer } \\
\text { (3135 patients) }\end{array}$ & $\begin{array}{l}\text { Rectal cancer } \\
\text { (1968 patients) }\end{array}$ & $p$ value \\
\hline Liver & $7.2 \%(227)$ & $7.0 \%(137)$ & NS \\
Lung & $3.9 \%(121)$ & $8.1 \%(160)$ & $p<0.0001$ \\
Peritoneum & $2.5 \%(79)$ & $1.1 \%(21)$ & $p=0.0001$ \\
Local & $0.7 \%(22)$ & $4.1 \%(80)$ & $p<0.0001$ \\
Anastomotic & $1.0 \%(30)$ & $1.3 \%(25)$ & NS \\
Other & $4.0 \%(124)$ & $6.1 \%(119)$ & $p=0.0007$ \\
All & $16.0(502)$ & $22.9 \%(451)$ & $p<0.0001$ \\
\hline
\end{tabular}

JSCCR colorectal cancer registry: (patients in the year 2007); RS cancer was counted as rectal cancer

- A past history of colorectal cancer, regardless of stage, is a risk factor for metachronous colorectal cancer [255].

- The recommended interval between colonoscopy ranged from 1 to 5 years, depending on the report [256].

- The need for surveillance targeting multiple primary cancers should be determined by distinguishing hereditary colorectal cancer [257]. There is little evidence of a need for periodic minute examinations for cancer in other organs following surgery for sporadic colorectal cancer (CQ-28).

\section{Clinical questions}

CQ-1: What are the indication criteria for additional treatment after endoscopic resection of pT1 colorectal cancer? (Fig. 11)

(1) Surgical resection is recommended when the vertical margin is positive (Recommendation 1/Evidence level C)

(2) If any of the following findings is observed during histological examination of the resected specimen, intestinal resection with lymph node dissection is recommended as an additional treatment (Recommendation 2/Evidence level B)

(1) $\mathrm{T} 1 \mathrm{~b}$ (depth of SM invasion $\geq 1000 \mu \mathrm{m}$ )

(2) Lymphovascular invasion positive

(3) Poorly differentiated adenocarcinoma, signet-ring cell carcinoma, or mucinous carcinoma [258]

(4) Budding grade of BD2/3 at the site of deepest invasion [258] 
Fig. 11 Treatment strategies for $\mathrm{pT} 1$ cancer after endoscopic resection

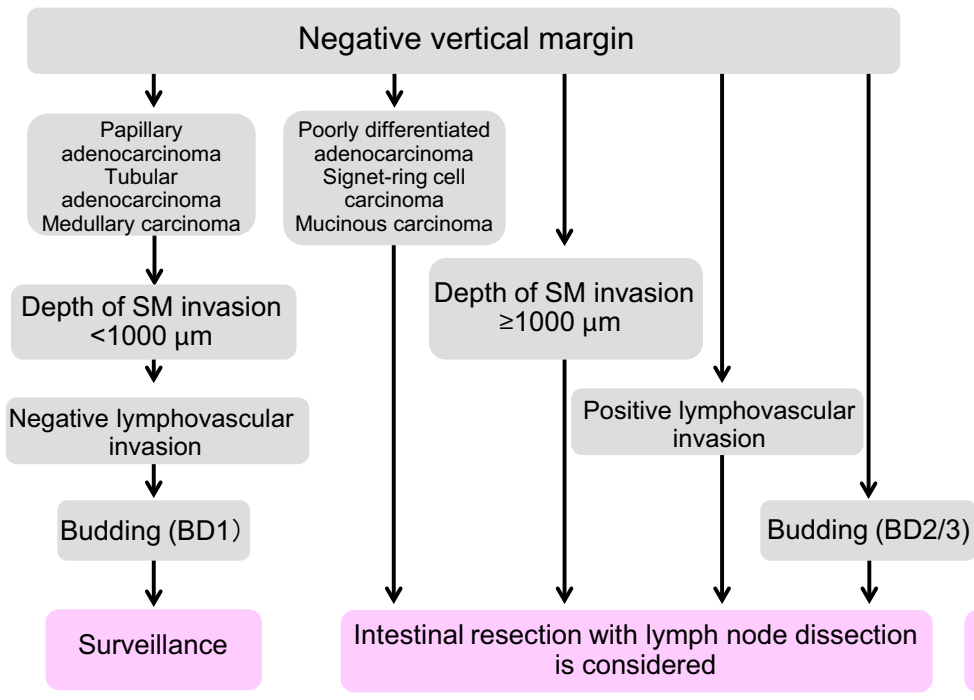

Positive vertical margin
- "Positive vertical margin" means that carcinoma is exposed at the submucosal margin of the resected specimen.

- Depth of SM invasion and the grade of budding are measured by the method described in "Japanese Classification of Colorectal, Appendiceal, and Anal Carcinoma, third English edition" [2].

In cases with a positive vertical margin, the recurrence risk is estimated to be higher if follow-up is carried out in comparison to those with a negative vertical margin, since local remnant cancer is a matter of great concern. Furthermore, it is difficult to make an accurate pathological evaluation of the invasive front of the tumor on resected specimens. Although the evidence level is $\mathrm{C}$, considering the balance between harm and benefit, we decided on a "strong recommendation" based on the result of the vote of the committee.

The principle for treatment of pT1 carcinomas, which are invasive carcinomas, is intestinal resection with lymph node dissection. However, some pT1 (SM) carcinomas have a very low risk of metastasis, and the purpose of these criteria is to minimize the need for additional resections that eventually result in overtreatment of such patients. While no diagnostic methods make it possible to predict lymph node metastasis $(\mathrm{pN})$ without fail, the degree of risk of metastasis can be used as a basis for determining whether or not to perform additional treatment.

Factors such as the depth of submucosal invasion (SM invasion depth) [259], histological type, such as poorly differentiated adenocarcinoma, signet-ring cell carcinoma, and mucinous carcinoma [256], the presence of a poorly differentiated area and muconodules at the site of deepest invasion, budding, and lymphovascular invasion, have been reported to be risk factors for regional lymph node metastasis by pT1 (SM) carcinoma $[258,260]$.
The above criteria for determining whether additional treatment is indicated were prepared based on the following three criteria for performing additional intestinal resection of pT1 (SM) carcinoma described in the "Japanese Classification of Colorectal Carcinoma" (2nd edition, 1980): [(1) Obvious intravascular carcinoma invasion; (2) Poorly differentiated adenocarcinoma or undifferentiated carcinoma; (3) Massive carcinoma invasion extending to the vicinity of the margin] [261]. The description of "Massive carcinoma invasion" in the 4th edition of the "Japanese Classification of Colorectal Carcinoma" was revised to the following more specific description in the 5th edition (1994): "Invasion deeper than 'very shallow invasion' (e.g., invasion exceeding approximately 200-300 $\mu \mathrm{m})$ " [262].

Table 14 Depth of invasion of sm cancer and lymph node metastasis (modified from Ref. [259])

\begin{tabular}{|c|c|c|c|c|}
\hline \multirow{2}{*}{$\begin{array}{l}\text { sm invasion distance } \\
(\mu \mathrm{m})\end{array}$} & \multicolumn{2}{|c|}{ Pedunculated } & \multicolumn{2}{|c|}{ Non-pedunculated } \\
\hline & $\begin{array}{l}\text { Number } \\
\text { of lesions }\end{array}$ & $n(+)(\%)$ & $\begin{array}{l}\text { Number } \\
\text { of lesions }\end{array}$ & $n(+)(\%)$ \\
\hline head invasion & 53 & $3(5.7)$ & & \\
\hline $0<X<500$ & 10 & $0(0)$ & 65 & $0(0)$ \\
\hline $500 \leq X<1000$ & 7 & $0(0)$ & 58 & $0(0)$ \\
\hline $1000 \leq X<1500$ & 11 & $1(9.1)$ & 52 & $6(11.5)$ \\
\hline $1500 \leq X<2000$ & 7 & $1(14.3)$ & 82 & $10(12.2)$ \\
\hline $2000 \leq X<2500$ & 10 & $1(10.0)$ & 84 & $13(15.5)$ \\
\hline $2500 \leq X<3000$ & 4 & $0(0)$ & 71 & $8(11.3)$ \\
\hline $3000 \leq X<3500$ & 9 & $2(22.2)$ & 72 & $5(6.9)$ \\
\hline $3500 \leq X$ & 30 & $2(6.7)$ & 240 & 35 (14.6) \\
\hline
\end{tabular}

The lymph node metastasis rate of patients with a depth of invasion of $1000 \mu \mathrm{m}$ or above was $12.5 \%$

All 3 lymph node metastasis-positive patients with head invasion were lymphatic invasion positive (Ly1) 
Subsequent case series studies in Japan have shown that "200 $\mu \mathrm{m}$ to $300 \mu \mathrm{m}$ " can be extended to $1000 \mu \mathrm{m}$ [263]. According to the results of the project study by the JSCCR, the lymph node metastasis rate of colorectal carcinoma with an SM invasion depth of $1000 \mu \mathrm{m}$ or more was $12.5 \%$ (Table 14) [256, 263]. However, not all cases with submucosal invasion deeper than $1,000 \mu \mathrm{m}$ necessarily require additional surgery. Approximately, $90 \%$ of patients with a depth of invasion of $1000 \mu \mathrm{m}$ or more did not have lymph node metastasis, and it is important to determine whether additional treatment is indicated after sufficiently considering other factors in addition to depth of SM invasion, such as whether other risk factors for lymph node metastasis are present, the physical and social background of the patient, and the patient's wishes.

It has been reported that the incidence of lymph node metastasis is $1.3 \%$ (95\% confidence interval $0-2.4 \%$ ) in cases with an SM invasion degree of $1,000 \mu \mathrm{m}$ or more without risk factors for lymph node metastasis (other than the degree of SM invasion). However, in the event of metastasis or recurrence, a salvage operation cannot be indicated in many cases and cancer death may occur. These risks should be sufficiently discussed among the medical staff, including surgeons.

We added budding as a factor for considering additional treatment in the 2009 edition [264]. Furthermore, project research is currently underway into other histopathological factors. Multicenter joint research projects have produced reports providing the results of consideration into the appropriateness of these criteria [32, 265-267]. Regarding the criteria overseas, the European Society of Gastrointestinal Endoscopy (ESGE) Guideline recommends surgery when lymphovascular invasion, infiltration deeper than $1,000 \mu \mathrm{m}$, positive/nonevaluable vertical margins, or poorly differentiated tumor with submucosal invasion are diagnosed referring to JSGE guidelines [268].

CQ-2: Is endoscopic submucosal dissection (ESD) recommended for lesions with a maximum diameter of $2 \mathrm{~cm}$ or more?

- Endoscopic resection for lesions with a maximum diameter of $2 \mathrm{~cm}$ or more includes EMR, piecemeal EMR, and ESD [33, 269-272].

An accurate preoperative endoscopic diagnosis is essential in endoscopic resection. Selection of EMR, piecemeal EMR, or ESD is determined after taking the operator's skill into consideration.

As a general rule, en bloc resection is recommended for suspected cancer lesions. If en bloc EMR is judged to be difficult, we recommend ESD (en bloc resection) by a skillful endoscopist.
(Recommendation 1/Evidence level B)

CQ-3: Is surveillance recommended after endoscopic resection of early colorectal cancer?

(1) When en bloc endoscopic resection is completed with a negative margin, then it is recommended that surveillance should be performed by endoscopic examination for approximately 1 year for the purpose of searching for any metachronous colon tumors (Recommendation 2/Evidence level B)

(2) When piecemeal endoscopic resection is conducted with a positive horizontal margin, then it is recommended that surveillance should be performed by endoscopic examination for approximately 6 months, as the risks for local recurrence are increased (Recommendation 1/ Evidence level C)

(3) When an additional intestinal resection is not carried out for pT1 cancer, it is recommended that surveillance should be performed via endoscopic examination along with image diagnoses such as CT and tumor markers for the purpose of searching for lymph node metastasis and distant metastasis (Recommendation 1/Evidence level B)

CQ-4: Is laparoscopic surgery recommended for colorectal cancer?

- Laparoscopic surgery is recommended as an option for colorectal cancer surgery (Recommendation 2/Evidence level B).

However, the patient should be instructed that the efficacy of laparoscopic surgery for transverse colon cancer and rectal cancer is not well established.

The difficulty for locally advanced cancer and patients with obesity and adhesion is high, so the indications should be determined while taking into consideration the skill of each surgical team.

CQ-5: Is lateral lymph node dissection recommended for rectal cancer?

Lateral lymph node dissection is indicated when the lower border of the tumor is located distal to the peritoneal reflection and the tumor has invaded beyond the muscularis propria. The diagnostic criteria for lateral lymph node metastasis have not been established. At present, the criteria for cases where lateral lymph node dissection can be omitted are not clear.

(1) It is recommended that lateral lymph node dissection should be performed if a preoperative or intraoperative 
diagnosis reveals the presence of lateral lymph node metastasis (Recommendation 1/Evidence level C)

(2) Lateral lymph node dissection is recommended, even if lateral lymph node metastasis is not detected by a preoperative or intraoperative diagnosis. Although the survival benefit of lateral lymph node dissection in this group of patients is limited, it can be expected to suppress local recurrence (Recommendation 2/Evidence level B)

\section{Comments}

According to retrospective studies in Japan, lateral lymph node metastasis exists in 16-23\% of cases of lower rectal cancer (Table 6) [40, 273-276]. Although the prognosis of these cases is poor, in general, $40-50 \%$ of patients with R0 resection reportedly achieved five-year survival $[40,274$, 276-280]. The efficacy of lateral lymph node dissection is particularly high for patients with lateral nodal involvement in whom the number of lymph node metastases or the number of involved lateral lymph node station is limited [281, 282]. A propensity score matching analysis of pT3/T4 lower rectal cancer cases in the 1995-2004 JSCCR colorectal cancer registry also showed that the 5-year overall survival rate of patients with lateral lymph node dissection was better than that of those without dissection (68.9\% vs. 62.0\%) [283]. It is considered that there is a high likelihood of achieving a survival improvement by lateral lymph node dissection. Although the evidence level is $\mathrm{C}$, considering the balance between harm and benefit, this was made a "strong recommendation" based on the result of a vote by the committee.

It has been reported that the incidence of lateral lymph node metastasis remains high after preoperative chemoradiation therapy if the lateral lymph nodes are enlarged before treatment. Thus, even in cases in which preoperative chemoradiotherapy is performed, the omission of lateral lymph node dissection is not recommended [284, 285].

Regarding the clinical value of lateral lymph node dissection in cases without obvious lateral lymph node metastasis, the JCOG0212 study examined the non-inferiority of the mesorectal excision (ME) alone to the mesorectal excision with lateral lymph node dissection (ME + LLND) with the primary endpoints of relapse-free survival. This study was conducted for patients with no lateral lymph nodes with a short-axis diameter of $10 \mathrm{~mm}$ or more on preoperative CT or MRI and whose tumor was located in the rectum, with the lower tumor margin below the peritoneal reflection. As a result, the non-inferiority of ME alone to ME + LLND was not statistically proven $(\mathrm{P}$ value for non-inferiority $=0.0547$ ) [286]. The frequency of local recurrence in the ME + LLND group was significantly lower than that in the ME alone group $(7.4 \%$ vs. $12.6 \%)$. On the other hand, the relapsefree survival curves of the two groups were very similar, and there was no significant difference in either the overall survival rate or local recurrence-free survival rate as a secondary endpoint. Thus, the survival benefit of lateral lymph node dissection was limited in cases without lateral lymph node enlargement. Taken together, the omission of lateral lymph node dissection is not uniformly recommended, even for cases without the enlargement of lateral pelvic lymph nodes, from the viewpoint of local control. The application of lateral lymph node dissection should be determined in individual patients by comprehensively considering the balance between the expected benefits in terms of local control and survival improvement and the surgical risk and postoperative dysfunction.

CQ-6: Is resection of the primary tumor recommended for patients with unresectable distant metastases?

- If symptoms exist as a result of the primary tumor, which are difficult to control using other therapies, and the resection is not significantly invasive, primary tumor resection and early systemic therapy are recommended (Recommendation 1/Evidence level C)

For cases in which no symptoms are caused by the primary tumor, however, the efficacy of resecting the primary tumor has not been established.

CQ-7: In cases where peritoneal metastasis is noted, is the resection of peritoneal metastasis at the same time as the primary lesion recommended?

- If the metastasis is localized $(\mathrm{P} 1, \mathrm{P} 2)$ and the resection is not significantly invasive, then the peritoneal metastasis should be resected at the same time as the primary tumor (Recommendation 1/Evidence level C)

\section{Comments}

Some cases of long-term survival have been reported in which localized peritoneal metastasis $(\mathrm{P} 1, \mathrm{P} 2)$ was resected alongside the primary tumor [287-290].

Simultaneous localized dissemination (P1, P2) that can be excised without excessive risk should be resected along with the primary tumor. It should be noted that it is more effective to excise localized dissemination $(\mathrm{P} 1, \mathrm{P} 2)$ without hematogenous metastasis together with the primary tumor [291, 292]. It is considered that improved survival can be highly expected. Thus, it was decided that this should be a "strong recommendation," although the evidence level is C.

CQ-8: Is resection recommended for cases in which metastases are simultaneously noted in the liver and lungs? 
- The efficacy of resection in patients who have liver and lung metastases at the same time has been shown and, thus, resection should be considered for patients with resectable liver and lung metastases (Recommendation 2/Evidence level D)

CQ-9: Is neoadjuvant chemotherapy recommended for cases with resectable liver metastasis?

- The efficacy and safety of neoadjuvant chemotherapy for resectable liver metastasis have not been established (No recommendation/Evidence level C)

CQ-10: Is resection of liver/lung metastasis recommended, if it becomes possible as a result of the effects of chemotherapy?

- Resection should be performed for cases in which chemotherapy has successfully made localized metastasis to the liver or lungs operable (Recommendation 2/Evidence level C)

CQ-11: Is resection of liver metastasis recommended, if it becomes invisible as a result of the effects of chemotherapy?

- Resection is recommended if liver metastasis is no longer visible on both CT and MRI after chemotherapy (Recommendation 2/Evidence level D)

\section{Comments}

Approximately, 20-25\% of metastatic liver lesions have been reported to disappear after 6-12 courses of medication. However, even if a complete response is observed on imaging, a pathological complete response (disappearance of tumor cells) is not always obtained [293]. There is a high possibility that tumor cells will remain and it is, therefore, recommended that site of the disappearing liver metastasis be excised [294-297].

CQ-12: Is laparoscopic surgery recommended for liver metastasis of colorectal cancer?

- If a well-experienced surgical team carefully considers adaptation, the safety of laparoscopic hepatectomy for colorectal cancer liver metastasis has been confirmed to be nearly equivalent to that of laparotomy. However, with respect to efficacy, the evidence is insufficient and it is not a standard surgical procedure for liver metasta- sis of colorectal cancer (No recommendation/Evidence level D)

CQ-13: Is thermal ablation therapy recommended for metastatic liver lesions?

There are few reports indicating the efficacy of thermal ablation therapy. Since thermal ablation therapy is accompanied by a high risk of local recurrence in cases of liver metastasis, resection should be initially considered wherever possible.

(1) As resection is the standard therapy for resectable lesions, it is not recommended as the first choice of treatment (Recommendation 1/Evidence level C)

(2) As systemic therapy is the standard therapy for unresectable liver metastasis, it is not recommended for unresectable lesions (Recommendation 2/Evidence level C)

CQ-14: Is surgical resection recommended in cases with locally recurrent rectal cancer?

- Resection is recommended for local recurrence of rectal cancer when R0 resection is considered possible (Recommendation 2/Evidence level C)

The indication of resection should be decided after considering the surgical stress, risk, and postoperative quality of life.

It is necessary to fully consider the proficiency of the individual surgical team if pelvic exenteration and bony pelvic wall resection are expected.

CQ-15: is postoperative adjuvant chemotherapy recommended for Stage III colorectal cancer?

(1) Oxaliplatin combination therapy is recommended for Stage III colon cancer (Recommendation 1/Evidence level A)

(2) Fluoropyrimidine monotherapy is recommended for Stage III colon cancer (Recommendation 2/Evidence level A)

Treatment selection according to recurrence Recurrence risk Low risk High risk

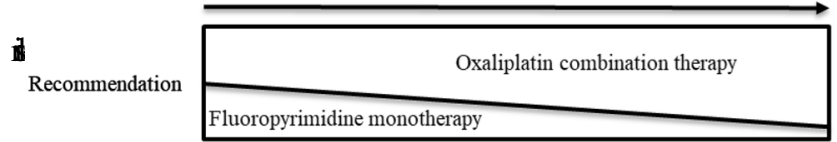

Comments

In postoperative adjuvant chemotherapy for Stage III colon cancer, oxaliplatin (OX) combination therapy reduces the relative risk of relapse/death by approximately $20 \%$ in 
comparison to $5-\mathrm{FU}+l-\mathrm{LV}$, which has been reproducibly confirmed by three RCTs in Europe and the United States $[142,143,298-300]$. Thus, it is recommended as the most effective treatment option.

On the other hand, in an integrated analysis of three randomized controlled trials in Europe and the United States targeting Dukes' B and Dukes' C, 5 -FU $+l$-LV was associated with significantly better relapse-free survival and overall survival in comparison to surgery alone [301]. Subsequently, in domestic and international randomized controlled trials, the non-inferiority of Cape (X-ACT [302]) and UFT + LV (NSABP C-06 [303], JCOG 0205 [144]) to $5-\mathrm{FU}+l$-LV was shown, followed by the noninferiority of S-1 to UFT + LV (ACTS-CC [145]). From these facts, it is considered that each of the above-mentioned fluoropyrimidine monotherapies (i.e., $5-\mathrm{FU}+l-\mathrm{LV}$, Cape, UFT + LV, S-1) has a survival benefit in comparison to surgery alone. However, its effect has been shown to be inferior to OX combination therapy, as described above.

Upon selecting the actual treatment regimen, the risk of recurrence and the expected effect in each patient should be considered (see above figure). In addition, adequate information, such as adverse events, treatment costs, and hospital visits, should be provided to each patient. It is desirable to select therapy based on comprehensive judgment, including the patient's general condition and willingness to treat.

CQ-16: Is the recommended duration of postoperative adjuvant chemotherapy 6 months?

(1) It is recommended that postoperative adjuvant chemotherapy should be performed for 6 months (Recommendation 1/Evidence level A)

(2) However, if CAPOX therapy is used for low-risk colon cancer, it is recommended that postoperative adjuvant chemotherapy should be performed for 3 months (Recommendation 2/Evidence level A)

CQ-17: Is postoperative adjuvant chemotherapy recommended in patients aged 70 or over?

- Even in patients 70 years old or older, postoperative adjuvant chemotherapy is recommended if their PS is good, if the function of major organs is adequate, and if there are no complications that may be a risk for performing chemotherapy (Recommendation 1/Evidence level A)

CQ-18: Is postoperative adjuvant chemotherapy recommended for Stage II colorectal cancer?
(1) Postoperative adjuvant chemotherapy is recommended for high-risk Stage II patients (Recommendation 2/Evidence level B)

(2) However, postoperative adjuvant chemotherapy is not recommended for other Stage II patients (Recommendation 2/Evidence level B)

CQ-19: Is adjuvant chemotherapy recommended subsequent to the resection of a distant metastatic lesion?

(1) Adjuvant chemotherapy is recommended for patients after the curative resection of liver metastases (Recommendation 2/Evidence level B)

(2) Adjuvant chemotherapy is recommended for patients after curative resection of distant metastases other than liver metastasis (e.g., lung metastases) (Recommendation 2/Evidence level D)

CQ-20: Is concomitant therapy with molecular targeted drugs recommended as a first-line therapy?

- Usage in combination with either Bevacizumab or antiEGFR antibody drug is recommended (Recommendation 1/Evidence level A)

\section{Comments}

The efficacy and safety of combination therapy with molecular targeted drugs as first-line therapy for unresectable colorectal cancer have been demonstrated for bevacizumab (BEV), cetuximab (CET) and panitumumab (PANI). On the other hand, ramucirumab (RAM), aflibercept beta (AFL) and regorafenib (REG) have not been confirmed in firstline therapy, and their concomitant use is not recommended [193, 194, 214].

Recently, in the pooled analysis of six RCTs (FIRE-3 trial, CALGB/SWOG 80405, PEAK, CRYSTAL, PRIME, 20050181) for $R A S$ wild-type unresectable colorectal cancer, a correlation between the tumor location (right side or left side) and the therapeutic effect of molecular targeted drugs (BEV or anti-EGFR antibody) was reported [220]. Based on the results of the analysis, anti-EGFR antibody therapies are recommended for $R A S / B R A F$ wild-type colon cancer when the primary lesion is on the left side, while $\mathrm{BEV}$ is recommended for cases in which the primary lesion is located on the right side [218]. On the other hand, $\mathrm{BEV}$ combination therapy is recommended for $R A S$ or $B R A F$ mutated colon cancer, regardless of the location of the primary lesion [218]. In $B R A F$-mutated colorectal cancer, FOLFOXIRI + BEV combination therapy has shown high efficacy. Thus, FOLFOXIRI + BEV is recommended 
as the first choice if it can be applied, considering the age, PS and comorbidities of the patient [175].

Taken together, chemotherapy in combination with BEV or anti-EGFR antibody drugs is recommended as first-line therapy for unresectable colorectal cancer, unless contraindicated. For $R A S / B R A F$ wild-type, either BEV or anti-EGR antibody drugs should be selected considering the toxicity profile, backbone chemotherapies, patient preference, and primary tumor location. Since the efficacy of anti-EGFR antibody drugs varies according to the RAS/ $B R A F$ genotype, it is desirable to perform mutation testing of $R A S$ and $B R A F$ prior to the selection of the first-line therapy.

CQ-21: Is concomitant therapy with molecular targeted drugs recommended as a second-line therapy?

(1) Usage in combination with an anti-VEGF antibody drug is recommended (Recommendation 1/Evidence level A)

(2) Usage in combination with an anti-EGFR antibody drug is recommended (Recommendation 2/Evidence level A)

CQ-22: For third or later line treatments, is the administration of Regorafenib or FTD/TPI recommended?

- If a patient becomes non-responsive or intolerant to fluoropyrimidine, oxaliplatin, irinotecan, either drug is recommended as salvage line therapy.

\section{(Recommendation level 1/Evidence level A)}

CQ-23: Are immune checkpoint inhibitors recommended for colorectal cancer?

- Anti-PD-1 antibody therapy is recommended for MSI-H unresectable colorectal cancer patient who have undergone previous treatment (Recommendation level 1/Evidence level B)

CQ-24: Is hepatic arterial infusion therapy recommended in cases of liver metastasis?

- When systemic therapy is available, it is recommended that hepatic arterial infusion therapy not be performed for the treatment of unresectable liver metastasis (Recommendation 1/Evidence level C)

CQ-25: Is neoadjuvant therapy recommended for patients with $\mathrm{R} 0$ resectable rectal cancer?
(1) For rectal cancer with a high risk of local recurrence, preoperative chemoradiotherapy is recommended (Recommendation 2/Evidence level B)

(2) The efficacy of preoperative chemotherapy (without radiation) has not been established. It is recommended that preoperative chemotherapy not be performed (Recommendation 2/Evidence level C)

CQ-26: Is chemoradiotherapy recommended for unresectable locally advanced and locally recurrent rectal cancer without distant metastasis?

- Chemoradiotherapy directed to resection is recommended for cases in which R0 resection is expected to be possible due to tumor shrinkage (Recommendation 2/Evidence level B)

On the other hand, it is considered reasonable to carry out systemic therapy for the purpose of continuous tumor control when resection cannot be expected. Regarding irradiation of local lesions, it is desirable to consider the symptoms, expected effects, and predicted adverse events.

CQ-27: Is stent treatment recommended for obstructive colorectal cancer?

(1) Stent treatment for symptomatic relief in patients who are not indicated for systemic therapy is recommended as a treatment option with a reduced physical and psychological burden on patients (Recommendation 2/Evidence level B)

(2) Stent treatment is not recommended for patients who are indicated for systemic therapy (Recommendation 2/ Evidence level B)

(3) Obstruction relief by stent treatment as a bridge to surgery (BTS) premised on curative surgical removal avoids emergency surgery and reduces the risk of postoperative complications. However, it is also pointed out that perforation and other adverse effects may worsen the long-term prognosis (No recommendation/Evidence level C)

CQ-28: Is the surveillance of multiple primary cancers (multiple colorectal cancer or other organ cancer) recommended after curative surgery for colorectal cancer?

(1) Metachronous colorectal cancer occurs more frequently in cases of colorectal cancer resection than in the general population and, as such, regular colonoscopic examination of the large bowel is recommended (Recommendation 1/Evidence level B) 
(2) There is no indication that post-surgical surveillance targeting multiple primary cancers in other organs is effective. As such, it is recommended that no examination be conducted for this purpose. The appropriate course of action is to educate the patient regarding the need for regular cancer examinations and to recommend that they undergo such examinations (Recommendation 2/ Evidence level C)

For hereditary colorectal cancer, it is necessary to carry out surveillance for multiple primary cancers under appropriate counseling (see "JSCCR Guidelines 2016 for the Clinical Practice of Hereditary Colorectal Cancer") [227].

Funding Preparation of these Guidelines was funded by the JSCCR. No financial support was received from any other organization or corporation.

\section{Compliance with ethical standards}

Conflict of interest (1) The following corporations were disclosed by self-declaration of the Guideline Committee members and Guideline Evaluation Committee members. (2) A2 Healthcare Corp., Akita Sumitomo Co., Ltd., Array Biopharma Inc., Astellas Pharma Inc., Bayer Yakuhin, Ltd., Bristol-Myers Squibb Company, Chugai Pharmaceutical Co., Ltd., Covidien Japan, Inc., DAIICHI SANKYO COMPANY, Ltd., EA Pharma Co., Ltd., Eisai Co., Ltd., Eli Lilly Japan K.K., Gilead Sciences, Inc., Intuitive Surgical, Inc., Japan Clinical Research Operations, Johnson \& Johnson K.K., Kaigen Pharma Co., Ltd., Kyowa Hakko Kirin Co., Ltd., Mediscience Planning Inc., Merck Serono Co., Ltd., Merck Biopharma Co., Ltd., Mitsubishi Tanabe Pharma Corporation, MRP Co., Ltd., MSD K.K., NanoCarrier Co., Ltd., Nippon Boehringer lngelheim Co., Ltd., Olympus Corporation, Ono pharmaceutical Co., Ltd., Otsuka Pharmaceutical Co., Ltd., Pfizer Japan Inc., Sanofi K.K., Shimadzu Corporation, Shionogi \& Co., Ltd., Sumitomo Dainippon Pharma Co., Ltd., Sysmex Corporation, Taiho pharmaceutical Co., Ltd., Takeda Pharmaceutical Company Limited., TERUMO CORPORATION, Yakult Honsha Co., Ltd. ((2) Measures for the conflicts of interest:The Guideline Committee and the Guideline Evaluation Committee have been organized in members with a diverse range of disciplines, including surgery, internal medicine, radiology, pathology, etc., in order to minimize biased opinion. Each recommendation was made determined not on an individual opinion basis but based on voting by the whole committee members, with consensus prioritized. When voting for $\mathrm{CQ}$, conflicts in relation to economic and academic interests were confirmed for each CQ, and members with conflicts of interest in the CQ abstained from voting.

Open Access This article is distributed under the terms of the Creative Commons Attribution 4.0 International License (http://creativeco mmons.org/licenses/by/4.0/), which permits unrestricted use, distribution, and reproduction in any medium, provided you give appropriate credit to the original author(s) and the source, provide a link to the Creative Commons license, and indicate if changes were made.

\section{References}

1. Japanese Society for Cancer of the Colon and Rectum (2016) JSCCR guidelines 2016 for the treatment of colorectal cancer. Kanehara \& Co., Ltd., Tokyo

2. Japanese Society for Cancer of the Colon and Rectum (2019) Japanese classification of colorectal, appendiceal, and anal carcinoma, third, English edn. Kanehara \& CO., Ltd., Tokyo

3. Fukui T, Yamaguchi N, Morizane T (2014) Minds handbook for clinical practice guideline development 2014. Igaku Shoin, Tokyo

4. Aihara M, Mihara H, Murayama T (2010) GRADE system for clinical practice guideline-therapeutic intervention. Toppan Media, Hirosaki

5. Atkins D, Eccles M, Flottorp S et al (2004) Systems for grading the quality of evidence and the strength of recommendations I: critical appraisal of existing approaches The GRADE Working Group. BMC Health Serv Res 4:38

6. Guyatt GH, Oxman AD, Vist GE et al (2008) GRADE: an emerging consensus on rating quality of evidence and strength of recommendations. BMJ 336:924-926

7. Guyatt GH, Oxman AD, Kunz R et al (2008) What is "quality of evidence" and why is it important to clinicians? BMJ 336:995998

8. Schunemann HJ, Oxman AD, Brozek J et al (2008) Grading quality of evidence and strength of recommendations for diagnostic tests and strategies. BMJ 336:1106-1110

9. Guyatt GH, Oxman AD, Kunz R et al (2008) Incorporating considerations of resources use into grading recommendations. BMJ 336:1170-1173

10. Guyatt GH, Oxman AD, Kunz R et al (2008) Going from evidence to recommendations. BMJ 336:1049-1051

11. Jaeschke R, Guyatt GH, Dellinger P et al (2008) Use of GRADE grid to reach decisions on clinical practice guide- lines when consensus is elusive. BMJ 337:a744

12. Guyatt G, Oxman AD, Akl EA et al (2011) GRADE guidelines: 1. Introduction-GRADE evidence profiles and summary of findings tables. J Clin Epidemiol 64:383-394

13. Guyatt GH, Oxman AD, Kunz R et al (2011) GRADE guidelines: 2. Framing the question and deciding on important out-comes. J Clin Epidemiol 64:395-400

14. Balshem H, Helfand M, Schunemann HJ et al (2011) GRADE guidelines: 3. Rating the quality of evidence. J Clin Epidemiol 64:401-406

15. Guyatt GH, Oxman AD, Vist $G$ et al (2011) GRADE guidelines: 4. Rating the quality of evidence-study limitations (risk of bias). J Clin Epidemiol 64:407-415

16. Guyatt GH, Oxman AD, Montori V et al (2011) GRADE guidelines: 5 . Rating the quality of evidence-publication bias. J Clin Epidemiol 64:1277-1282

17. Guyatt GH, Oxman AD, Kunz R et al (2011) GRADE guidelines 6. Rating the quality of evidence-imprecision. J Clin Epidemiol 64:1283-1293

18. Guyatt GH, Oxman AD, Kunz R et al (2011) GRADE guidelines: 7. Rating the quality of evidence-inconsistency. J Clin Epidemiol 64:1294-1302

19. Guyatt GH, Oxman AD, Kunz R et al (2011) GRADE guidelines: 8. Rating the quality of evidence-indirectness. J Clin Epidemiol 64:1303-1310

20. Guyatt GH, Oxman AD, Sultan S et al (2011) GRADE guidelines: 9. Rating up the quality of evidence. J Clin Epidemiol 64:1311-1316

21. Brunetti M, Shemilt I, Pregno S et al (2013) GRADE guidelines: 10. Considering resource use and rating the quality of economic evidence. J Clin Epidemiol 66:140-150 
22. Guyatt G, Oxman AD, Sultan S et al (2013) GRADE guidelines: 11. Making an overall rating of confidence in effect estimates for a single outcome and for all outcomes. J Clin Epidemiol 66:151-157

23. Guyatt GH, Oxman AD, Santesso N et al (2013) GRADE guidelines: 12. Preparing summary of findings tables-binary outcomes. J Clin Epidemiol 66:158-172

24. Guyatt GH, Thorlund K, Oxman AD et al (2013) GRADE guidelines: 13. Preparing summary of findings tables and evidence profiles-continuous outcomes. J Clin Epidemiol 66:173-183

25. Andrews J, Guyatt G, Oxman AD et al (2013) GRADE guidelines: 14. Going from evidence to recommendations: the significance and presentation of recommendations. J Clin Epidemiol 66:719-725

26. Andrews JC, Schunemann HJ, Oxman AD et al (2013) GRADE guidelines: 15 . Going from evidence to recommendation-determinants of a recommendation's direction and strength. J Clin Epidemiol 66:726-735

27. Iwatate M, Sano Y, Tanaka S et al (2018) Validation study for development of the Japan NBI Expert Team classification of colorectal lesions. Dig Endosc 30:642-651

28. Saitoh Y, Obara T, Watari J et al (1998) Invasion depth diagnosis of depressed type early colorectal cancers by combined use of videoendoscopy and chromoendoscopy. Gastrointest Endosc 48:362-370

29. Watari J, Saitoh Y, Obara T et al (1997) Early nonpolypoid colorectal cancer: radiographic diagnosis of depth of invasion. Radiology 205:67-74

30. Tanaka S, Kaltenbach T, Chayama K et al (2006) High-magnification colonoscopy (with videos). Gastrointest Endosc 64:604-613

31. Tanaka S, Oka S, Chayama K (2008) Colorectal endoscopic submucosal dissection: present status and future perspective, including its differentiation from endoscopic mucosal resection. J Gastroenterol 43:641-651

32. Oka S, Tanaka S, Kanao H et al (2011) Mid-term prognosis after endoscopic resection for submucosal colorectal carcinoma: summary of a multicenter questionnaire survey conducted by the colorectal endoscopic resection standardization implementation working group in Japanese Society for Cancer of the Colon and Rectum. Dig Endosc 23:190-194

33. Tanaka S, Kashida H, Saito Y et al (2015) JGES guidelines for colorectal endoscopic submucosal dissection/endoscopic mucosal resection. Dig Endosc 27:417-434

34. Kotake K, Mizuguchi T, Moritani K et al (2014) Impact of D3 lymph node dissection on survival for patients with T3 and T4 colon cancer. Int J Colorectal Dis 29:847-852

35. Japanese Society for Cancer of the Colon and Rectum: Multiinstitutional registry of large bowel cancer in Japan (2012) Cases treated in 2000-2002, vol 29 (2011), cases treated in 2003-2004, vol $30(2012)$

36. Heald RJ, Husband EM, Ryall RD (1982) The mesorectum in rectal cancer surgery-the clue to pelvic recurrence? Br J Surg 69:613-616

37. MacFarlane JK, Ryall RD, Heald RJ (1993) Mesorectal excision for rectal cancer. Lancet 341:457-460

38. Enker WE, Thaler HT, Cranor ML et al (1995) Total mesorectal excision in the operative treatment of carcinoma of the rectum. J Am Coll Surg 181:335-346

39. Lowry AC, Simmang CL, Boulos P et al (2001) Consensus statement of definitions for anorectal physiology and rectal cancer: report of the Tripartite Consensus Conference on Definitions for Anorectal Physiology and Rectal Cancer, Washington, D.C., May 1, 1999. Dis Colon Rectum 44:915-919

40. Sugihara K, Kobayashi H, Kato T et al (2006) Indication and benefit of pelvic sidewall dissection for rectal cancer. Dis Colon Rectum 49:1663-1672
41. Hida J, Yasutomi M, Maruyama T et al (1997) The extent of lymph node dissection for colon carcinoma: the potential impact on laparoscopic surgery. Cancer 80:188-192

42. Ono C, Yoshinaga K, Enomoto M et al (2002) Discontinuous rectal cancer spread in the mesorectum and the optimal distal clearance margin in situ. Dis Colon Rectum 45:744-749 (discussion 742-743)

43. Shimada Y, Takii Y, Maruyama S et al (2011) Intramural and mesorectal distal spread detected by whole-mount sections in the determination of optimal distal resection margin in patients undergoing surgery for rectosigmoid or rectal cancer without preoperative therapy. Dis Colon Rectum 54:1510-1520

44. Shirouzu K, Isomoto H, Kakegawa T (1995) Distal spread of rectal cancer and optimal distal margin of resection for sphincterpreserving surgery. Cancer 76:388-392

45. Zhao GP, Zhou ZG, Lei WZ et al (2005) Pathological study of distal mesorectal cancer spread to determine a proper distal resection margin. World J Gastroenterol 11:319-322

46. Martin ST, Heneghan HM, Winter DC (2012) Systematic review of outcomes after intersphincteric resection for low rectal cancer. Br J Surg 99:603-612

47. Pollack J, Holm T, Cedermark B et al (2006) Long-term effect of preoperative radiation therapy on anorectal function. Dis Colon Rectum 49:345-352

48. Lange MM, den Dulk M, Bossema ER et al (2007) Risk factors for faecal incontinence after rectal cancer treatment. Br J Surg 94:1278-1284

49. Yamada K, Ogata S, Saiki Y et al (2009) Long-term results of intersphincteric resection for low rectal cancer. Dis Colon Rectum 52:1065-1071

50. Fujita S, Akasu T, Mizusawa J et al (2012) Postoperative morbidity and mortality after mesorectal excision with and without lateral lymph node dissection for clinical stage II or stage III lower rectal cancer (JCOG0212): results from a multicentre, randomised controlled, non-inferiority trial. Lancet Oncol 13:616-621

51. Saito S, Fujita S, Mizusawa J et al (2016) Male sexual dysfunction after rectal cancer surgery: results of a randomized trial comparing mesorectal excision with and without lateral lymph node dissection for patients with lower rectal cancer: Japan Clinical Oncology Group Study JCOG0212. Eur J Surg Oncol 42:1851-1858

52. Ito M, Kobayashi A, Fujita S et al (2018) Urinary dysfunction after rectal cancer surgery: results from a randomized trial comparing mesorectal excision with and without lateral lymph node dissection for clinical stage II or III lower rectal cancer (Japan Clinical Oncology Group Study, JCOG0212). Eur J Surg Oncol 44:463-468

53. de Santibanes E, Lassalle FB, McCormack L et al (2002) Simultaneous colorectal and hepatic resections for colorectal cancer: postoperative and longterm outcomes. J Am Coll Surg 195:196-202

54. Nakai N, Yamaguchi T, Kinugasa Y et al (2017) Long-term outcomes after resection of para-aortic lymph node metastasis from left-sided colon and rectal cancer. Int J Colorectal Dis 32:999-1007

55. Min BS, Kim JS, Kim NK et al (2009) Extended lymph node dissection for rectal cancer with radiologically diagnosed extramesenteric lymph node metastasis. Ann Surg Oncol 16:3271-3278

56. Choi PW, Kim HC, Kim AY et al (2010) Extensive lymphadenectomy in colorectal cancer with isolated para-aortic lymph node metastasis below the level of renal vessels. J Surg Oncol 101:66-71

57. Arimoto A, Uehara K, Kato T et al (2015) Clinical significance of para-aortic lymph node dissection for advanced or metastatic 
colorectal cancer in the current era of modern chemotherapy. Dig Surg 32:439-444

58. Bae SU, Han YD, Cho MS et al (2016) Oncologic outcomes of colon cancer patients with extraregional lymph node metastasis: comparison of isolated paraaortic lymph node metastasis with resectable liver metastasis. Ann Surg Oncol 23:1562-1568

59. Murata S, Moriya Y, Akasu T et al (1998) Resection of both hepatic and pulmonary metastases in patients with colorectal carcinoma. Cancer 83:1086-1093

60. Kobayashi K, Kawamura M, Ishihara T (1999) Surgical treatment for both pulmonary and hepatic metastases from colorectal cancer. J Thorac Cardiovasc Surg 118:1090-1096

61. Robinson BJ, Rice TW, Strong SA et al (1999) Is resection of pulmonary and hepatic metastases warranted in patients with colorectal cancer? J Thorac Cardiovasc Surg 117:66-75 (discussion 75-76)

62. Lambert LA, Colacchio TA, Barth RJ Jr (2000) Interval hepatic resection of colorectal metastases improves patient selection. Arch Surg 135:473-479 (discussion 479-480)

63. Yoshidome H, Kimura F, Shimizu $\mathrm{H}$ et al (2008) Interval period tumor progression: does delayed hepatectomy detect occult metastases in synchronous colorectal liver metastases? J Gastrointest Surg 12:1391-1398

64. Adam R (2007) Developing strategies for liver metastases from colorectal cancer. Semin Oncol 34:S7-S11

65. Lam VW, Spiro C, Laurence JM et al (2012) A systematic review of clinical response and survival outcomes of down-sizing systemic chemotherapy and rescue liver surgery in patients with initially unresectable colorectal liver metastases. Ann Surg Oncol 19:1292-1301

66. Min BS, Kim NK, Sohn SK et al (2008) Isolated paraaortic lymph-node recurrence after the curative resection of colorectal carcinoma. J Surg Oncol 97:136-140

67. Wong JS, Tan GH, Teo MC (2016) Management of para-aortic lymph node metastasis in colorectal patients: a systemic review. Surg Oncol 25:411-418

68. Nagata H, Ishihara S, Hata K et al (2017) Survival and prognostic factors for metachronous peritoneal metastasis in patients with colon cancer. Ann Surg Oncol 24:1269-1280

69. Franzese C, Fogliata A, Comito T et al (2017) Stereotactic/hypofractionated body radiation therapy as an effective treatment for lymph node metastases from colorectal cancer: an institutional retrospective analysis. Br J Radiol 90:20170422

70. Isozaki Y, Yamada S, Kawashiro S et al (2017) Carbon-ion radiotherapy for isolated para-aortic lymph node recurrence from colorectal cancer. J Surg Oncol 116:932-938

71. Kim MS, Cho CK, Yang KM et al (2009) Stereotactic body radiotherapy for isolated paraaortic lymph node recurrence from colorectal cancer. World J Gastroenterol 15:6091-6095

72. Tschmelitsch J, Kronberger P, Glaser K et al (1994) Survival after surgical treatment of recurrent carcinoma of the rectum. J Am Coll Surg 179:54-58

73. Tanis PJ, Doeksen A, van Lanschot JJ (2013) Intentionally curative treatment of locally recurrent rectal cancer: a systematic review. Can J Surg 56:135-144

74. Martin LW, Warren RS (2000) Current management of colorectal liver metastases. Surg Oncol Clin N Am 9:853-876 (discussion 877-858)

75. Penna C, Nordlinger B (2002) Colorectal metastasis (liver and lung). Surg Clin North Am 82(1075-1090):x-xi

76. Abdalla EK, Vauthey JN, Ellis LM et al (2004) Recurrence and outcomes following hepatic resection, radiofrequency ablation, and combined resection/ablation for colorectal liver metastases. Ann Surg 239:818-825 (discussion 825-817)

77. Nordlinger B, Sorbye H, Glimelius B et al (2013) Perioperative FOLFOX4 chemotherapy and surgery versus surgery alone for resectable liver metastases from colorectal cancer (EORTC 40983): long-term results of a randomised, controlled, phase 3 trial. Lancet Oncol 14:1208-1215

78. Kato T, Yasui K, Hirai T et al (2003) Therapeutic results for hepatic metastasis of colorectal cancer with special reference to effectiveness of hepatectomy: analysis of prognostic factors for 763 cases recorded at 18 institutions. Dis Colon Rectum 46:S22-S31

79. Niekel MC, Bipat S, Stoker J (2010) Diagnostic imaging of colorectal liver metastases with CT, MR imaging, FDG PET, and/or FDG PET/CT: a meta-analysis of prospective studies including patients who have not previously undergone treatment. Radiology 257:674-684

80. Moulton CA, Gu CS, Law CH et al (2014) Effect of PET before liver resection on surgical management for colorectal adenocarcinoma metastases: a randomized clinical trial. JAMA 311:1863-1869

81. Fortner JG, Silva JS, Golbey RB et al (1984) Multivariate analysis of a personal series of 247 consecutive patients with liver metastases from colorectal cancer. I. Treatment by hepatic resection. Ann Surg 199:306-316

82. Hughes KS, Simon R, Songhorabodi S et al (1986) Resection of the liver for colorectal carcinoma metastases: a multi-institutional study of patterns of recurrence. Surgery 100:278-284

83. Ekberg H, Tranberg KG, Andersson R et al (1987) Pattern of recurrence in liver resection for colorectal secondaries. World $\mathrm{J}$ Surg 11:541-547

84. Battula N, Tsapralis D, Mayer D et al (2014) Repeat liver resection for recurrent colorectal metastases: a single-centre, 13-year experience. HPB (Oxford) 16:157-163

85. Nordinger B, Jaeck D, Guiguet M et al (1992) Surgical resection of hepatic metastases. Multicentric retrospective study by the French Association of Surgery In: Nordinger B, Jaeck D (eds) Treatment of hepatic metastases of colorectal cancer. Springer, Paris, pp 129-46

86. Cady B, Jenkins RL, Steele GD Jr et al (1998) Surgical margin in hepatic resection for colorectal metastasis: a critical and improvable determinant of outcome. Ann Surg 227:566-571

87. Yamamoto J, Sugihara K, Kosuge T et al (1995) Pathologic support for limited hepatectomy in the treatment of liver metastases from colorectal cancer. Ann Surg 221:74-78

88. Elias D, Cavalcanti A, Sabourin JC et al (1998) Results of 136 curative hepatectomies with a safety margin of less than $10 \mathrm{~mm}$ for colorectal metastases. J Surg Oncol 69:88-93

89. Fong Y, Cohen AM, Fortner JG et al (1997) Liver resection for colorectal metastases. J Clin Oncol 15:938-946

90. Kokudo N, Miki Y, Sugai S et al (2002) Genetic and histological assessment of surgical margins in resected liver metastases from colorectal carcinoma: minimum surgical margins for successful resection. Arch Surg 137:833-840

91. Bismuth H, Castaing D, Traynor O (1988) Surgery for synchronous hepatic metastases of colorectal cancer. Scand J Gastroenterol Suppl 149:144-149

92. Scheele J (1993) Hepatectomy for liver metastases. Br J Surg 80:274-276

93. Adam R, de Gramont A, Figueras J et al (2015) Managing synchronous liver metastases from colorectal cancer: a multidisciplinary international consensus. Cancer Treat Rev 41:729-741

94. Rodgers MS, McCall JL (2000) Surgery for colorectal liver metastases with hepatic lymph node involvement: a systematic review. Br J Surg 87:1142-1155

95. Beckurts KT, Holscher AH, Thorban S et al (1997) Significance of lymph node involvement at the hepatic hilum in the resection of colorectal liver metastases. Br J Surg 84:1081-1084

96. Nakamura S, Suzuki S, Konno H (1999) Resection of hepatic metastases of colorectal carcinoma: 20 years' experience. J Hepatobiliary Pancreat Surg 6:16-22 
97. Regnard JF, Grunenwald D, Spaggiari L et al (1998) Surgical treatment of hepatic and pulmonary metastases from colorectal cancers. Ann Thorac Surg 66:214-218 (discussion 218-219)

98. Nordlinger B, Vaillant JC, Guiguet M et al (1994) Survival benefit of repeat liver resections for recurrent colorectal metastases: 143 cases. Association Francaise de Chirurgie. J Clin Oncol 12:1491-1496

99. Yamamoto J, Kosuge T, Shimada K et al (1999) Repeat liver resection for recurrent colorectal liver metastases. Am J Surg 178:275-281

100. Petrowsky H, Gonen M, Jarnagin W et al (2002) Second liver resections are safe and effective treatment for recurrent hepatic metastases from colorectal cancer: a bi-institutional analysis. Ann Surg 235:863-871

101. Suzuki S, Sakaguchi T, Yokoi Y et al (2001) Impact of repeat hepatectomy on recurrent colorectal liver metastases. Surgery 129:421-428

102. Nagakura S, Shirai Y, Suda T et al (2002) Multiple repeat resections of intra- and extrahepatic recurrences in patients undergoing initial hepatectomy for colorectal carcinoma metastases. World J Surg 26:141-147

103. Tanaka K, Shimada H, Ohta M et al (2004) Procedures of choice for resection of primary and recurrent liver metastases from colorectal cancer. World J Surg 28:482-487

104. Thelen A, Jonas S, Benckert C et al (2007) Repeat liver resection for recurrent liver metastases from colorectal cancer. Eur J Surg Oncol 33:324-328

105. Pessaux P, Lermite E, Brehant O et al (2006) Repeat hepatectomy for recurrent colorectal liver metastases. J Surg Oncol 93:1-7

106. Ishiguro S, Akasu T, Fujimoto $Y$ et al (2006) Second hepatectomy for recurrent colorectal liver metastasis: analysis of preoperative prognostic factors. Ann Surg Oncol 13:1579-1587

107. Tree AC, Khoo VS, Eeles RA et al (2013) Stereotactic body radiotherapy for oligometastases. Lancet Oncol 14:e28-e37

108. Goya T, Miyazawa N, Kondo H et al (1989) Surgical resection of pulmonary metastases from colorectal cancer. 10-year follow-up. Cancer 64:1418-1421

109. McCormack PM, Burt ME, Bains MS et al (1992) Lung resection for colorectal metastases. 10-year results. Arch Surg 127:1403-1406

110. Ike H, Shimada H, Ohki S et al (2002) Results of aggressive resection of lung metastases from colorectal carcinoma detected by intensive follow-up. Dis Colon Rectum 45:468-473 (discussion 473-465)

111. Okumura S, Kondo H, Tsuboi M et al (1996) Pulmonary resection for metastatic colorectal cancer: experiences with 159 patients. J Thorac Cardiovasc Surg 112:867-874

112. McAfee MK, Allen MS, Trastek VF et al (1992) Colorectal lung metastases: results of surgical excision. Ann Thorac Surg 53:780-785 (discussion 785-786)

113. Saito Y, Omiya H, Kohno K et al (2002) Pulmonary metastasectomy for 165 patients with colorectal carcinoma: a prognostic assessment. J Thorac Cardiovasc Surg 124:1007-1013

114. Koga R, Yamamoto J, Saiura A et al (2006) Surgical resection of pulmonary metastases from colorectal cancer: four favourable prognostic factors. Jpn J Clin Oncol 36:643-648

115. Iizasa T, Suzuki M, Yoshida S et al (2006) Prediction of prognosis and surgical indications for pulmonary metastasectomy from colorectal cancer. Ann Thorac Surg 82:254-260

116. Watanabe K, Nagai K, Kobayashi A et al (2009) Factors influencing survival after complete resection of pulmonary metastases from colorectal cancer. Br J Surg 96:1058-1065

117. Iida T, Nomori H, Shiba M et al (2013) Prognostic factors after pulmonary metastasectomy for colorectal cancer and rationale for determining surgical indications: a retrospective analysis. Ann Surg 257:1059-1064
118. Kanemitsu Y, Kato T, Hirai T et al (2004) Preoperative probability model for predicting overall survival after resection of pulmonary metastases from colorectal cancer. Br J Surg 91:112-120

119. Hirosawa T, Itabashi M, Ohnuki T et al (2013) Prognostic factors in patients undergoing complete resection of pulmonary metastases of colorectal cancer: a multi-institutional cumulative follow-up study. Surg Today 43:494-499

120. Gonzalez M, Poncet A, Combescure C et al (2013) Risk factors for survival after lung metastasectomy in colorectal cancer patients: a systematic review and meta-analysis. Ann Surg Oncol 20:572-579

121. Miller G, Biernacki P, Kemeny NE et al (2007) Outcomes after resection of synchronous or metachronous hepatic and pulmonary colorectal metastases. J Am Coll Surg 205:231-238

122. Andres A, Mentha G, Adam R et al (2015) Surgical management of patients with colorectal cancer and simultaneous liver and lung metastases. Br J Surg 102:691-699

123. Kandioler D, Kromer E, Tuchler H et al (1998) Long-term results after repeated surgical removal of pulmonary metastases. Ann Thorac Surg 65:909-912

124. Kanzaki R, Higashiyama M, Oda K et al (2011) Outcome of surgical resection for recurrent pulmonary metastasis from colorectal carcinoma. Am J Surg 202:419-426

125. Patchell RA, Tibbs PA, Walsh JW et al (1990) A randomized trial of surgery in the treatment of single metastases to the brain. $\mathrm{N}$ Engl J Med 322:494-500

126. Go PH, Klaassen Z, Meadows MC et al (2011) Gastrointestinal cancer and brain metastasis: a rare and ominous sign. Cancer 117:3630-3640

127. Wronski M, Arbit E (1999) Resection of brain metastases from colorectal carcinoma in 73 patients. Cancer 85:1677-1685

128. D'Andrea G, Isidori A, Caroli E et al (2004) Single cerebral metastasis from colorectal adenocarcinoma. Neurosurg Rev 27:55-57

129. Farnell GF, Buckner JC, Cascino TL et al (1996) Brain metastases from colorectal carcinoma. The long term survivors. Cancer 78:711-716

130. Jung M, Ahn JB, Chang JH et al (2011) Brain metastases from colorectal carcinoma: prognostic factors and outcome. J Neurooncol 101:49-55

131. Aprile G, Zanon E, Tuniz F et al (2009) Neurosurgical management and postoperative whole-brain radiotherapy for colorectal cancer patients with symptomatic brain metastases. J Cancer Res Clin Oncol 135:451-457

132. Noura S, Ohue M, Shingai T et al (2012) Brain metastasis from colorectal cancer: prognostic factors and survival. J Surg Oncol 106:144-148

133. Borgelt B, Gelber R, Kramer S et al (1980) The palliation of brain metastases: final results of the first two studies by the Radiation Therapy Oncology Group. Int J Radiat Oncol Biol Phys 6:1-9

134. Kurtz JM, Gelber R, Brady LW et al (1981) The palliation of brain metastases in a favorable patient population: a randomized clinical trial by the Radiation Therapy Oncology Group. Int J Radiat Oncol Biol Phys 7:891-895

135. Flickinger JC, Kondziolka D, Lunsford LD et al (1994) A multiinstitutional experience with stereotactic radiosurgery for solitary brain metastasis. Int J Radiat Oncol Biol Phys 28:797-802

136. Silva IL, Iskandarani M, Hotouras A et al (2017) A systematic review to assess the management of patients with cerebral metastases secondary to colorectal cancer. Tech Coloproctol $21: 847-852$

137. Gaspar L, Scott C, Rotman M et al (1997) Recursive partitioning analysis (RPA) of prognostic factors in three Radiation Therapy Oncology Group (RTOG) brain metastases trials. Int J Radiat Oncol Biol Phys 37:745-751 
138. van Gijn W, Marijnen CA, Nagtegaal ID et al (2011) Preoperative radiotherapy combined with total mesorectal excision for resectable rectal cancer: 12-year follow-up of the multicentre, randomised controlled TME trial. Lancet Oncol 12:575-582

139. Sperduto PW, Kased N, Roberge D et al (2012) Summary report on the graded prognostic assessment: an accurate and facile diagnosis-specific tool to estimate survival for patients with brain metastases. J Clin Oncol 30:419-425

140. Andrews DW, Scott CB, Sperduto PW et al (2004) Whole brain radiation therapy with or without stereotactic radiosurgery boost for patients with one to three brain metastases: phase III results of the RTOG 9508 randomised trial. Lancet 363:1665-1672

141. Aoyama H, Shirato H, Tago M et al (2006) Stereotactic radiosurgery plus whole-brain radiation therapy vs stereotactic radiosurgery alone for treatment of brain metastases: a randomized controlled trial. JAMA 295:2483-2491

142. Andre T, Boni C, Mounedji-Boudiaf L et al (2004) Oxaliplatin, fluorouracil, and leucovorin as adjuvant treatment for colon cancer. N Engl J Med 350:2343-2351

143. Haller DG, Tabernero J, Maroun J et al (2011) Capecitabine plus oxaliplatin compared with fluorouracil and folinic acid as adjuvant therapy for stage III colon cancer. J Clin Oncol 29:1465-1471

144. Shimada Y, Hamaguchi T, Mizusawa J et al (2014) Randomised phase III trial of adjuvant chemotherapy with oral uracil and tegafur plus leucovorin versus intravenous fluorouracil and levofolinate in patients with stage III colorectal cancer who have undergone Japanese D2/D3 lymph node dissection: final results of JCOG0205. Eur J Cancer 50:2231-2240

145. Yoshida M, Ishiguro M, Ikejiri K et al (2014) S-1 as adjuvant chemotherapy for stage III colon cancer: a randomized phase III study (ACTS-CC trial). Ann Oncol 25:1743-1749

146. Hamaguchi T, Shimada Y, Mizusawa J et al (2018) Capecitabine versus S-1 as adjuvant chemotherapy for patients with stage III colorectal cancer (JCOG0910): an open-label, non-inferiority, randomised, phase 3, multicentre trial. Lancet Gastroenterol Hepatol 3:47-56

147. Grothey A, Sobrero AF, Shields AF et al (2018) Duration of Adjuvant Chemotherapy for Stage III Colon Cancer. N Engl J Med 378:1177-1188

148. Yoshino T, Yamanaka T, Kotaka D et al (2017) Efficacy of 3 versus 6 months of oxaliplatin-based adjuvant chemotherapy for stage III colon cancer (CC): results from phase III ACHIEVE trial as part of the International Duration Evaluation of Adjuvant therapy (IDEA) Collaboration. An Oncol 28(mdx440):017

149. Kotaka M, Yamanaka T, Yoshino T et al (2018) Safety data from the phase III Japanese ACHIEVE trial: part of an international, prospective, planned pooled analysis of six phase III trials comparing 3 versus 6 months of oxaliplatin-based adjuvant chemotherapy for stage III colon cancer. ESMO Open 3:e000354

150. Sadahiro S, Tsuchiya T, Sasaki K et al (2015) Randomized phase III trial of treatment duration for oral uracil and tegafur plus leucovorin as adjuvant chemotherapy for patients with stage IIB/III colon cancer: final results of JFMC33-0502. Ann Oncol 26:2274-2280

151. Yamaguchi S, Kunieda K, Sato T et al (2016) Phase III trial of 24 weeks vs. 48 weeks capecitabine adjuvant chemotherapy for patients with stage III colon cancer: final results of JFMC370801. Ann Oncol 27(suppl 6):469PD

152. Sakamoto J, Hamada C, Yoshida S et al (2007) An individual patient data meta-analysis of adjuvant therapy with uracil-tegafur (UFT) in patients with curatively resected rectal cancer. $\mathrm{Br} \mathbf{J}$ Cancer 96:1170-1177

153. Oki E, Murata A, Yoshida K et al (2016) A randomized phase III trial comparing S-1 versus UFT as adjuvant chemotherapy for stage II/III rectal cancer (JFMC35-C1: ACTS-RC). Ann Oncol 27:1266-1272

154. Matsuda $C$, Ishiguro $M$, Teramukai $S$ et al (2018) A randomisedcontrolled trial of 1-year adjuvant chemotherapy with oral tegafur-uracil versus surgery alone in stage II colon cancer: SACURA trial. Eur J Cancer 96:54-63

155. Hasegawa K, Saiura A, Takayama T et al (2016) Adjuvant oral uracil-tegafur with leucovorin for colorectal cancer liver metastases: a randomized controlled trial. PLoS ONE 11:e0162400

156. Simmonds PC (2000) Palliative chemotherapy for advanced colorectal cancer: systematic review and meta-analysis. Colorectal Cancer Collaborative Group. BMJ 321:531-535

157. Yamada Y, Takahari D, Matsumoto H et al (2013) Leucovorin, fluorouracil, and oxaliplatin plus bevacizumab versus $\mathrm{S}-1$ and oxaliplatin plus bevacizumab in patients with metastatic colorectal cancer (SOFT): an open-label, non-inferiority, randomised phase 3 trial. Lancet Oncol 14:1278-1286

158. Loupakis F, Cremolini C, Masi G et al (2014) Initial therapy with FOLFOXIRI and bevacizumab for metastatic colorectal cancer. N Engl J Med 371:1609-1618

159. Yamazaki K, Nagase M, Tamagawa $H$ et al (2016) Randomized phase III study of bevacizumab plus FOLFIRI and bevacizumab plus mFOLFOX6 as first-line treatment for patients with metastatic colorectal cancer (WJOG4407G). Ann Oncol 27:1539-1546

160. Cunningham D, Pyrhonen S, James RD et al (1998) Randomised trial of irinotecan plus supportive care versus supportive care alone after fluorouracil failure for patients with metastatic colorectal cancer. Lancet 352:1413-1418

161. Taniguchi H, Yamazaki K, Yoshino T et al (2015) Japanese Society of Medical Oncology Clinical Guidelines: RAS (KRAS/ NRAS) mutation testing in colorectal cancer patients. Cancer Sci 106:324-327

162. de Gramont A, Figer A, Seymour M et al (2000) Leucovorin and fluorouracil with or without oxaliplatin as first-line treatment in advanced colorectal cancer. J Clin Oncol 18:2938-2947

163. Goldberg RM, Sargent DJ, Morton RF et al (2004) A randomized controlled trial of fluorouracil plus leucovorin, irinotecan, and oxaliplatin combinations in patients with previously untreated metastatic colorectal cancer. J Clin Oncol 22:23-30

164. Saltz LB, Clarke S, Diaz-Rubio E et al (2008) Bevacizumab in combination with oxaliplatin-based chemotherapy as first-line therapy in metastatic colorectal cancer: a randomized phase III study. J Clin Oncol 26:2013-2019

165. Cassidy J, Clarke S, Diaz-Rubio E et al (2008) Randomized phase III study of capecitabine plus oxaliplatin compared with fluorouracil/folinic acid plus oxaliplatin as first-line therapy for metastatic colorectal cancer. J Clin Oncol 26:2006-2012

166. Tournigand C, Andre T, Achille E et al (2004) FOLFIRI followed by FOLFOX6 or the reverse sequence in advanced colorectal cancer: a randomized GERCOR study. J Clin Oncol 22:229-237

167. Douillard JY, Cunningham D, Roth AD et al (2000) Irinotecan combined with fluorouracil compared with fluorouracil alone as first-line treatment for metastatic colorectal cancer: a multicentre randomised trial. Lancet 355:1041-1047

168. Fuchs CS, Marshall J, Mitchell E et al (2007) Randomized, controlled trial of irinotecan plus infusional, bolus, or oral fluoropyrimidines in first-line treatment of metastatic colorectal cancer: results from the BICC-C Study. J Clin Oncol 25:4779-4786

169. Yamada Y, Denda T, Gamoh M et al (2018) S-1 and irinotecan plus bevacizumab versus mFOLFOX6 or CapeOX plus bevacizumab as first-line treatment in patients with metastatic colorectal cancer (TRICOLORE): a randomized, open-label, phase III, noninferiority trial. Ann Oncol 29:624-631 
170. Bokemeyer C, Bondarenko I, Makhson A et al (2009) Fluorouracil, leucovorin, and oxaliplatin with and without cetuximab in the first-line treatment of metastatic colorectal cancer. J Clin Oncol 27:663-671

171. Douillard JY, Siena S, Cassidy J et al (2010) Randomized, phase III trial of panitumumab with infusional fluorouracil, leucovorin, and oxaliplatin (FOLFOX4) versus FOLFOX4 alone as first-line treatment in patients with previously untreated metastatic colorectal cancer: the PRIME study. J Clin Oncol 28:4697-4705

172. Van Cutsem E, Kohne CH, Hitre E et al (2009) Cetuximab and chemotherapy as initial treatment for metastatic colorectal cancer. N Engl J Med 360:1408-1417

173. Kohne CH, Hofheinz R, Mineur L et al (2012) First-line panitumumab plus irinotecan/5-fluorouracil/leucovorin treatment in patients with metastatic colorectal cancer. J Cancer Res Clin Oncol 138:65-72

174. Falcone A, Ricci S, Brunetti I et al (2007) Phase III trial of infusional fluorouracil, leucovorin, oxaliplatin, and irinotecan (FOLFOXIRI) compared with infusional fluorouracil, leucovorin, and irinotecan (FOLFIRI) as first-line treatment for metastatic colorectal cancer: the Gruppo Oncologico Nord Ovest. J Clin Oncol 25:1670-1676

175. Cremolini C, Loupakis F, Antoniotti C et al (2015) FOLFOXIRI plus bevacizumab versus FOLFIRI plus bevacizumab as first-line treatment of patients with metastatic colorectal cancer: updated overall survival and molecular subgroup analyses of the openlabel, phase 3 TRIBE study. Lancet Oncol 16:1306-1315

176. Petrelli N, Herrera L, Rustum Y et al (1987) A prospective randomized trial of 5-fluorouracil versus 5-fluorouracil and highdose leucovorin versus 5-fluorouracil and methotrexate in previously untreated patients with advanced colorectal carcinoma. J Clin Oncol 5:1559-1565

177. de Gramont A, Bosset JF, Milan C et al (1997) Randomized trial comparing monthly low-dose leucovorin and fluorouracil bolus with bimonthly high-dose leucovorin and fluorouracil bolus plus continuous infusion for advanced colorectal cancer: a French intergroup study. J Clin Oncol 15:808-815

178. Hurwitz HI, Fehrenbacher L, Hainsworth JD et al (2005) Bevacizumab in combination with fluorouracil and leucovorin: an active regimen for first-line metastatic colorectal cancer. J Clin Oncol 23:3502-3508

179. Kabbinavar F, Hurwitz HI, Fehrenbacher L et al (2003) Phase II, randomized trial comparing bevacizumab plus fluorouracil (FU)/ leucovorin (LV) with FU/LV alone in patients with metastatic colorectal cancer. J Clin Oncol 21:60-65

180. Hoff PM, Ansari R, Batist G et al (2001) Comparison of oral capecitabine versus intravenous fluorouracil plus leucovorin as first-line treatment in 605 patients with metastatic colorectal cancer: results of a randomized phase III study. J Clin Oncol 19:2282-2292

181. Van Cutsem E, Twelves C, Cassidy J et al (2001) Oral capecitabine compared with intravenous fluorouracil plus leucovorin in patients with metastatic colorectal cancer: results of a large phase III study. J Clin Oncol 19:4097-4106

182. Tebbutt NC, Wilson K, Gebski VJ et al (2010) Capecitabine, bevacizumab, and mitomycin in first-line treatment of metastatic colorectal cancer: results of the Australasian Gastrointestinal Trials Group Randomized Phase III MAX Study. J Clin Oncol 28:3191-3198

183. Shirao K, Hoff PM, Ohtsu A et al (2004) Comparison of the efficacy, toxicity, and pharmacokinetics of a uracil/tegafur (UFT) plus oral leucovorin (LV) regimen between Japanese and American patients with advanced colorectal cancer: joint United States and Japan study of UFT/LV. J Clin Oncol 22:3466-3474

184. Douillard JY, Hoff PM, Skillings JR et al (2002) Multicenter phase III study of uracil/tegafur and oral leucovorin versus fluorouracil and leucovorin in patients with previously untreated metastatic colorectal cancer. J Clin Oncol 20:3605-3616

185. Carmichael J, Popiela T, Radstone D et al (2002) Randomized comparative study of tegafur/uracil and oral leucovorin versus parenteral fluorouracil and leucovorin in patients with previously untreated metastatic colorectal cancer. J Clin Oncol 20:3617-3627

186. Nishina T, Moriwaki T, Shimada M et al (2016) Uracil-tegafur and oral leucovorin combined with bevacizumab in elderly patients (aged $>/=75$ years) with metastatic colorectal cancer: a multicenter, phase II trial (Joint study of bevacizumab, oral leucovorin, and uracil-tegafur in elderly patients [J-BLUE] study). Clin Colorectal Cancer 15:236-242

187. Yoshida M, Muro K, Tsuji A et al (2015) Combination chemotherapy with bevacizumab and S-1 for elderly patients with metastatic colorectal cancer (BASIC trial). Eur J Cancer 51:935-941

188. Pietrantonio F, Cremolini C, Aprile G et al (2015) Single-agent panitumumab in frail elderly patients with advanced RAS and BRAF wild-type colorectal cancer: challenging drug label to light up new hope. Oncologist 20:1261-1265

189. Sastre J, Massuti B, Pulido G et al (2015) First-line single-agent panitumumab in frail elderly patients with wild-type KRAS metastatic colorectal cancer and poor prognostic factors: a phase II study of the Spanish Cooperative Group for the Treatment of Digestive Tumours. Eur J Cancer 51:1371-1380

190. Bennouna J, Sastre J, Arnold D et al (2013) Continuation of bevacizumab after fi progression in metastatic colorectal cancer (ML18147): a randomised phase 3 trial. Lancet Oncol 14:29-37

191. Iwamoto $\mathrm{S}$, Takahashi T, Tamagawa $\mathrm{H}$ et al (2015) FOLFIRI plus bevacizumab as second-line therapy in patients with metastatic colorectal cancer after first-line bevacizumab plus oxaliplatinbased therapy: the randomized phase III EAGLE study. Ann Oncol 26:1427-1433

192. Xu RH, Muro K, Morita S et al (2018) Modified XELIRI (capecitabine plus irinotecan) versus FOLFIRI (leucovorin, fluorouracil, and irinotecan), both either with or without bevacizumab, as second-line therapy for metastatic colorectal cancer (AXEPT): a multicentre, open-label, randomised, non-inferiority, phase 3 trial. Lancet Oncol 19:660-671

193. Tabernero J, Yoshino T, Cohn AL et al (2015) Ramucirumab versus placebo in combination with second-line FOLFIRI in patients with metastatic colorectal carcinoma that progressed during or after first-line therapy with bevacizumab, oxaliplatin, and a fluoropyrimidine (RAISE): a randomised, double-blind, multicentre, phase 3 study. Lancet Oncol 16:499-508

194. Van Cutsem E, Tabernero J, Lakomy R et al (2012) Addition of aflibercept to fluorouracil, leucovorin, and irinotecan improves survival in a phase III randomized trial in patients with metastatic colorectal cancer previously treated with an oxaliplatinbased regimen. J Clin Oncol 30:3499-3506

195. Muro K, Boku N, Shimada Y et al (2010) Irinotecan plus S-1 (IRIS) versus fluorouracil and folinic acid plus irinotecan (FOLFIRI) as second-line chemotherapy for metastatic colorectal cancer: a randomised phase $2 / 3$ non-inferiority study (FIRIS study). Lancet Oncol 11:853-860

196. Rougier P, Van Cutsem E, Bajetta E et al (1998) Randomised trial of irinotecan versus fluorouracil by continuous infusion after fluorouracil failure in patients with metastatic colorectal cancer. Lancet 352:1407-1412

197. Kuramochi H, Ando M, Itabashi M et al (2017) Phase II study of bevacizumab and irinotecan as second-line therapy for patients with metastatic colorectal cancer previously treated with fluoropyrimidines, oxaliplatin, and bevacizumab. Cancer Chemother Pharmacol 79:579-585

198. Peeters M, Price TJ, Cervantes A et al (2010) Randomized phase III study of panitumumab with fluorouracil, leucovorin, and 
irinotecan (FOLFIRI) compared with FOLFIRI alone as secondline treatment in patients with metastatic colorectal cancer. J Clin Oncol 28:4706-4713

199. Shitara K, Yonesaka K, Denda T et al (2016) Randomized study of FOLFIRI plus either panitumumab or bevacizumab for wild-type KRAS colorectal cancer-WJOG 6210G. Cancer Sci 107:1843-1850

200. Sobrero AF, Maurel J, Fehrenbacher L et al (2008) EPIC: phase III trial of cetuximab plus irinotecan after fluoropyrimidine and oxaliplatin failure in patients with metastatic colorectal cancer. J Clin Oncol 26:2311-2319

201. Seymour MT, Brown SR, Middleton G et al (2013) Panitumumab and irinotecan versus irinotecan alone for patients with KRAS wild-type, fluorouracil-resistant advanced colorectal cancer (PICCOLO): a prospectively stratified randomised trial. Lancet Oncol 14:749-759

202. Le DT, Uram JN, Wang H et al (2015) PD-1 Blockade in Tumors with Mismatch-Repair Deficiency. N Engl J Med 372:2509-2520

203. Rothenberg ML, Oza AM, Bigelow RH et al (2003) Superiority of oxaliplatin and fluorouracil-leucovorin compared with either therapy alone in patients with progressive colorectal cancer after irinotecan and fluorouracil-leucovorin: interim results of a phase III trial. J Clin Oncol 21:2059-2069

204. Giantonio BJ, Catalano PJ, Meropol NJ et al (2007) Bevacizumab in combination with oxaliplatin, fluorouracil, and leucovorin (FOLFOX4) for previously treated metastatic colorectal cancer: results from the Eastern Cooperative Oncology Group Study E3200. J Clin Oncol 25:1539-1544

205. Rothenberg ML, Cox JV, Butts C et al (2008) Capecitabine plus oxaliplatin (XELOX) versus 5-fluorouracil/folinic acid plus oxaliplatin (FOLFOX-4) as second-line therapy in metastatic colorectal cancer: a randomized phase III noninferiority study. Ann Oncol 19:1720-1726

206. Cunningham D, Humblet Y, Siena S et al (2004) Cetuximab monotherapy and cetuximab plus irinotecan in irinotecan-refractory metastatic colorectal cancer. N Engl J Med 351:337-345

207. Jonker DJ, O'Callaghan CJ, Karapetis CS et al (2007) Cetuximab for the treatment of colorectal cancer. N Engl J Med 357:2040-2048

208. Karapetis CS, Khambata-Ford S, Jonker DJ et al (2008) K-ras mutations and benefit from cetuximab in advanced colorectal cancer. N Engl J Med 359:1757-1765

209. Van Cutsem E, Peeters M, Siena S et al (2007) Open-label phase III trial of panitumumab plus best supportive care compared with best supportive care alone in patients with chemotherapy-refractory metastatic colorectal cancer. J Clin Oncol 25:1658-1664

210. Amado RG, Wolf M, Peeters M et al (2008) Wild-type KRAS is required for panitumumab efficacy in patients with metastatic colorectal cancer. J Clin Oncol 26:1626-1634

211. Grothey A, Van Cutsem E, Sobrero A et al (2013) Regorafenib monotherapy for previously treated metastatic colorectal cancer (CORRECT): an international, multicentre, randomised, placebo-controlled, phase 3 trial. Lancet 381:303-312

212. Yoshino T, Mizunuma N, Yamazaki K et al (2012) TAS-102 monotherapy for pretreated metastatic colorectal cancer: a double-blind, randomised, placebo-controlled phase 2 trial. Lancet Oncol 13:993-1001

213. Mayer RJ, Van Cutsem E, Falcone A et al (2015) Randomized trial of TAS-102 for refractory metastatic colorectal cancer. N Engl J Med 372:1909-1919

214. Xu J, Kim TW, Shen L et al (2018) Results of a randomized, double-blind, placebo-controlled, phase III trial of trifluridine/ tipiracil (TAS-102) monotherapy in Asian patients with previously treated metastatic colorectal cancer: the TERRA Study. J Clin Oncol 36:350-358
215. Satoh T, Ura T, Yamada Y et al (2011) Genotype-directed, dosefinding study of irinotecan in cancer patients with UGT1A $1 * 28$ and/or UGT1A1*6 polymorphisms. Cancer Sci 102:1868-1873

216. Oki E, Kato T, Bando H et al (2018) A multicenter clinical phase II study of FOLFOXIRI plus bevacizumab as first-line therapy in patients with metastatic colorectal cancer: QUATTRO Study. Clin Colorectal Cancer 17:147-155

217. Meta-Analysis Group in C, Piedbois P, Buyse M et al (1996) Reappraisal of hepatic arterial infusion in the treatment of nonresectable liver metastases from colorectal cancer. J Natl Cancer Inst 88:252-258

218. Yoshino T, Arnold D, Taniguchi H et al (2018) Pan-Asian adapted ESMO consensus guidelines for the management of patients with metastatic colorectal cancer: a JSMO-ESMO initiative endorsed by CSCO, KACO, MOS, SSO and TOS. Ann Oncol 29:44-70

219. Yamazaki K, Taniguchi H, Yoshino T et al (2018) Japanese Society of medical oncology clinical guidelines: molecular testing for colorectal cancer treatment, third edition. Cancer Sci 109:2074-2079

220. Arnold D, Lueza B, Douillard JY et al (2017) Prognostic and predictive value of primary tumour side in patients with RAS wildtype metastatic colorectal cancer treated with chemotherapy and EGFR directed antibodies in six randomized trials. Ann Oncol 28:1713-1729

221. Yokota T, Ura T, Shibata $\mathrm{N}$ et al (2011) BRAF mutation is a powerful prognostic factor in advanced and recurrent colorectal cancer. Br J Cancer 104:856-862

222. Kawazoe A, Shitara K, Fukuoka S et al (2015) A retrospective observational study of clinicopathological features of KRAS, NRAS, BRAF and PIK3CA mutations in Japanese patients with metastatic colorectal cancer. BMC Cancer 15:258

223. Loupakis F, Cremolini C, Salvatore L et al (2014) FOLFOXIRI plus bevacizumab as first-line treatment in BRAF mutant metastatic colorectal cancer. Eur J Cancer 50:57-63

224. van Geel R, Tabernero J, Elez E et al (2017) A phase Ib Doseescalation study of encorafenib and cetuximab with or without alpelisib in metastatic BRAF-mutant colorectal cancer. Cancer Discov 7:610-619

225. Corcoran RB, Andre T, Atreya CE et al (2018) Combined BRAF, EGFR, and MEK inhibition in patients with BRAF(V600E)mutant colorectal cancer. Cancer Discov 8:428-443

226. Overman MJ, McDermott R, Leach JL et al (2017) Nivolumab in patients with metastatic DNA mismatch repair-deficient or microsatellite instability-high colorectal cancer (CheckMate 142): an open-label, multicentre, phase 2 study. Lancet Oncol 18:1182-1191

227. Ishida $H$, Yamaguchi $T$, Tanakaya $K$ et al (2018) Japanese society for cancer of the colon and rectum (JSCCR) guidelines 2016 for the clinical practice of hereditary colorectal cancer (translated version). J Anus Rectum Colon 2:1-51

228. Skibber JMHP, Minsky BD (2001) Cancer of the rectum. In: Devita VT, Hellman S, Rosenberg SA (eds) Cancer: principles and practice of oncology, 6th edn. Lippincott, Williams and Wilkins, Philadelphia, pp 1271-1318

229. Swedish Rectal Cancer Trial (1997) Improved survival with preoperative radiotherapy in resectable rectal cancer. N Engl J Med 336:980-987

230. Camma C, Giunta M, Fiorica F et al (2000) Preoperative radiotherapy for resectable rectal cancer: a meta-analysis. JAMA 284:1008-1015

231. Adjuvant radiotherapy for rectal cancer (2001) a systematic overview of 8,507 patients from 22 randomised trials. Lancet 358:1291-1304

232. Kapiteijn E, Marijnen CA, Nagtegaal ID et al (2001) Preoperative radiotherapy combined with total mesorectal excision for resectable rectal cancer. N Engl J Med 345:638-646 
233. Peeters KC, Marijnen CA, Nagtegaal ID et al (2007) The TME trial after a median follow-up of 6 years: increased local control but no survival benefit in irradiated patients with resectable rectal carcinoma. Ann Surg 246:693-701

234. Marijnen CA, van de Velde CJ, Putter H et al (2005) Impact of short-term preoperative radiotherapy on health-related quality of life and sexual functioning in primary rectal cancer: report of a multicenter randomized trial. J Clin Oncol 23:1847-1858

235. Peeters KC, van de Velde CJ, Leer JW et al (2005) Late side effects of short-course preoperative radiotherapy combined with total mesorectal excision for rectal cancer: increased bowel dysfunction in irradiated patients-a Dutch colorectal cancer group study. J Clin Oncol 23:6199-6206

236. Francois Y, Nemoz CJ, Baulieux J et al (1999) Infl of the interval between preoperative radiation therapy and surgery on downstaging and on the rate of sphincter-sparing surgery for rectal cancer: the Lyon R90-01 randomized trial. J Clin Oncol 17:2396

237. Bosset JF, Collette L, Calais G et al (2006) Chemotherapy with preoperative radiotherapy in rectal cancer. N Engl J Med 355:1114-1123

238. Gerard JP, Conroy T, Bonnetain F et al (2006) Preoperative radiotherapy with or without concurrent fluorouracil and leucovorin in T3-4 rectal cancers: results of FFCD 9203. J Clin Oncol 24:4620-4625

239. Bujko K, Nowacki MP, Nasierowska-Guttmejer A et al (2006) Long-term results of a randomized trial comparing preoperative short-course radiotherapy with preoperative conventionally fractionated chemoradiation for rectal cancer. Br J Surg 93:1215-1223

240. Ngan SY, Burmeister B, Fisher RJ et al (2012) Randomized trial of short-course radiotherapy versus long-course chemoradiation comparing rates of local recurrence in patients with T3 rectal cancer: trans-Tasman Radiation Oncology Group trial 01.04. J Clin Oncol 30:3827-3833

241. Sauer R, Becker H, Hohenberger W et al (2004) Preoperative versus postoperative chemoradiotherapy for rectal cancer. $\mathrm{N}$ Engl J Med 351:1731-1740

242. Hofheinz RD, Wenz F, Post S et al (2012) Chemoradiotherapy with capecitabine versus fluorouracil for locally advanced rectal cancer: a randomised, multicentre, non-inferiority, phase 3 trial. Lancet Oncol 13:579-588

243. Allegra CJ, Yothers G, O'Connell MJ et al (2015) Neoadjuvant 5-FU or capecitabine plus radiation with or without oxaliplatin in rectal cancer patients: A phase III randomized clinical trial. J Natl Cancer Inst 2015:107

244. Aschele C, Cionini L, Lonardi S et al (2011) Primary tumor response to preoperative chemoradiation with or without oxaliplatin in locally advanced rectal cancer: pathologic results of the STAR-01 randomized phase III trial. J Clin Oncol 29:2773-2780

245. Gerard JP, Azria D, Gourgou-Bourgade S et al (2010) Comparison of two neoadjuvant chemoradiotherapy regimens for locally advanced rectal cancer: results of the phase III trial ACCORD 12/0405-Prodige 2. J Clin Oncol 28:1638-1644

246. Gerard JP, Azria D, Gourgou-Bourgade S (2012) Clinical outcome of the ACCORD 12/0405 PRODIGE 2 randomized trial in rectal cancer. J Clin Oncol 30:4558-4565

247. Rodel C, Liersch T, Becker H et al (2012) Preoperative chemoradiotherapy and postoperative chemotherapy with fluorouracil and oxaliplatin versus fluorouracil alone in locally advanced rectal cancer: initial results of the German CAO/ARO/AIO-04 randomised phase 3 trial. Lancet Oncol 13:679-687

248. Cancer pain relief and palliative care (1990) Report of a WHO expert committee. In: WHO Technical Report Series 804. WHO, Geneva, pp 21-22
249. van der Stok EP, Spaander MCW, Grunhagen DJ et al (2017) Surveillance after curative treatment for colorectal cancer. Nat Rev Clin Oncol 14:297-315

250. Meyerhardt JA, Mangu PB, Flynn PJ et al (2013) Follow-up care, surveillance protocol, and secondary prevention measures for survivors of colorectal cancer: American Society of Clinical Oncology clinical practice guideline endorsement. J Clin Oncol 31:4465-4470

251. National Comprehensive Cancer Network (2018) NCCN clinical practice guidelines in oncology-colon cancer, version 3, 2018. http://www.nccn.org/professionals/physician_gls/pdf/colon.pdf. Accessed 7 Aug 2018

252. Labianca R, Nordlinger B, Beretta GD et al (2013) Early colon cancer: ESMO Clinical Practice Guidelines for diagnosis, treatment and follow-up. Ann Oncol 24:vi64-vi72

253. Desch CE, Benson AB 3rd, Somerfield MR et al (2005) American Society of Clinical Oncology: colorectal cancer surveillance: 2005 update of an American Society of Clinical Oncology Practice Guideline. J Clin Oncol 23:8512-8519

254. Steele SR, Chang GJ, Hendren S et al (2015) Practice guideline for the surveillance of patients after curative treatment of colon and rectal cancer. Dis Colon Rectum 58:713-725

255. Green RJ, Metlay JP, Propert K et al (2002) Surveillance for second primary colorectal cancer after adjuvant chemotherapy: an analysis of Intergroup 0089. Ann Intern Med 136:261-269

256. Berman JM, Cheung RJ, Weinberg DS (2000) Surveillance after colorectal cancer resection. Lancet 355:395-399

257. Japanese Society for Cancer of the Colon and Rectum (2016) JSCCR guidelines 2016 for the clinical practice of hereditary colorectal cancer. Kanehara \& CO., LTD. Tokyo

258. Ueno H, Mochizuki H, Hashiguchi Y et al (2004) Risk factors for an adverse outcome in early invasive colorectal carcinoma. Gastroenterology 127:385-394

259. Kitajima K, Fujimori T, Fujii S et al (2004) Correlations between lymph node metastasis and depth of submucosal invasion in submucosal invasive colorectal carcinoma: a Japanese collaborative study. J Gastroenterol 39:534-543

260. Tanaka S, Haruma K, Oh EH et al (2000) Conditions of curability after endoscopic resection for colorectal carcinoma with submucosally massive invasion. Oncol Rep 7:783-788

261. Japanese Society for Cancer of the Colon and Rectum (1980) General rules for clinical and pathological studies on cancer of the colon, rectum and anus, 2nd edn. Kanehara \& CO., LTD., Tokyo

262. Japanese Society for Cancer of the Colon and Rectum (1994) General rules for clinical and pathological studies on cancer of the colon, rectum and anus, 5th edn. Kanehara \& CO., LTD., Tokyo

263. Japanese Society for Cancer of the Colon and Rectum (2005) JSCCR guidelines 2005 for the treatment of colorectal cancer. Kanehara \& CO., LTD., Tokyo

264. Japanese Society for Cancer of the Colon and Rectum (2009) JSCCR guidelines 2009 for the treatment of colorectal cancer. Kanehara \& CO., LTD., Tokyo

265. Ikematsu H, Yoda Y, Matsuda T et al (2013) Long-term outcomes after resection for submucosal invasive colorectal cancers. Gastroenterology 144:551-559 (quiz e14)

266. Matsuda T, Fukuzawa M, Uraoka T et al (2011) Risk of lymph node metastasis in patients with pedunculated type early invasive colorectal cancer: a retrospective multicenter study. Cancer Sci 102:1693-1697

267. Tamaru Y, Oka S, Tanaka S et al (2017) Long-term outcomes after treatment for $\mathrm{T} 1$ colorectal carcinoma: a multicenter retrospective cohort study of Hiroshima GI Endoscopy Research Group. J Gastroenterol 52:1169-1179 
268. Pimentel-Nunes P, Dinis-Ribeiro M, Ponchon T et al (2015) Endoscopic submucosal dissection: European Society of Gastrointestinal Endoscopy (ESGE) Guideline. Endoscopy 47:829-854

269. Takeuchi Y, Iishi H, Tanaka S et al (2014) Factors associated with technical difficulties and adverse events of colorectal endoscopic submucosal dissection: retrospective exploratory factor analysis of a multicenter prospective cohort. Int J Colorectal Dis 29:1275-1284

270. Wada Y, Kudo SE, Tanaka S et al (2015) Predictive factors for complications in endoscopic resection of large colorectal lesions: a multicenter prospective study. Surg Endosc 29:1216-1222

271. Nakajima T, Saito Y, Tanaka S et al (2013) Current status of endoscopic resection strategy for large, early colorectal neoplasia in Japan. Surg Endosc 27:3262-3270

272. Saito Y, Uraoka T, Yamaguchi Y et al (2010) A prospective, multicenter study of 1111 colorectal endoscopic submucosal dissections (with video). Gastrointest Endosc 72(6):1217-1225

273. Hojo K, Koyama Y, Moriya Y (1982) Lymphatic spread and its prognostic value in patients with rectal cancer. Am J Surg 144:350-354

274. Moriya Y, Hojo K, Sawada T et al (1989) Significance of lateral node dissection for advanced rectal carcinoma at or below the peritoneal reflection. Dis Colon Rectum 32:307-315

275. Sugihara K, Moriya Y, Akasu T et al (1996) Pelvic autonomic nerve preservation for patients with rectal carcinoma. Oncologic and functional outcome. Cancer 78:1871-1880

276. Takahashi T, Ueno M, Azekura K et al (2000) Lateral node dissection and total mesorectal excision for rectal cancer. Dis Colon Rectum 43:S59-S68

277. Mori T, Takahashi K, Yasuno M (1998) Radical resection with autonomic nerve preservation and lymph node dissection techniques in lower rectal cancer surgery and its results: the impact of lateral lymph node dissection. Langenbecks Arch Surg 383:409-415

278. Ueno H, Mochizuki H, Hashiguchi Y et al (2001) Prognostic determinants of patients with lateral nodal involvement by rectal cancer. Ann Surg 234:190-197

279. Ueno M, Oya M, Azekura K et al (2005) Incidence and prognostic significance of lateral lymph node metastasis in patients with advanced low rectal cancer. Br J Surg 92:756-763

280. Kanemitsu Y, Komori K, Shida D et al (2017) Potential impact of lateral lymph node dissection (LLND) for low rectal cancer on prognoses and local control: a comparison of 2 high-volume centers in Japan that employ different policies concerning LLND. Surgery 162:303-314

281. Ueno H, Mochizuki H, Hashiguchi Y et al (2007) Potential prognostic benefit of lateral pelvic node dissection for rectal cancer located below the peritoneal reflection. Ann Surg 245:80-87

282. Akiyoshi T, Watanabe T, Miyata S et al (2012) Results of a Japanese nationwide multi-institutional study on lateral pelvic lymph node metastasis in low rectal cancer: is it regional or distant disease? Ann Surg 255:1129-1134

283. Ozawa H, Kotake K, Hosaka M et al (2016) Impact of Lateral Pelvic Lymph Node Dissection on the Survival of Patients with T3 and T4 Low Rectal Cancer. World J Surg 40:1492-1499

284. Oh HK, Kang SB, Lee SM et al (2014) Neoadjuvant chemoradiotherapy affects the indications for lateral pelvic node dissection in mid/low rectal cancer with clinically suspected lateral node involvement: a multicenter retrospective cohort study. Ann Surg Oncol 21:2280-2287

285. Akiyoshi T, Matsueda K, Hiratsuka M et al (2015) Indications for Lateral Pelvic Lymph Node Dissection Based on Magnetic Resonance Imaging Before and After Preoperative Chemoradiotherapy in Patients with Advanced Low-Rectal Cancer. Ann Surg Oncol 22(Suppl 3):S614-S620

286. Fujita S, Mizusawa J, Kanemitsu Y et al (2017) Mesorectal excision with or without lateral lymph node dissection for clinical stage II/III lower rectal cancer (JCOG0212): a multicenter, randomized controlled, noninferiority trial. Ann Surg 266:201-207

287. Pestieau SR, Sugarbaker PH (2000) Treatment of primary colon cancer with peritoneal carcinomatosis: comparison of concomitant vs. delayed management. Dis Colon Rectum 43:1341-1346 (discussion 1347-1348 )

288. Ozawa H, Kotake K, Kobayashi H et al (2014) Prognostic factors for peritoneal carcinomatosis originating from colorectal cancer: an analysis of 921 patients from a multi-institutional database. Surg Today 44:1643-1650

289. Shida D, Tsukamoto S, Ochiai H et al (2018) Long-term outcomes after R0 resection of synchronous peritoneal metastasis from colorectal cancer without cytoreductive surgery or hyperthermic intraperitoneal chemotherapy. Ann Surg Oncol 25:173-178

290. Shida D, Yoshida T, Tanabe T et al (2018) Prognostic impact of R0 resection and targeted therapy for colorectal cancer with synchronous peritoneal metastasis. Ann Surg Oncol 25:1646-1653

291. Kobayashi H, Kotake K, Funahashi K et al (2014) Clinical benefit of surgery for stage IV colorectal cancer with synchronous peritoneal metastasis. J Gastroenterol 49:646-654

292. Kobayashi H, Kotake K, Sugihara K (2014) Outcomes of surgery without HIPEC for synchronous peritoneal metastasis from colorectal cancer: data from a multi-center registry. Int J Clin Oncol 19:98-105

293. Adam R, Wicherts DA, de Haas RJ et al (2008) Complete pathologic response after preoperative chemotherapy for colorectal liver metastases: myth or reality? J Clin Oncol 26:1635-1641

294. van Vledder MG, de Jong MC, Pawlik TM et al (2010) Disappearing colorectal liver metastases after chemotherapy: should we be concerned? J Gastrointest Surg 14:1691-1700

295. Auer RC, White RR, Kemeny NE et al (2010) Predictors of a true complete response among disappearing liver metastases from colorectal cancer after chemotherapy. Cancer 116:1502-1509

296. Benoist S, Brouquet A, Penna C et al (2006) Complete response of colorectal liver metastases after chemotherapy: does it mean cure? J Clin Oncol 24:3939-3945

297. Allen PJ, Kemeny N, Jarnagin W et al (2003) Importance of response to neoadjuvant chemotherapy in patients undergoing resection of synchronous colorectal liver metastases. J Gastrointest Surg 7:109-117

298. Kuebler JP, Wieand HS, O'Connell MJ et al (2007) Oxaliplatin combined with weekly bolus fluorouracil and leucovorin as surgical adjuvant chemotherapy for stage II and III colon cancer: results from NSABP C-07. J Clin Oncol 25:2198-2204

299. Andre T, Boni C, Navarro M et al (2009) Improved overall survival with oxaliplatin, fluorouracil, and leucovorin as adjuvant treatment in stage II or III colon cancer in the MOSAIC trial. J Clin Oncol 27:3109-3116

300. Yothers G, O'Connell MJ, Allegra CJ et al (2011) Oxaliplatin as adjuvant therapy for colon cancer: updated results of NSABP C-07 trial, including survival and subset analyses. J Clin Oncol 29:3768-3774

301. Efficacy of adjuvant fluorouracil and folinic acid in colon cancer (1995) International Multicentre Pooled Analysis of Colon Cancer Trials (IMPACT) investigators. Lancet 345:939-944

302. Twelves C, Wong A, Nowacki MP et al (2005) Capecitabine as adjuvant treatment for stage III colon cancer. N Engl J Med 352:2696-2704

303. Lembersky BC, Wieand HS, Petrelli NJ et al (2006) Oral uracil and tegafur plus leucovorin compared with intravenous fluorouracil and leucovorin in stage II and III carcinoma of the colon: results from National Surgical Adjuvant Breast and Bowel Project Protocol C-06. J Clin Oncol 24:2059-2064

Publisher's Note Springer Nature remains neutral with regard to jurisdictional claims in published maps and institutional affiliations. 


\section{Affiliations}

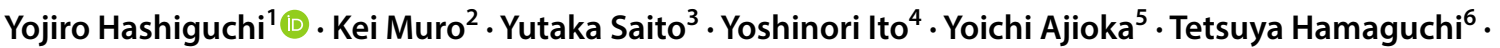
Kiyoshi Hasegawa $^{7} \cdot$ Kinichi Hotta $^{8} \cdot$ Hideyuki Ishida $^{9} \cdot$ Megumi Ishiguro $^{10} \cdot$ Soichiro Ishihara ${ }^{11}$. Yukihide Kanemitsu ${ }^{12}$. Yusuke Kinugasa ${ }^{13} \cdot$ Keiko Murofushi $^{14}$. Takako Eguchi Nakajima ${ }^{15}$. Shiro Oka ${ }^{16}$. Toshiaki Tanaka $^{11}$. Hiroya Taniguchi ${ }^{17}$. Akihito Tsuji ${ }^{18} \cdot K^{1}$ eisuke Uehara ${ }^{19} \cdot$ Hideki Ueno $^{20} \cdot$ Takeharu Yamanaka $^{21}$. Kentaro Yamazaki ${ }^{22} \cdot$ Masahiro Yoshida $^{23}$. Takayuki Yoshino ${ }^{17} \cdot$ Michio Itabashi $^{24} \cdot$ Kentaro Sakamaki $^{25} \cdot$ Keiji Sano $^{1}$. Yasuhiro Shimada ${ }^{26}$. Shinji Tanaka ${ }^{27} \cdot$ Hiroyuki Uetake $^{28} \cdot$ Shigeki Yamaguchi $^{29} \cdot$ Naohiko Yamaguchi $^{30}$. Hirotoshi Kobayashi $^{31} \cdot$ Keiji Matsuda $^{1} \cdot K_{\text {Kenjiro Kotake }}^{32} \cdot$ Kenichi Sugihara $^{33}$. Japanese Society for Cancer of the Colon and Rectum

1 Department of Surgery, Teikyo University School of Medicine, 2-11-1 Kaga, Itabashi-ku, Tokyo 173-8606, Japan

2 Department of Clinical Oncology, Aichi Cancer Center Hospital, Nagoya, Japan

3 Endoscopy Division, National Cancer Center Hospital, Tokyo, Japan

4 Department of Radiation Oncology, Showa University School of Medicine, Tokyo, Japan

5 Division of Molecular and Diagnostic Pathology, Graduate School of Medical and Dental Sciences, Niigata University, Niigata, Japan

6 Department of Gastroenterological Oncology, Saitama Medical University International Medical Center, Saitama, Japan

7 Hepato-Biliary-Pancreatic Surgery Division, Artificial Organ and Transplantation Division, Department of Surgery, Graduate School of Medicine, The University of Tokyo, Tokyo, Japan

8 Division of Endoscopy, Shizuoka Cancer Center, Shizuoka, Japan

9 Department of Digestive Tract and General Surgery, Saitama Medical Center, Saitama Medical University, Saitama, Japan

10 Department of Chemotherapy and Oncosurgery, Tokyo Medical and Dental University Medical Hospital, Tokyo, Japan

11 Department of Surgical Oncology, Graduate School of Medicine, The University of Tokyo, Tokyo, Japan

12 Department of Colorectal Surgery, National Cancer Center Hospital, Tokyo, Japan

13 Department of Gastrointestinal Surgery, Tokyo Medical and Dental University, Tokyo, Japan

14 Department of Radiation Oncology, faculty of Medicine, University of Tsukuba, Ibaraki, Japan

15 Department of Clinical Oncology, St. Marianna University School of Medicine, Kawasaki, Japan

16 Department of Gastroenterology and Metabolism, Hiroshima University Hospital, Hiroshima, Japan
17 Department of Gastroenterology and Gastrointestinal Oncology, National Cancer Center Hospital East, Chiba, Japan

18 Department of Clinical Oncology, Faculty of Medicine, Kagawa University, Kagawa, Japan

19 Division of Surgical Oncology, Department of Surgery, Nagoya University Graduate School of Medicine, Aichi, Japan

20 Department of Surgery, National Defense Medical College, Saitama, Japan

21 Department of Biostatistics, Yokohama City University School of Medicine, Yokohama, Japan

22 Division of Gastrointestinal Oncology, Shizuoka Cancer Center, Shizuoka, Japan

23 Department of Hepato-Biliary-Pancreatic and Gastrointestinal Surgery, School of Medicine, International University of Health and Welfare, Narita, Japan

24 Department of Surgery, Institute of Gastroenterology, Tokyo Women's Medical University, Tokyo, Japan

25 Center for Data Science, Yokohama City University, Yokohama, Japan

26 Division of Clinical Oncology, Kochi Health Sciences Center, Kochi, Japan

27 Department of Endoscopy, Hiroshima University Hospital, Hiroshima, Japan

28 Department of Specialized Surgeries, Tokyo Medical and Dental University, Tokyo, Japan

29 Department of Gastroenterological Surgery, Saitama Medical University International Medical Center, Hidaka, Japan

30 Library of SEIREI, SAKURA Citizen Hospital, Sakura, Japan

31 Department of Surgery, Mizonokuchi Hospital, Teikyo University School of Medicine, Kanagawa, Japan

32 Department of Surgery, Sano City Hospital, Tochigi, Japan

33 Tokyo Medical and Dental University, Tokyo, Japan 